\title{
STATISTICS FOR LOW-LYING ZEROS OF SYMMETRIC POWER $L$-FUNCTIONS IN THE LEVEL ASPECT
}

\author{
GUILLAUME RICOTTA AND EMMANUEL ROYER
}

\begin{abstract}
We study one-level and two-level densities for low lying zeros of symmetric power $L$-functions in the level aspect. It allows us to completely determine the symmetry types of some families of symmetric power $L$-functions with prescribed sign of functional equation. We also compute the moments of one-level density and exhibit mock-Gaussian behavior discovered by Hughes \& Rudnick.
\end{abstract}

\section{COntents}

1. Introduction and statement of the results 2

1.1. Description of the families of $L$-functions studied 2

1.2. Symmetry type of these families 3

1.2.1. (Signed) asymptotic expectation of the one-level density 4

1.2.2. Sketch of the proof 5

1.2.3. (Signed) asymptotic expectation of the two-level density 7

1.3. Asymptotic moments of the one-level density 8

1.4. Organisation of the paper 10

2. Automorphic and probabilistic background 10

2.1. Automorphic background 10

2.1.1. Overview of holomorphic cusp forms 10

2.1.2. Chebyshev polynomials and Hecke eigenvalues 13

2.1.3. Overview of $L$-functions associated to primitive cusp forms 13

2.1.4. Overview of symmetric power $L$-functions 14

2.2. Probabilistic background 15

3. Main technical ingredients of this work 17

3.1. Large sieve inequalities for Kloosterman sums 17

3.2. Riemann's explicit formula for symmetric power $L$-functions $\quad 19$

3.3. Contribution of the old forms 21

4. Linear statistics for low-lying zeros 23

4.1. Density results for families of $L$-functions 23

4.2. Asymptotic expectation of the one-level density 24

4.3. Signed asymptotic expectation of the one-level density 27

5. Quadratic statistics for low-lying zeros 29

5.1. Asymptotic expectation of the two-level density and asymptotic variance

5.2. Signed asymptotic expectation of the two-level density and signed asymptotic variance 31

6. First asymptotic moments of the one-level density 33

Date: Version of March 26, 2007. 
6.1. One some useful combinatorial identity 35

6.2. Proof of the first bullet of proposition 6.1

6.3. Proof of the third bullet of proposition $6.1 \quad 37$

6.4. Proof of the second bullet of proposition $6.1 \quad 40$

Appendix A. Analytic and arithmetic toolbox 44

A.1. On smooth dyadic partitions of unity 44

A.2. On Bessel functions $\quad 45$

A.3. Basic facts on Kloosterman sums 46

References $\quad 46$

Acknowledgements- This work began while both authors shared the hospitality of Centre de Recherches Mathématiques (Montréal) during the theme year "Analysis in Number Theory" (first semester of 2006). We would like to thank C. David, H. Darmon and A. Granville for their invitation. This work was essentially completed in september 2006 in CIRM (Luminy) at the occasion of J.-M. Deshouillers' sixtieth birthday. We would like to wish him the best.

\section{IntRoduction AND STATEMENT OF THE RESUlts}

1.1. Description of the families of $L$-functions studied. The purpose of this paper is to compute various statistics associated to low-lying zeros of several families of symmetric power $L$-functions in the level aspect. First of all, we give a short description of these families. To any primitive holomorphic cusp form $f$ of prime level $q$ and even weight ${ }^{1} \kappa \geqslant 2$ (see $\S 2.1$ for the automorphic background) say $f \in H_{\kappa}^{*}(q)$, one can associate its $r$-th symmetric power $L$-function denoted by $L\left(\operatorname{Sym}^{r} f, s\right)$ for any integer $r \geqslant 1$. It is given by an explicit absolutely convergent Euler product of degree $r+1$ on $\Re e s>1$ (see $\S 2.1 .4$ ). The completed $L$-function is defined by

$$
\Lambda\left(\operatorname{Sym}^{r} f, s\right):=\left(q^{r}\right)^{s / 2} L_{\infty}\left(\operatorname{Sym}^{r} f, s\right) L\left(\operatorname{Sym}^{r} f, s\right)
$$

where $L_{\infty}\left(\operatorname{Sym}^{r} f, s\right)$ is a product of $r+1$ explicit $\Gamma_{\mathbb{R}}$-factors (see $\left.\S 2.1 .4\right)$ and $q^{r}$ is the arithmetic conductor. We will need some control on the analytic behaviour of this function. Unfortunately, such information is not currently known in all generality. We sum up our main assumption in the following statement.

Hypothesis Nice $(r, f)$ - The function $\Lambda\left(\operatorname{Sym}^{r} f, s\right)$ is a completed $L$-function in the sense that it satisfies the following nice analytic properties:

- it can be extended to an holomorphic function of order 1 on $\mathbb{C}$,

- it satisfies a functional equation of the shape

$$
\Lambda\left(\operatorname{Sym}^{r} f, s\right)=\varepsilon\left(\operatorname{Sym}^{r} f\right) \Lambda\left(\operatorname{Sym}^{r} f, 1-s\right)
$$

\footnotetext{
${ }^{1}$ In this paper, the weight $\kappa$ is a fixed even integer and the level $q$ goes to infinity among the prime numbers.
} 
where the sign $\varepsilon\left(\operatorname{Sym}^{r} f\right)= \pm 1$ of the functional equation is given by

$$
\varepsilon\left(\operatorname{Sym}^{r} f\right):= \begin{cases}+1 & \text { if } r \text { is even } \\ \varepsilon_{f}(q) \times \varepsilon(\kappa, r) & \text { otherwise }\end{cases}
$$

with

$$
\varepsilon(\kappa, r):=i^{\left(\frac{r+1}{2}\right)^{2}(\kappa-1)+\frac{r+1}{2}}=\left\{\begin{array}{lll}
i^{\kappa} & \text { if } r \equiv 1 & (\bmod 8), \\
-1 & \text { if } r \equiv 3 & (\bmod 8), \\
-i^{\kappa} & \text { if } r \equiv 5 & (\bmod 8), \\
+1 & \text { if } r \equiv 7 & (\bmod 8)
\end{array}\right.
$$

and $\varepsilon_{f}(q)= \pm 1$ is defined in (2.15) and only depends on $f$ and $q$.

Remark 1- Hypothesis Nice $(r, f)$ is known for $r=1$ (E. Hecke [10-12]), $r=2$ thanks to the work of S. Gelbart and H. Jacquet [8] and $r=3,4$ from the works of H. Kim and F. Shahidi [20-22]. by

We aim at studying the low-lying zeros for the family of $L$-functions given

$$
\mathcal{F}_{r}:=\bigcup_{q \text { prime }}\left\{L\left(\operatorname{Sym}^{r} f, s\right), f \in H_{\kappa}^{*}(q)\right\}
$$

for any integer $r \geqslant 1$. Note that when $r$ is even, the sign of the functional equation of any $L\left(\operatorname{Sym}^{r} f, s\right)$ is constant of value +1 but when $r$ is odd, this is definitely not the case. As a consequence, it is very natural to understand the low-lying zeros for the subfamilies given by

$$
\mathcal{F}_{r}^{\varepsilon}:=\bigcup_{q \text { prime }}\left\{L\left(\operatorname{Sym}^{r} f, s\right), f \in H_{\kappa}^{*}(q), \varepsilon\left(\operatorname{Sym}^{r} f\right)=\varepsilon\right\}
$$

for any odd integer $r \geqslant 1$ and for $\varepsilon= \pm 1$.

1.2. Symmetry type of these families. One of the purpose of this work is to determine the symmetry type of the families $\mathcal{F}_{r}$ and $\mathcal{F}_{r}^{\varepsilon}$ for $\varepsilon= \pm 1$ and for any integer $r \geqslant 1$ (see $\S 4.1$ for the background on symmetry types). The following theorem is a quick summary of the symmetry types obtained.

Theorem $\mathbf{A}-$ Let $r \geqslant 1$ be any integer and $\varepsilon= \pm 1$. We assume that hypothesis Nice $(r, f)$ holds for any prime number $q$ and any primitive holomorphic cusp form of level $q$ and even weight $\kappa \geqslant 2$. The symmetry group $G\left(\mathcal{F}_{r}\right)$ of $\mathcal{F}_{r}$ is given by

$$
G\left(\mathcal{F}_{r}\right)= \begin{cases}S p & \text { if } r \text { is even, } \\ O & \text { otherwise. }\end{cases}
$$

If $r$ is odd then the symmetry group $G\left(\mathcal{F}_{r}^{\varepsilon}\right)$ of $\mathcal{F}_{r}^{\varepsilon}$ is given by

$$
G\left(\mathcal{F}_{r}^{\varepsilon}\right)= \begin{cases}S O(\text { even }) & \text { if } \varepsilon=+1 \\ S O(\text { odd }) & \text { otherwise. }\end{cases}
$$

Remark 2- It follows in particular from the value of $\varepsilon\left(\mathrm{Sym}^{r} f\right)$ given in (1.1) that, if $r$ is even, then $\operatorname{Sym}^{r} f$ has not the same symmetry type than $f$ and, if $r$ is odd, then $f$ and $\operatorname{Sym}^{r} f$ have the same symmetry type if and only if

$$
r \equiv 1 \quad(\bmod 8) \text { and } \kappa \equiv 0 \quad(\bmod 4)
$$


or

$$
r \equiv 5 \quad(\bmod 8) \text { and } \kappa \equiv 2 \quad(\bmod 4)
$$

or

$$
r \equiv 7 \quad(\bmod 8)
$$

Remark 3- Note that we do not assume any Generalised Riemann Hypothesis for the symmetric power $L$-functions.

In order to prove theorem A, we compute either the (signed) asymptotic expectation of the one-level density or the (signed) asymptotic expectation of the two-level density. The results are given in the next two sections in which $\varepsilon= \pm 1, \nu$ will always be a positive real number, $\Phi, \Phi_{1}$ and $\Phi_{2}$ will always stand for even Schwartz functions whose Fourier transforms $\widehat{\Phi}, \widehat{\Phi_{1}}$ and $\widehat{\Phi_{2}}$ are compactly supported in $[-\nu,+\nu]$ and $f$ will always be a primitive holomorphic cusp form of prime level $q$ and even weight $\kappa \geqslant 2$ for which hypothesis Nice $(r, f)$ holds. We refer to $\S 2.2$ for the probabilistic background.

1.2.1. (Signed) asymptotic expectation of the one-level density. The one-level density (relatively to $\Phi$ ) of $\operatorname{Sym}^{r} f$ is defined by

$$
D_{1, q}[\Phi ; r](f):=\sum_{\rho, \Lambda\left(\mathrm{Sym}^{r} f, \rho\right)=0} \Phi\left(\frac{\log \left(q^{r}\right)}{2 i \pi}\left(\Re e \rho-\frac{1}{2}+i \Im m \rho\right)\right)
$$

where the sum is over the non-trivial zeros $\rho$ of $L\left(\operatorname{Sym}^{r} f, s\right)$ with multiplicities. The asymptotic expectation of the one-level density is by definition

$$
\lim _{\substack{q \text { prime } \\ q \rightarrow+\infty}}[r] \sum_{f \in H_{\kappa}^{*}(q)} \omega_{q}(f) D_{1, q}[\Phi ; r](f)
$$

where $\omega_{q}(f)$ is the harmonic weight defined in (2.7) and similarly the signed asymptotic expectation of the one-level density is by definition

$$
\lim _{\substack{q \text { prime } \\ q \rightarrow+\infty}} 2[r] \sum_{\substack{f \in H_{\kappa}^{*}(q) \\ \varepsilon\left(\operatorname{Sym}^{r} f\right)=\varepsilon}} \omega_{q}(f) D_{1, q}[\Phi ; r](f)
$$

when $r$ is odd.

Theorem $\mathbf{B}-$ Let $r \geqslant 1$ be any integer and $\varepsilon= \pm 1$. We assume that hypothesis $\operatorname{Nice}(r, f)$ holds for any prime number $q$ and any primitive holomorphic cusp form of level $q$ and even weight $\kappa \geqslant 2$ and also that $\theta$ is admissible (see hypothesis $\mathrm{H}_{2}(\theta)$ page 17). Let

$$
\nu_{1, \max }(r, \kappa, \theta):=\left(1-\frac{1}{2(\kappa-2 \theta)}\right) \frac{2}{r^{2}} .
$$

If $\nu<\nu_{1, \max }(r, \kappa, \theta)$ then the asymptotic expectation of the one-level density is

Let

$$
\widehat{\Phi}(0)+\frac{(-1)^{r+1}}{2} \Phi(0) .
$$

$$
\nu_{1, \max }^{\varepsilon}(r, \kappa, \theta):=\inf \left(\nu_{1, \max }(r, \kappa, \theta), \frac{3}{r(r+2)}\right) .
$$


If $r$ is odd and $\nu<\nu_{1, \max }^{\varepsilon}(r, \kappa, \theta)$ then the signed asymptotic expectation of the one-level density is

$$
\widehat{\Phi}(0)+\frac{(-1)^{r+1}}{2} \Phi(0) .
$$

Remark 4- The first part of Theorem B reveals that the symmetry type of $\mathcal{F}_{r}$ is

$$
G\left(\mathcal{F}_{r}\right)= \begin{cases}S p & \text { if } r \text { is even, } \\ O & \text { if } r=1, \\ S O(\text { even }) \text { or } O \text { or } S O(\text { odd }) & \text { if } r \geqslant 3 \text { is odd. }\end{cases}
$$

We cannot decide between the three orthogonal groups when $r \geqslant 3$ is odd since in this case $\nu_{1, \max }(r, \kappa, \theta)<1$ but the computation of the two-level densities will enable us to decide. Note also that we go beyond the support $[-1,1]$ when $r=1$ as Iwaniec, Luo \& Sarnak [18] (Theorem 1.1) but without doing any subtle arithmetic analysis of Kloosterman sums. Also, A. Güloglu in [9, Theorem 1.2] established some density result for the same family of $L$-functions but when the weight $\kappa$ goes to infinity and the level $q$ is fixed. It turns out that we recover the same constraint on $\nu$ when $r$ is even but we get a better result when $r$ is odd. This can be explained by the fact that the analytic conductor of any $L\left(\operatorname{Sym}^{r} f, s\right)$ with $f$ in $H_{\kappa}^{*}(q)$ which is of size

$$
q^{r} \times \begin{cases}\kappa^{r} & \text { if } r \text { is even } \\ \kappa^{r+1} & \text { otherwise }\end{cases}
$$

is slightly larger in his case than in ours when $r$ is odd.

Remark 5- The second part of Theorem B reveals that if $r$ is odd and $\varepsilon= \pm 1$ then the symmetry type of $\mathcal{F}_{r}^{\varepsilon}$ is

$$
G\left(\mathcal{F}_{r}^{\varepsilon}\right)=S O(\text { even }) \text { or } O \text { or } S O(\text { odd }) .
$$

Here $\nu$ is always strictly smaller than one and we are not able to recover the result of [18, Theorem 1.1] without doing some arithmetic on Kloosterman sums.

1.2.2. Sketch of the proof. We give here a sketch of the proof of the first part of Theorem B namely we briefly explain how to determine the asymptotic expectation of the one-level density assuming that hypothesis $\operatorname{Nice}(r, f)$ holds for any prime number $q$ and any primitive holomorphic cusp form of level $q$ and even weight $\kappa \geqslant 2$ and also that $\theta$ is admissible. The first step consists in transforming the sum over the zeros of $\Lambda\left(\mathrm{Sym}^{r} f, s\right)$ which occurs in $D_{1, q}[\Phi ; r](f)$ into a sum over primes. This is done via some Riemann's explicit formula for symmetric power $L$-functions stated in Proposition 3.8 which leads to

$$
D_{1, q}[\Phi ; r](f)=\widehat{\Phi}(0)+\frac{(-1)^{r+1}}{2} \Phi(0)+P_{q}^{1}[\Phi ; r](f)+\sum_{m=0}^{r-1}(-1)^{m} P_{q}^{2}[\Phi ; r, m](f)+o(1)
$$

where

$$
P_{q}^{1}[\Phi ; r](f):=-\frac{2}{\log \left(q^{r}\right)} \sum_{\substack{p \in \mathcal{P} \\ p \nmid q}} \lambda_{f}\left(p^{r}\right) \frac{\log p}{\sqrt{p}} \widehat{\Phi}\left(\frac{\log p}{\log \left(q^{r}\right)}\right)
$$


The terms $P_{q}^{2}[\Phi ; r, m](f)$ are also sums over primes which look like $P_{q}^{1}[\Phi ; r](f)$ but can be forgotten in first approximation since they can be thought as sums over squares of primes which are easier to deal with. The second step consists in averaging over all the $f$ in $H_{\kappa}^{*}(q)$. While doing this, the asymptotic expectation of the one-level density

$$
\widehat{\Phi}(0)+\frac{(-1)^{r+1}}{2} \Phi(0)
$$

naturally appears and we need to show that

$$
-\frac{2}{\log \left(q^{r}\right)} \sum_{\substack{p \in \mathcal{P} \\ p \nmid q}}\left(\sum_{f \in H_{\kappa}^{*}(q)} \omega_{q}(f) \lambda_{f}\left(p^{r}\right)\right) \frac{\log p}{\sqrt{p}} \widehat{\Phi}\left(\frac{\log p}{\log \left(q^{r}\right)}\right)
$$

is a remainder term provided that the support $\nu$ of $\Phi$ is small enough. We apply some suitable trace formula given in Proposition 2.2 in order to express the previous average of Hecke eigenvalues. We cannot directly apply Peterson's trace formula since there may be some old forms of level $q$ especially when the weight $\kappa$ is large. Nevertheless, these old forms are automatically of level 1 since $q$ is prime and their contribution remains negligible. So, we have to bound

$$
-\frac{4 \pi i^{\kappa}}{\log \left(q^{r}\right)} \sum_{\substack{p \in \mathcal{P} \\ p \nmid q}} \sum_{\substack{q \geqslant 1 \\ q \mid c}} \frac{S\left(1, p^{r} ; c\right)}{c} J_{\kappa-1}\left(\frac{4 \pi \sqrt{p^{r}}}{c}\right) \frac{\log p}{\sqrt{p}} \widehat{\Phi}\left(\frac{\log p}{\log \left(q^{r}\right)}\right)
$$

where $S\left(1, p^{r} ; c\right)$ is a Kloosterman sum and which can be written as

$$
-\frac{4 \pi i^{\kappa}}{\log \left(q^{r}\right)} \sum_{\substack{c \geqslant 1 \\ q \mid c}} \sum_{m \geqslant 1} a_{m} \frac{S(1, m ; c)}{c} g(m ; c)
$$

where

$$
a_{m}:=1_{\left[1, q^{r^{2} \nu}\right]}(m) \frac{\log m}{r m^{1 /(2 r)}} \times \begin{cases}1 & \text { if } m=p^{r} \text { for some prime } p \neq q, \\ 0 & \text { otherwise }\end{cases}
$$

and

$$
g(m ; c):=J_{\kappa-1}\left(\frac{4 \pi \sqrt{m}}{c}\right) \widehat{\Phi}\left(\frac{\log m}{r \log \left(q^{r}\right)}\right) .
$$

We apply the large sieve inequality for Kloosterman sums given in proposition 3.4. It entails that if $\nu \leqslant 2 / r^{2}$ then such quantity is bounded by

$$
\ll_{\varepsilon} q^{\left(\frac{\kappa-1}{2}-\theta\right)\left(r^{2} \nu-2\right)+\varepsilon}+q^{\left(\frac{\kappa}{2}-\theta\right) r^{2} \nu-\left(\kappa-\frac{1}{2}-2 \theta\right)+\varepsilon} .
$$

This is an admissible error term if $\nu<\nu_{1, \max }(r, \kappa, \theta)$. We focus on the fact that we did any arithmetic analysis of Kloosterman sums to get this result. Of course, the power of spectral theory of automorphic forms is hidden in the large sieve inequalities for Kloosterman sums. 
1.2.3. (Signed) asymptotic expectation of the two-level density. The two-level density of $\operatorname{Sym}^{r} f$ (relatively to $\Phi_{1}$ and $\Phi_{2}$ ) is defined by

$$
D_{2, q}\left[\Phi_{1}, \Phi_{2} ; r\right](f):=\sum_{\substack{\left(j_{1}, j_{2}\right) \in \mathcal{E}(f, r)^{2} \\ j_{1} \neq \pm j_{2}}} \Phi_{1}\left(\widehat{\rho}_{f, r}^{\left(j_{1}\right)}\right) \Phi_{2}\left(\widehat{\rho}_{f, r}^{\left(j_{2}\right)}\right) .
$$

For more precision on the numbering of the zeros, we refer to $\S 3.2$. The asymptotic expectation of the two-level density is by definition

$$
\lim _{\substack{q \\ q \rightarrow+\infty}}[r] \sum_{f \in H_{\kappa}^{*}(q)} \omega_{q}(f) D_{2, q}\left[\Phi_{1}, \Phi_{2} ; r\right](f)
$$

and similarly the signed asymptotic expectation of the two-level density is by definition

$$
\lim _{\substack{q \text { prime } \\ q \rightarrow+\infty}} 2[r] \sum_{\substack{f \in H_{\kappa}^{*}(q) \\ \varepsilon\left(\operatorname{Sym}^{r} f\right)=\varepsilon}} \omega_{q}(f) D_{2, q}\left[\Phi_{1}, \Phi_{2} ; r\right](f)
$$

when $r$ is odd and $\varepsilon= \pm 1$.

Theorem $\mathbf{C}-$ Let $r \geqslant 1$ be any integer and $\varepsilon= \pm 1$. We assume that hypothesis $\operatorname{Nice}(r, f)$ holds for any prime number $q$ and any primitive holomorphic cusp form of level $q$ and even weight $\kappa \geqslant 2$. If $\nu<1 / r^{2}$ then the asymptotic expectation of the two-level density is

$$
\begin{aligned}
& {\left[\widehat{\Phi_{1}}(0)+\frac{(-1)^{r+1}}{2} \Phi_{1}(0)\right]\left[\widehat{\Phi_{2}}(0)+\frac{(-1)^{r+1}}{2} \Phi_{2}(0)\right]} \\
& +2 \int_{\mathbb{R}}|u| \widehat{\Phi_{1}}(u) \widehat{\Phi_{2}}(u) \mathrm{d} u-2 \widehat{\Phi_{1} \Phi_{2}}(0)+\left((-1)^{r}+\frac{1_{2 \mathbb{N}+1}(r)}{2}\right) \Phi_{1}(0) \Phi_{2}(0) .
\end{aligned}
$$

If $r$ is odd and $\nu<1 /(2 r(r+2))$ then the signed asymptotic expectation of the two-level density is

$$
\begin{aligned}
{\left[\widehat{\Phi_{1}}(0)+\right.} & \left.\frac{1}{2} \Phi_{1}(0)\right]\left[\widehat{\Phi_{2}}(0)+\frac{1}{2} \Phi_{2}(0)\right] \\
& +2 \int_{\mathbb{R}}|u| \widehat{\Phi_{1}}(u) \widehat{\Phi_{2}}(u) \mathrm{d} u-2 \widehat{\Phi_{1} \Phi_{2}}(0)-\Phi_{1}(0) \Phi_{2}(0) \\
& +1_{\{-1\}}(\varepsilon) \Phi_{1}(0) \Phi_{2}(0) .
\end{aligned}
$$

Remark 6- We have just seen that the computation of the one-level density already reveals that the symmetry type of $\mathcal{F}_{r}$ is $S p$ when $r$ is even. The asymptotic expectation of the two-level density also coincides with the one of $S p$ (see [19, Theorem A.D.2.2] or [26, Theorem 3.3]). When $r \geqslant 3$ is odd, the first part of Theorem C together with a result of Katz \& Sarnak (see [19, Theorem A.D.2.2] or [26, Theorem 3.2]) imply that the symmetry type of $\mathcal{F}_{r}$ is $O$.

Remark 7- The second part of Theorem C and a result of Katz \& Sarnak (see [19, Theorem A.D.2.2] or [26, Theorem 3.2]) imply that the symmetry type of $\mathcal{F}_{r}^{\varepsilon}$ is as in Theorem A for any odd integer $r \geqslant 1$ and $\varepsilon= \pm 1$. 
In order to prove Theorem $\mathrm{C}$, we need to determine the asymptotic variance of the one-level density which is defined by

$$
\lim _{\substack{q \text { prime } \\ q \rightarrow+\infty}}[r] \sum_{f \in H_{\kappa}^{*}(q)} \omega_{q}(f)\left(D_{1, q}[\Phi ; r](f)-\sum_{g \in H_{\kappa}^{*}(q)} \omega_{q}(g) D_{1, q}[\Phi ; r](g)\right)^{2}
$$

and the signed asymptotic variance of the one-level density which is similarly defined by

$\lim _{\substack{q \text { prime } \\ q \rightarrow+\infty}} 2[r] \sum_{\substack{f \in H_{r}^{*}(q) \\ \varepsilon\left(\operatorname{Sym}^{r} f\right)=\varepsilon}} \omega_{q}(f)\left(D_{1, q}[\Phi ; r](f)-2[r] \sum_{\substack{g \in H_{r}^{*}(q) \\ \varepsilon\left(\operatorname{Sym}^{r} g\right)=\varepsilon}} \omega_{q}(g) D_{1, q}[\Phi ; r](g)\right)^{2}$

when $r$ is odd and $\varepsilon= \pm 1$.

Theorem $\mathbf{D}-$ Let $r \geqslant 1$ be any integer and $\varepsilon= \pm 1$. We assume that hypothesis $\operatorname{Nice}(r, f)$ holds for any prime number $q$ and any primitive holomorphic cusp form of level $q$ and even weight $\kappa \geqslant 2$. If $\nu<1 / r^{2}$ then the asymptotic variance of the one-level density is

$$
2 \int_{\mathbb{R}}|u| \widehat{\Phi}^{2}(u) \mathrm{d} u
$$

If $r$ is odd and $\nu<1 /(2 r(r+2))$ then the signed asymptotic variance of the one-level density is

$$
2 \int_{\mathbb{R}}|u| \widehat{\Phi}^{2}(u) \mathrm{d} u .
$$

1.3. Asymptotic moments of the one-level density. Last but not least, we compute the asymptotic m-th moment of the one-level density which is defined by

$$
\lim _{\substack{q \text { prime } \\ q \rightarrow+\infty}}[r] \sum_{f \in H_{\kappa}^{*}(q)} \omega_{q}(f)\left(D_{1, q}[\Phi ; r](f)-\sum_{g \in H_{\kappa}^{*}(q)} \omega_{q}(g) D_{1, q}[\Phi ; r](g)\right)^{m}
$$

for any integer $m \geqslant 1$.

Theorem $\mathbf{E}-$ Let $r \geqslant 1$ be any integer and $\varepsilon= \pm 1$. We assume that hypothesis $\operatorname{Nice}(r, f)$ holds for any prime number $q$ and any primitive holomorphic cusp form of level $q$ and even weight $\kappa \geqslant 2$. If $m \nu<4 /(r(r+2))$ then the asymptotic m-th moment of the one-level density is

$$
\begin{cases}0 & \text { if } m \text { is odd, } \\ 2 \int_{\mathbb{R}}|u| \widehat{\Phi}^{2}(u) \mathrm{d} u \times \frac{m !}{2^{m / 2}\left(\frac{m}{2}\right) !} & \text { otherwise. }\end{cases}
$$

Remark 8- This result is another evidence for mock-Gaussian behaviour (see [13-15] for instance).

Remark 9- We compute the first asymptotic moments of the one-level density. These computations allow to compute the asymptotic expectation of the first level-densities $[13, \S 1.2]$. We will use the specific case of the asymptotic expectation of the two-level density and the asymptotic variance in $\S 5.1$. 
Let us sketch the proof of Theorem E by explaining the origin of the main term. We have to evaluate

$$
\sum_{\substack{0 \leqslant \ell \leqslant m \\
0 \leqslant \alpha \leqslant \ell}}\left(\begin{array}{c}
m \\
\ell
\end{array}\right)\left(\begin{array}{c}
\ell \\
\alpha
\end{array}\right) R(q)^{\ell-\alpha} \mathbb{E}_{q}^{\mathrm{h}}\left(P_{q}^{1}[\Phi ; r]^{m-\ell} P_{q}^{2}[\Phi ; r]^{\alpha}\right)
$$

where $P_{q}^{1}[\Phi ; r]$ has been defined in (1.2),

$$
P_{q}^{2}[\Phi ; r](f)=-\frac{2}{\log \left(q^{r}\right)} \sum_{j=1}^{r}(-1)^{r-j} \sum_{\substack{p \in \mathcal{P} \\ p \nmid q}} \lambda_{f}\left(p^{2 j}\right) \frac{\log p}{p} \widehat{\Phi}\left(\frac{2 \log p}{\log \left(q^{r}\right)}\right)
$$

and $R(q)$ satisfies

$$
R(q)=O\left(\frac{1}{\log q}\right) .
$$

The main term comes from the contribution $\ell=0$ in the sum (1.3). Using a combinatorial lemma, we rewrite this main contribution as

$$
\frac{(-2)^{m}}{\log ^{m}\left(q^{r}\right)} \sum_{s=1}^{m} \sum_{\sigma \in P(m, s)} \sum_{\begin{array}{c}
i_{1}, \ldots, i_{s} \\
\text { distinct }
\end{array}} \mathbb{E}_{q}^{\mathrm{h}}\left(\prod_{u=1}^{s} \lambda_{f}\left(\widehat{p}_{i_{u}}^{r}\right)^{\varpi_{u}^{(\sigma)}}\right)
$$

where $P(m, s)$ is the set of surjective functions

$$
\sigma:\{1, \ldots, \alpha\} \rightarrow\{1, \ldots, s\}
$$

such that for any $j \in\{1, \ldots, s\}$, either $\sigma(j)=1$ or there exists $k<j$ such that $\sigma(j)=\sigma(k)+1$ and for any $j \in\{1, \ldots, s\}$

$$
\varpi_{j}^{(\sigma)}:=\# \sigma^{-1}(\{j\}) .
$$

$\left(\widehat{p}_{i}\right)_{i \geqslant 1}$ stands for the increasing sequence of prime numbers different from $q$. Linearising each $\lambda_{f}\left(\widehat{p}_{i_{u}}^{r}\right)^{\varpi_{u}^{(\sigma)}}$ in terms of $\lambda_{f}\left(\hat{p}_{i_{u}}^{j_{u}}\right)$ with $j_{u}$ runs over integers in $\left[0, r \varpi_{u}^{(\sigma)}\right]$ and using a trace formula to prove that the only $\sigma \in P(m, s)$ leading to a principal contribution satisfy $\varpi_{j}^{(\sigma)}=2$ for any $j \in\{1, \ldots, s\}$, we have to estimate

$$
\frac{(-2)^{m}}{\log ^{m}\left(q^{r}\right)} \sum_{s=1}^{m} \sum_{\substack{\sigma \in P(m, s) \\ \forall j \in\{1, \ldots, s\}, \varpi_{j}^{(\sigma)}=2}} \sum_{\substack{i_{1}, \ldots, i_{s} \\ \text { distinct }}} \prod_{u=1}^{s} \frac{\log ^{2}\left(\widehat{p}_{i_{u}}\right)}{\widehat{p}_{i_{u}}} \widehat{\Phi}^{2}\left(\frac{\log \widehat{p}_{i_{u}}}{\log \left(q^{r}\right)}\right) .
$$

This sum vanishes if $m$ is odd since

$$
\sum_{j=1}^{s} \varpi_{j}^{(\sigma)}=m
$$

and it remains to prove the formula for $m$ even. In this case, and since we already computed the moment for $m=2$, we deduce from (1.4) that the main contribution is

$$
\mathbb{E}_{q}^{\mathrm{h}}\left(P_{q}^{1}[\Phi ; r]^{2}\right) \times \#\left\{\sigma \in P(m, m / 2): \varpi_{j}^{(\sigma)}=2(\forall j)\right\}
$$


and we conclude by computing

$$
\#\left\{\sigma \in P(m, m / 2): \varpi_{j}^{(\sigma)}=2(\forall j)\right\}=\frac{m !}{2^{m / 2}\left(\frac{m}{2}\right) !} .
$$

Proving that the other terms lead to error terms is done by implementing similar ideas, but requires - especially for the double products (namely terms implying both $P_{q}^{1}$ and $P_{q}^{2}$ ) - much more combinatorial technicalities.

1.4. Organisation of the paper. Section 2 contains the automorphic and probabilistic background which is needed to be able to read this paper. In particular, we give here the accurate definition of symmetric power $L$ functions and the properties of Chebyshev polynomials useful in section 6 . In section 3, we describe the main technical ingredients of this work namely large sieve inequalities for Kloosterman sums and Riemann's explicit formula for symmetric power $L$-functions. In section 4 , some standard facts about symmetry groups are given and the computation of the (signed) asymptotic expectation of the one-level density is done. The computations of the (signed) asymptotic expectation, covariance and variance of the twolevel density are done in section 5 whereas the computation of the asymptotic moments of the one-level density is provided in section 6. Some well-known facts about Kloosterman sums are recalled in appendix A.

Notation- We write $\mathcal{P}$ for the set of prime numbers and the main parameter in this paper is a prime number $q$, whose name is the level, which goes to infinity among $\mathcal{P}$. Thus, if $f$ and $g$ are some $\mathbb{C}$-valued functions of the real variable then the notations $f(q) \ll_{A} g(q)$ or $f(q)=O_{A}(g(q))$ mean that $|f(q)|$ is smaller than a "constant" which only depends on A times $g(q)$ at least for $q$ a large enough prime number and similarly, $f(q)=o(1)$ means that $f(q) \rightarrow 0$ as $q$ goes to infinity among the prime numbers. We will denote by $\varepsilon$ an absolute positive constant whose definition may vary from one line to the next one. The characteristic function of a set $S$ will be denoted $1_{S}$.

\section{Automorphic AND PRobabilistic BaCKGround}

\subsection{Automorphic background.}

2.1.1. Overview of holomorphic cusp forms. In this section, we recall general facts about holomorphic cusp forms. A reference is [16].

Generalities - We write $\Gamma_{0}(q)$ for the congruence subgroup of level $q$ which acts on the upper-half plane $\mathcal{H}$. A holomorphic function $f: \mathcal{H} \mapsto \mathbb{C}$ which satisfies

$$
\forall\left(\begin{array}{ll}
a & b \\
c & d
\end{array}\right) \in \Gamma_{0}(q), \forall z \in \mathcal{H}, \quad f\left(\frac{a z+b}{c z+d}\right)=(c z+d)^{\kappa} f(z)
$$

and vanishes at the cusps of $\Gamma_{0}(q)$ is a holomorphic cusp form of level $q$, even weight $\kappa \geqslant 2$. We denote by $S_{\kappa}(q)$ this space of holomorphic cusp forms which is equipped with the Peterson inner product

$$
\left\langle f_{1}, f_{2}\right\rangle_{q}:=\int_{\Gamma_{0}(q) \backslash \mathcal{H}} y^{\kappa} f_{1}(z) \overline{f_{2}(z)} \frac{\mathrm{d} x \mathrm{~d} y}{y^{2}} .
$$


The Fourier expansion at the cusp $\infty$ of any such holomorphic cusp form $f$ is given by

$$
\forall z \in \mathcal{H}, \quad f(z)=\sum_{n \geqslant 1} \psi_{f}(n) n^{(\kappa-1) / 2} e(n z)
$$

where $e(z):=\exp (2 i \pi z)$ for any complex number $z$. The Hecke operators act on $S_{\kappa}(q)$ by

$$
T_{\ell}(f)(z):=\frac{1}{\sqrt{\ell}} \sum_{\substack{a d=\ell \\(a, q)=1}} \sum_{0 \leqslant b<d} f\left(\frac{a z+b}{d}\right)
$$

for any $z \in \mathcal{H}$. If $f$ is an eigenvector of $T_{\ell}$, we write $\lambda_{f}(\ell)$ the corresponding eigenvalue. We can prove that $T_{\ell}$ is hermitian if $\ell \geqslant 1$ is any integer coprime with $q$ and that

$$
T_{\ell_{1}} \circ T_{\ell_{2}}=\sum_{\substack{d \mid\left(\ell_{1}, \ell_{2}\right) \\(d, q)=1}} T_{\ell_{1} \ell_{2} / d^{2}}
$$

for any integers $\ell_{1}, \ell_{2} \geqslant 1$. By Atkin \& Lehner theory [1], we get a splitting of $S_{\kappa}(q)$ into $S_{\kappa}^{\mathrm{o}}(q) \oplus^{\perp\langle, \cdot\rangle_{q}} S_{\kappa}^{\mathrm{n}}(q)$ where

$$
\begin{aligned}
& S_{\kappa}^{\mathrm{o}}(q):=\operatorname{Vect}_{\mathbb{C}}\left\{f(q z), f \in S_{\kappa}(1)\right\} \cup S_{\kappa}(1), \\
& S_{\kappa}^{\mathrm{n}}(q):=\left(S_{\kappa}^{\mathrm{o}}(q)\right)^{\perp\langle\cdot, \cdot\rangle q}
\end{aligned}
$$

where "o" stands for "old" and "n" for "new". Note that $S_{\kappa}^{\mathrm{o}}(q)=\{0\}$ if $\kappa<12$ or $\kappa=14$. These two spaces are $T_{\ell^{-}}$invariant for any integer $\ell \geqslant 1$ coprime with $q$. A primitive cusp form $f \in S_{\kappa}^{\mathrm{n}}(q)$ is an eigenfunction of any operator $T_{\ell}$ for any integer $\ell \geqslant 1$ coprime with $q$ which is new and arithmetically normalised namely $\psi_{f}(1)=1$. Such an element $f$ is automatically an eigenfunction of the other Hecke operators and satisfies $\psi_{f}(\ell)=\lambda_{f}(\ell)$ for any integer $\ell \geqslant 1$. Moreover, if $p$ is a prime number, define $\alpha_{f}(p), \beta_{f}(p)$ as the complex roots of the quadratic equation

$$
X^{2}-\lambda_{f}(p) X+\varepsilon_{q}(p)=0
$$

where $\varepsilon_{q}$ denotes the trivial Dirichlet character of modulus $q$. Then it follows from the work of Eichler, Shimura, Igusa and Deligne that

$$
\left|\alpha_{f}(p)\right|,\left|\beta_{f}(p)\right| \leqslant 1
$$

for any prime number $p$ and so

$$
\forall \ell \geqslant 1, \quad\left|\lambda_{f}(\ell)\right| \leqslant \tau(\ell) .
$$

The set of primitive cusp forms is denoted by $H_{\kappa}^{*}(q)$. It is an orthogonal basis of $S_{\kappa}^{\mathrm{n}}(q)$. Let $f$ be a holomorphic cusp form with Hecke eigenvalues $\left(\lambda_{f}(\ell)\right)_{(\ell, q)=1}$. The composition property (2.1) entails that for any integer $\ell_{1} \geqslant 1$ and for any integer $\ell_{2} \geqslant 1$ coprime with $q$ the following multiplicative 
relations hold:

$$
\begin{aligned}
\psi_{f}\left(\ell_{1}\right) \lambda_{f}\left(\ell_{2}\right) & =\sum_{\substack{d \mid\left(\ell_{1}, \ell_{2}\right) \\
(d, q)=1}} \psi_{f}\left(\ell_{1} \ell_{2} / d^{2}\right) \\
\psi_{f}\left(\ell_{1} \ell_{2}\right) & =\sum_{\substack{d \mid\left(\ell_{1}, \ell_{2}\right) \\
(d, q)=1}} \mu(d) \psi_{f}\left(\ell_{1} / d\right) \lambda_{f}\left(\ell_{2} / d\right)
\end{aligned}
$$

and these relations hold for any integers $\ell_{1}, \ell_{2} \geqslant 1$ if $f$ is primitive. The adjointness relation is

$$
\lambda_{f}(\ell)=\overline{\lambda_{f}(\ell)}, \quad \psi_{f}(\ell)=\overline{\psi_{f}(\ell)}
$$

for any integer $\ell \geqslant 1$ coprime with $q$ and this remains true for any integer $\ell \geqslant 1$ if $f$ is primitive.

Trace formulas - We need two definitions. The harmonic weight associated to any $f$ in $S_{\kappa}(q)$ is defined by

$$
\omega_{q}(f):=\frac{\Gamma(\kappa-1)}{(4 \pi)^{\kappa-1}\langle f, f\rangle_{q}} .
$$

For any natural integer $m$ and $n$, the $\Delta_{q}$-symbol is given by

$$
\Delta_{q}(m, n):=\delta_{m, n}+2 \pi i^{\kappa} \sum_{\substack{c \geqslant 1 \\ q \mid c}} \frac{S(m, n ; c)}{c} J_{\kappa-1}\left(\frac{4 \pi \sqrt{m n}}{c}\right)
$$

where $S(m, n ; c)$ is a Kloosterman sum defined in appendix A.3 and $J_{\kappa-1}$ is a Bessel function of first kind defined in appendix A.2.The following proposition is Peterson's trace formula.

Proposition 2.1- If $H_{\kappa}(q)$ is any orthogonal basis of $S_{\kappa}(q)$ then

$$
\sum_{f \in H_{\kappa}(q)} \omega_{q}(f) \psi_{f}(m) \psi_{f}(n)=\Delta_{q}(m, n)
$$

for any integers $m$ and $n$.

H. Iwaniec, W. Luo \& P. Sarnak proved in [18] a useful variation of Peterson's trace formula which is an average over only primitive cusp forms. This is more convenient when there are some old forms which is the case for instance when the weight $\kappa$ is large. Let $\nu$ be the arithmetic function defined by

$$
\nu(n):=n \prod_{p \mid n}(1+1 / p)
$$

for any integer $n \geqslant 1$.

Proposition $2.2\left(\mathrm{H}\right.$. Iwaniec, W. Luo \& P. Sarnak (2001))- If $\left(n, q^{2}\right) \mid q$ and $q \nmid m$ then

$$
\sum_{f \in H_{\kappa}^{*}(q)} \omega_{q}(f) \lambda_{f}(m) \lambda_{f}(n)=\Delta_{q}(m, n)-\frac{1}{q \nu((n, q))} \sum_{\ell \mid q^{\infty}} \frac{1}{\ell} \Delta_{1}\left(m \ell^{2}, n\right) .
$$


Remark 2.3- The first term in (2.10) is exactly the term which appears in (2.9) whereas the second term in (2.10) will be usually very small as an old form comes from a form of level 1! Thus, everything works in practice as if there were no old forms in $S_{\kappa}(q)$.

2.1.2. Chebyshev polynomials and Hecke eigenvalues. Let $p \neq q$ a prime number and $f \in H_{\kappa}^{*}(q)$. The multiplicativity relation (2.4) leads to

$$
\sum_{r \geqslant 0} \lambda_{f}\left(p^{r}\right) t^{r}=\frac{1}{1-\lambda_{f}(p) t+t^{2}}
$$

It follows that

$$
\lambda_{f}\left(p^{r}\right)=X_{r}\left(\lambda_{f}(p)\right)
$$

where the polynomials $X_{r}$ are defined by their generating series

$$
\sum_{r \geqslant 0} X_{r}(x) t^{r}=\frac{1}{1-x t+t^{2}}
$$

They are also defined by

$$
X_{r}(2 \cos \theta)=\frac{\sin ((r+1) \theta)}{\sin (\theta)} .
$$

These polynomials are known as the Chebyshev polynomials of second kind. Each $X_{r}$ has degree $r$, is even if $r$ is even and odd otherwise. The family $\left\{X_{r}\right\}_{r \geqslant 0}$ is a basis for $\mathbb{Q}[X]$, orthonormal with respect to the inner product

$$
\langle P, Q\rangle_{\mathrm{ST}}:=\frac{1}{\pi} \int_{-2}^{2} P(x) Q(x) \sqrt{1-\frac{x^{2}}{4}} \mathrm{~d} x .
$$

In particular, for any integer $\varpi \geqslant 0$ we have

$$
X_{r}^{\varpi}=\sum_{j=0}^{r \varpi} x(\varpi, r, j) X_{j}
$$

with

$$
x(\varpi, r, j):=\left\langle X_{r}^{\varpi}, X_{j}\right\rangle_{\mathrm{ST}}=\frac{2}{\pi} \int_{0}^{\pi} \frac{\sin ^{\varpi}((r+1) \theta) \sin ((j+1) \theta)}{\sin ^{\varpi-1}(\theta)} \mathrm{d} \theta .
$$

The following relations are useful in this paper

$$
x(\varpi, r, j)= \begin{cases}1 & \text { if } j=0 \text { and } \varpi \text { is even, } \\ 0 & \text { if } j \text { is odd and } r \text { is even, } \\ 0 & \text { if } j=0, \varpi=1 \text { and } r \geqslant 1 .\end{cases}
$$

2.1.3. Overview of $L$-functions associated to primitive cusp forms. Let $f$ in $H_{\kappa}^{*}(q)$. We define

$$
L(f, s):=\sum_{n \geqslant 1} \frac{\lambda_{f}(n)}{n^{s}}=\prod_{p \in \mathcal{P}}\left(1-\frac{\alpha_{f}(p)}{p^{s}}\right)^{-1}\left(1-\frac{\beta_{f}(p)}{p^{s}}\right)^{-1}
$$

which is an absolutely convergent and non-vanishing Dirichlet series and Euler product on $\Re e s>1$ and also

$$
L_{\infty}(f, s):=\Gamma_{\mathbb{R}}(s+(\kappa-1) / 2) \Gamma_{\mathbb{R}}(s+(\kappa+1) / 2)
$$


where $\Gamma_{\mathbb{R}}(s):=\pi^{-s / 2} \Gamma(s / 2)$ as usual. The function

$$
\Lambda(f, s):=q^{s / 2} L_{\infty}(f, s) L(f, s)
$$

is a completed L-function in the sense that it satisfies the following nice analytic properties:

- the function $\Lambda(f, s)$ can be extended to an holomorphic function of order 1 on $\mathbb{C}$,

- the function $\Lambda(f, s)$ satisfies a functional equation of the shape

$$
\Lambda(f, s)=i^{\kappa} \varepsilon_{f}(q) \Lambda(f, 1-s)
$$

where

$$
\varepsilon_{f}(q)=-\sqrt{q} \lambda_{f}(q)= \pm 1 .
$$

2.1.4. Overview of symmetric power L-functions. Let $f$ in $H_{\kappa}^{*}(q)$. For any natural integer $r \geqslant 1$, the symmetric $r$-th power associated to $f$ is given by the following Euler product of degree $r+1$

$$
L\left(\operatorname{Sym}^{r} f, s\right):=\prod_{p \in \mathcal{P}} L_{p}\left(\operatorname{Sym}^{r} f, s\right)
$$

where

$$
L_{p}\left(\operatorname{Sym}^{r} f, s\right):=\prod_{i=0}^{r}\left(1-\frac{\alpha_{f}(p)^{i} \beta_{f}(p)^{r-i}}{p^{s}}\right)^{-1}
$$

for any prime number $p$. Let us remark that the local factors of this Euler product may be written as

$$
L_{p}\left(\operatorname{Sym}^{r} f, s\right)=\prod_{i=0}^{r}\left(1-\frac{\alpha_{f}(p)^{2 i-r}}{p^{s}}\right)^{-1}
$$

for any prime number $p \neq q$ and

$$
L_{q}\left(\operatorname{Sym}^{r} f, s\right)=1-\frac{\lambda_{f}(q)^{r}}{q^{s}}=1-\frac{\lambda_{f}\left(q^{r}\right)}{q^{s}}
$$

as $\alpha_{f}(p)+\beta_{f}(p)=\lambda_{f}(p)$ and $\alpha_{f}(p) \beta_{f}(p)=\varepsilon_{q}(p)$ for any prime number $p$ according to (2.2). On Res $>1$, this Euler product is absolutely convergent and non-vanishing. We also defines $[4,(3.16)$ and (3.17)] a local factor at $\infty$ which is given by a product of $r+1$ Gamma factors namely

$$
L_{\infty}\left(\operatorname{Sym}^{r} f, s\right):=\prod_{0 \leqslant a \leqslant(r-1) / 2} \Gamma_{\mathbb{R}}(s+(2 a+1)(\kappa-1) / 2) \Gamma_{\mathbb{R}}(s+1+(2 a+1)(\kappa-1) / 2)
$$

if $r$ is odd and

$$
L_{\infty}\left(\operatorname{Sym}^{r} f, s\right):=\Gamma_{\mathbb{R}}\left(s+\mu_{\kappa, r}\right) \prod_{1 \leqslant a \leqslant r / 2} \Gamma_{\mathbb{R}}(s+a(\kappa-1)) \Gamma_{\mathbb{R}}(s+1+a(\kappa-1))
$$

if $r$ is even where

$$
\mu_{\kappa, r}:= \begin{cases}1 & \text { if } r(\kappa-1) / 2 \text { is odd }, \\ 0 & \text { otherwise. }\end{cases}
$$

All the local data appearing in these local factors are encapsulated in the following completed $L$-function

$$
\Lambda\left(\operatorname{Sym}^{r} f, s\right):=\left(q^{r}\right)^{s / 2} L_{\infty}\left(\operatorname{Sym}^{r} f, s\right) L\left(\operatorname{Sym}^{r} f, s\right) .
$$


Here, $q^{r}$ is called the arithmetic conductor of $\Lambda\left(\operatorname{Sym}^{r} f, s\right)$ and somehow measures the size of this function. We will need some control on the analytic behaviour of this function. Unfortunately, such information is not currently known in all generality. Our main assumption is given in hypothesis Nice $(r, f)$ page 2 . Indeed, much more is expected to hold as it is discussed in details in [4] namely the following assumption is strongly believed to be true and lies in the spirit of Langlands program.

Hypothesis $\operatorname{Sym}^{r}(f)$ - There exists an automorphic cuspidal self-dual representation, denoted by $\operatorname{Sym}^{r} \pi_{f}=\otimes_{p \in \mathcal{P} \cup\{\infty\}}^{\prime} \operatorname{Sym}^{r} \pi_{f, p}$, of $G L_{r+1}\left(\mathbb{A}_{\mathbb{Q}}\right)$ whose local factors $L\left(\mathrm{Sym}^{r} \pi_{f, p}, s\right)$ agree with the local factors $L_{p}\left(\mathrm{Sym}^{r} f, s\right)$ for any $p$ in $\mathcal{P} \cup\{\infty\}$.

Note that the local factors and the arithmetic conductor in the definition of $\Lambda\left(\operatorname{Sym}^{r} f, s\right)$ and also the sign of its functional equation which all appear without any explanations so far come from the explicit computations which have been done via the local Langlands correspondence by J. Cogdell and P. Michel in [4]. Obviously, hypothesis $\operatorname{Nice}(r, f)$ is a weak consequence of hypothesis $\operatorname{Sym}^{r}(f)$. For instance, the cuspidality condition in hypothesis $\operatorname{Sym}^{r}(f)$ entails the fact that $\Lambda\left(\operatorname{Sym}^{r} f, s\right)$ is of order 1 which is crucial for us to state a suitable explicit formula. As we will not exploit the power of automorphic theory in this paper, hypothesis $\operatorname{Nice}(r, f)$ is enough for our purpose. In addition, it may happen that hypothesis Nice $(r, f)$ is known whereas hypothesis $\operatorname{Sym}^{r} f$ is not. Let us overview what has been done so far. For any $f$ in $H_{\kappa}^{*}(q)$, hypothesis $\operatorname{Sym}^{r} f$ is known for $r=1$ (E. Hecke), $r=2$ thanks to the work of S. Gelbart and H. Jacquet [8] and $r=3,4$ from the works of H. Kim and F. Shahidi [20-22].

2.2. Probabilistic background. The set $H_{\kappa}^{*}(q)$ can be seen as a probability space if

- the measurable sets are all its subsets,

- the harmonic probability measure is defined by

$$
\mu_{q}^{\mathrm{h}}(A):=\sum_{f \in A}^{\mathrm{h}} 1:=\sum_{f \in A} \omega_{q}(f)
$$

for any subset $A$ of $H_{\kappa}^{*}(q)$.

Indeed, there is a slight abuse here as we only know that

$$
\lim _{\substack{q \in \mathcal{P} \\ q \rightarrow+\infty}} \mu_{q}^{\mathrm{h}}\left(H_{\kappa}^{*}(q)\right)=1
$$

(see remark 3.12) which means that $\mu_{q}^{\mathrm{h}}$ is an "asymptotic" probability measure. If $X_{q}$ is a measurable complex-valued function on $H_{\kappa}^{*}(q)$ then it is very natural to compute its expectation defined by

$$
\mathbb{E}_{q}^{\mathrm{h}}\left(X_{q}\right):=\sum_{f \in H_{\kappa}^{*}(q)}^{\mathrm{h}} X_{q}(f)
$$

its variance defined by

$$
\mathbb{V}_{q}^{\mathrm{h}}\left(X_{q}\right):=\mathbb{E}_{q}^{\mathrm{h}}\left(\left(X_{q}-\mathbb{E}_{q}^{\mathrm{h}}\left(X_{q}\right)\right)^{2}\right)
$$


and its $m$-th moments given by

$$
\mathbb{M}_{q, m}^{\mathrm{h}}\left(X_{q}\right):=\mathbb{E}_{q}^{\mathrm{h}}\left(\left(X_{q}-\mathbb{E}_{q}^{\mathrm{h}}\left(X_{q}\right)\right)^{m}\right)
$$

for any integer $m \geqslant 1$. If $X:=\left(X_{q}\right)_{q \in \mathcal{P}}$ is a sequence of such measurable complex-valued functions then we may legitimely wonder if the associated complex sequences

$$
\left(\mathbb{E}_{q}^{\mathrm{h}}\left(X_{q}\right)\right)_{q \in \mathcal{P}}, \quad\left(\mathbb{V}_{q}^{\mathrm{h}}\left(X_{q}\right)\right)_{q \in \mathcal{P}}, \quad\left(\mathbb{M}_{q, m}^{\mathrm{h}}\left(X_{q}\right)\right)_{q \in \mathcal{P}}
$$

converge as $q$ goes to infinity among the primes. If yes, the following general notations will be used for their limits

$$
\mathbb{E}_{\infty}^{\mathrm{h}}(X), \quad \mathbb{V}_{\infty}^{\mathrm{h}}(X), \quad \mathbb{M}_{\infty, m}^{\mathrm{h}}(X)
$$

for any natural integer $m$. In addition, these potential limits are called asymptotic expectation, asymptotic variance and asymptotic $m$-th moments of $X$ for any natural integer $m \geqslant 1$.

For the end of this section, we assume that $r$ is odd. We may remark that the sign of the functional equations of any $L\left(\operatorname{Sym}^{r} f, s\right)$ when $q$ goes to infinity among the prime numbers and $f$ ranges over $H_{\kappa}^{*}(q)$ is not constant as it depends on $\varepsilon_{f}(q)$. Let

$$
H_{\kappa}^{\varepsilon}(q):=\left\{f \in H_{\kappa}^{*}(q), \varepsilon\left(\operatorname{Sym}^{r} f\right)=\varepsilon\right\}
$$

where $\varepsilon= \pm 1$. If $f \in H_{\kappa}^{+1}(q)$, then $\operatorname{Sym}^{r} f$ is said to be even whereas it is said to be odd if $f \in H_{\kappa}^{-1}(q)$. It is well-known that

$$
\lim _{\substack{q \in \mathcal{P} \\ q \rightarrow+\infty}} \mu_{q}^{\mathrm{h}}\left(\left\{f \in H_{k}^{*}(q): \varepsilon_{f}(q)=\varepsilon\right\}\right)=\frac{1}{2} .
$$

Since $\varepsilon\left(\operatorname{Sym}^{r} f\right)$ is $\varepsilon_{q}(f)$ up to a sign depending only on $\kappa$ and $r$ (by hypothesis Nice $(r, f))$, it follows that

$$
\lim _{\substack{q \in \mathcal{P} \\ q \rightarrow+\infty}} \mu_{q}^{\mathrm{h}}\left(H_{\kappa}^{\varepsilon}(q)\right)=\frac{1}{2} .
$$

For $X_{q}$ as previous, we can compute its signed expectation defined by

$$
\mathbb{E}_{q}^{\mathrm{h}, \varepsilon}\left(X_{q}\right):=2 \sum_{f \in H_{\kappa}^{\varepsilon}(q)}^{\mathrm{h}} X_{q}(f),
$$

its signed variance defined by

$$
\mathbb{V}_{q}^{\mathrm{h}, \varepsilon}\left(X_{q}\right):=\mathbb{E}_{q}^{\mathrm{h}, \varepsilon}\left(\left(X_{q}-\mathbb{E}_{q}^{\mathrm{h}, \varepsilon}\left(X_{q}\right)\right)^{2}\right)
$$

and its signed $m$-th moments given by

$$
\mathbb{M}_{q, m}^{\mathrm{h}, \varepsilon}\left(X_{q}\right):=\mathbb{E}_{q}^{\mathrm{h}, \varepsilon}\left(\left(X_{q}-\mathbb{E}_{q}^{\mathrm{h}, \varepsilon}\left(X_{q}\right)\right)^{m}\right)
$$

for any natural integer $m \geqslant 1$. In case of existence, we write $\mathbb{E}_{\infty}^{\mathrm{h}, \varepsilon}(X), \mathbb{V}_{\infty}^{\mathrm{h}, \varepsilon}(X)$ and $\mathbb{M}_{\infty, m}^{\mathrm{h}, \varepsilon}(X)$ for the limits which are called signed asymptotic expectation, 
signed asymptotic variance and signed asymptotic moments. The signed expectation and the expectation are linked through the formula

$$
\begin{aligned}
\mathbb{E}_{q}^{\mathrm{h}, \varepsilon}\left(X_{q}\right) & =2 \sum_{f \in H_{\kappa}^{*}(q)}^{\mathrm{h}} \frac{1+\varepsilon \times \varepsilon\left(\mathrm{Sym}^{r} f\right)}{2} X_{q}(f) \\
& =\mathbb{E}_{q}^{\mathrm{h}}\left(X_{q}\right)-\varepsilon \times \varepsilon(\kappa, r) \sqrt{q} \sum_{f \in H_{\kappa}^{*}(q)}^{\mathrm{h}} \lambda_{f}(q) X_{q}(f) .
\end{aligned}
$$

\section{MAin teChNicAl ingREDiEntS OF THIS WORK}

3.1. Large sieve inequalities for Kloosterman sums. One of the main ingredients in this work is some large sieve inequalities for Kloosterman sums which have been established by J.-M. Deshouillers \& H. Iwaniec in [5] and then refined by V. Blomer, G. Harcos \& P. Michel in [2]. The proof of these large sieve inequalities relies on the spectral theory of automorphic forms on $G L_{2}\left(\mathbb{A}_{\mathbb{Q}}\right)$. In particular, the authors have to understand the size of the Fourier coefficients of these automorphic cusp forms. We have already seen that the size of the Fourier coefficients of holomorphic cusp forms is well understood (2.3) but we only have partial results on the size of the Fourier coefficients of Maass cusp forms which do not come from holomorphic forms. We introduce the following hypothesis which measures the approximation towards the Ramanujan-Peterson-Selberg conjecture.

Hypothesis $\mathrm{H}_{2}(\theta)-$ If $\pi:=\otimes_{p \in \mathcal{P} \cup\{\infty\}}^{\prime} \pi_{p}$ is any automorphic cuspidal form on $G L_{2}\left(\mathbb{A}_{\mathbb{Q}}\right)$ with local Hecke parameters $\alpha_{\pi}^{(1)}(p), \alpha_{\pi}^{(2)}(p)$ at any prime number $p$ and $\mu_{\pi}^{(1)}(\infty), \mu_{\pi}^{(2)}(\infty)$ at infinity then

$$
\forall j \in\{1,2\}, \quad\left|\alpha_{\pi}^{(j)}(p)\right| \leqslant p^{\theta}
$$

for any prime number $p$ for which $\pi_{p}$ is unramified and

$$
\forall j \in\{1,2\}, \quad\left|\Re e\left(\mu_{\pi}^{(j)}(\infty)\right)\right| \leqslant \theta
$$

provided $\pi_{\infty}$ is unramified.

Definition 3.1- We say that $\theta$ is admissible if $\mathrm{H}_{2}(\theta)$ is satisfied.

Remark 3.2- The smallest admissible value of $\theta$ is currently $\theta_{0}=\frac{7}{64}$ thanks to the works of H. Kim, F. Shahidi and P. Sarnak [20,21]. The RamanujanPeterson-Selberg conjecture asserts that 0 is admissible.

Definition 3.3- Let $T: \mathbb{R}^{3} \rightarrow \mathbb{R}^{+}$and $(M, N, C) \in(\mathbb{R} \backslash\{0\})^{3}$, we say that a smooth function $h: \mathbb{R}^{3} \rightarrow \mathbb{R}^{3}$ satisfies the property $\mathrm{P}(T ; M, N, C)$ if there exists a real number $K>0$ such that

$$
\begin{gathered}
\forall(i, j, k) \in \mathbb{N}^{3}, \forall\left(x_{1}, x_{2}, x_{3}\right) \in\left[\frac{M}{2}, 2 M\right] \times\left[\frac{N}{2}, 2 N\right] \times\left[\frac{C}{2}, 2 C\right] \\
x_{1}^{i} x_{2}^{j} x_{3}^{k} \frac{\partial^{i+j+k} h}{\partial x_{1}^{i} \partial x_{2}^{j} \partial x_{3}^{k}}\left(x_{1}, x_{2}, x_{3}\right) \leqslant K T(M, N, C)\left(1+\frac{\sqrt{M N}}{C}\right)^{i+j+k} .
\end{gathered}
$$


With this definition in mind, we are able to write the following proposition which is special case of a large sieve inequality adapted from the one of Deshouillers \& Iwaniec [5, Theorem 9] by Blomer, Harcos \& Michel [2, Theorem 4].

Proposition 3.4- Let $q$ be some positive integer. Let $M, N, C \geqslant 1$ and $g$ be a smooth function satisfying property $\mathrm{P}(1 ; M, N, C)$. Consider two sequences

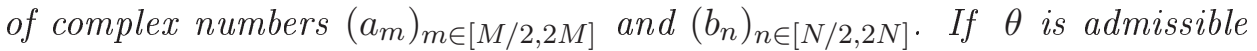
and $M N \ll C^{2}$ then

$$
\begin{aligned}
\sum_{\substack{c \geqslant 1 \\
q \mid c}} \sum_{m \geqslant 1} \sum_{n \geqslant 1} a_{m} b_{n} \frac{S(m, \pm n ; c)}{c} g(m, n ; c) \\
\quad \ll_{\varepsilon}(q M N C)^{\varepsilon}\left(\frac{C^{2}}{M N}\right)^{\theta}\left(1+\frac{M}{q}\right)^{1 / 2}\left(1+\frac{N}{q}\right)^{1 / 2}\|a\|_{2}\|b\|_{2}
\end{aligned}
$$

for any $\varepsilon>0$.

We shall use a test function. For any $\nu>0$ let us define $\mathcal{S}_{\nu}(\mathbb{R})$ as the space of even Schwartz function $\Phi$ whose Fourier transform

$$
\widehat{\Phi}(\xi):=\mathcal{F}[x \mapsto \Phi(x)](\xi):=\int_{\mathbb{R}} \Phi(x) e(-x \xi) \mathrm{d} x
$$

is compactly supported in $[-\nu,+\nu]$. Thanks to the Fourier inversion formula:

$$
\Phi(x)=\int_{\mathbb{R}} \widehat{\Phi}(\xi) e(x \xi) \mathrm{d} x=\mathcal{F}[\xi \mapsto \widehat{\Phi}(\xi)](-x),
$$

such a function $\Phi$ can be extended to an entire even function which satisfies

$$
\forall s \in \mathbb{C}, \quad \Phi(s) \ll_{n} \frac{\exp (\nu|\Im m s|)}{(1+|s|)^{n}}
$$

for any integer $n \geqslant 0$. The version of the large sieve inequality we shall use several times in this paper is then the following.

Corollary 3.5-Let $q$ be some prime number, $k_{1}, k_{2}>0$ be some integers, $\alpha_{1}, \alpha_{2}, \nu$ be some positive real numbers and $\Phi \in \mathcal{S}_{\nu}(\mathbb{R})$. Let $h$ be some smooth function satisfying property $\mathrm{P}(T ; M, N, C)$ for any $1 \leqslant M \leqslant q^{k_{1} \alpha_{1} \nu}$, $1 \leqslant N \leqslant q^{k_{2} \alpha_{2} \nu}$ and $C \geqslant q$. Let $\left(a_{p}\right)_{\substack{p \in \mathcal{P} \\ p \leqslant q^{\alpha_{1} \nu}}}$ and $\left(b_{p}\right)_{\substack{p \in \mathcal{P} \\ p \leqslant q^{\alpha_{2} \nu}}}$ be some complex numbers sequences. If $\theta$ is admissible and $\nu \leqslant 2 /\left(k_{1} \alpha_{1}+k_{2} \alpha_{2}\right)$ then

$$
\begin{aligned}
& \sum_{\substack{c \geqslant 1 \\
q \mid c}} \sum_{\substack{p_{1} \in \mathcal{P} \\
p_{1} \nmid q}} \sum_{\substack{p_{2} \in \mathcal{P} \\
p_{2} \nmid q}} a_{p_{1}} b_{p_{2}} \frac{S\left(p_{1}^{k_{1}}, p_{2}^{k_{2}} ; c\right)}{c} h\left(p_{1}^{k_{1}}, p_{2}^{k_{2}} ; c\right) \widehat{\Phi}\left(\frac{\log p_{1}}{\log \left(q^{\alpha_{1}}\right)}\right) \widehat{\Phi}\left(\frac{\log p_{2}}{\log \left(q^{\alpha_{2}}\right)}\right)
\end{aligned}
$$

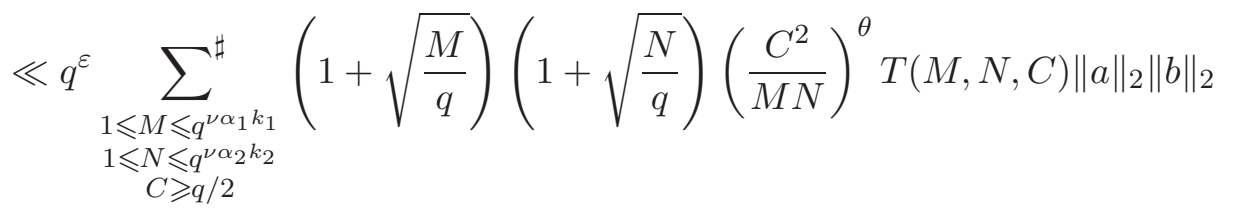

where $\sharp$ indicates that the sum is on powers of $\sqrt{2}$. The constant implied by the symbol « depends at most on $\varepsilon, k_{1}, k_{2}, \alpha_{1}, \alpha_{2}$ and $\nu$. 
Proof. Define $\left(\widehat{a}_{m}\right)_{m \in \mathbb{N}},\left(\widehat{b}_{n}\right)_{n \in \mathbb{N}}$ and $g(m, n ; c)$ by

$$
\begin{aligned}
\widehat{a}_{m} & :=a_{m^{1 / k_{1}}} 1_{\mathcal{P}^{k_{1}}}(m) 1_{\left[1, q^{\left.\nu \alpha_{1} k_{1}\right]}\right.}(m) \\
\widehat{b}_{n} & :=b_{n^{1 / k_{1}}} 1_{\mathcal{P}^{k_{1}}}(n) 1_{\left[1, q^{\left.\nu \alpha_{1} k_{1}\right]}\right.}(n) \\
g(m, n ; c) & :=h(m, n, c) \widehat{\Phi}\left(\frac{\log m}{\log \left(q^{\alpha_{1} k_{1}}\right)}\right) \widehat{\Phi}\left(\frac{\log n}{\log \left(q^{\alpha_{2} k_{2}}\right)}\right) .
\end{aligned}
$$

Using a smooth partition of unity, as detailed in $\S$ A.1, we need to evaluate

$$
\sum_{\substack{1 \leqslant M \leqslant q^{\nu \alpha_{1} k_{1}} \\ 1 \leqslant N \leqslant q^{\nu \alpha_{2} k_{2}} \\ C \geqslant q / 2}}^{\sharp} T(M, N, C) \sum_{\substack{c \geqslant 1 \\ q \mid c}} \sum_{m \geqslant 1} \sum_{n \geqslant 1} \widehat{a}_{m} \widehat{b}_{n} \frac{S(m, n ; c)}{c} \frac{g_{M, N, C}(m, n ; c)}{T(M, N, C)} .
$$

Since $\nu \leqslant 2 /\left(\alpha_{1} k_{1}+\alpha_{2} k_{2}\right)$, the first summation is restricted to $M N \ll C^{2}$ hence, using proposition 3.4 , the quantity in (3.8) is

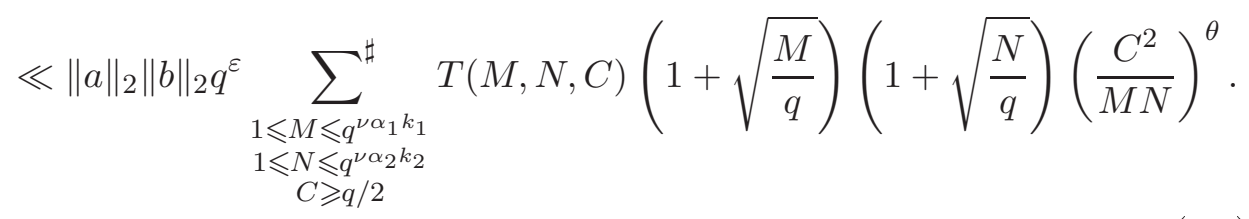

3.2. Riemann's explicit formula for symmetric power $L$-functions. In this section, we give an analog of Riemann-von Mangoldt's explicit formula for symmetric power $L$-functions. Before that, let us recall some preliminary facts on zeros of symmetric power $L$-functions which can be found in section 5.3 of [17]. Let $r \geqslant 1$ and $f \in H_{\kappa}^{*}(q)$ for which hypothesis Nice $(r, f)$ holds. All the zeros of $\Lambda\left(\operatorname{Sym}^{r} f, s\right)$ are in the critical strip $\{s \in \mathbb{C}: 0<\Re e s<1\}$. The multiset of the zeros of $\Lambda\left(\operatorname{Sym}^{r} f, s\right)$ counted with multiplicities is given by

where

$$
\left\{\rho_{f, r}^{(j)}=\beta_{f, r}^{(j)}+i \gamma_{f, r}^{(j)}: j \in \mathcal{E}(f, r)\right\}
$$

and

$$
\mathcal{E}(f, r):= \begin{cases}\mathbb{Z} & \text { if } \operatorname{Sym}^{r} f \text { is odd } \\ \mathbb{Z} \backslash\{0\} & \text { if } \operatorname{Sym}^{r} f \text { is even }\end{cases}
$$

$$
\begin{aligned}
& \beta_{f, r}^{(j)}=\Re e \rho_{f, r}^{(j)}, \\
& \gamma_{f, r}^{(j)}=\Im m \rho_{f, r}^{(j)}
\end{aligned}
$$

for any $j \in \mathcal{E}(f, r)$. We enumerate the zeros such that

(1) the sequence $j \mapsto \gamma_{f, r}^{(j)}$ is increasing

(2) we have $j \geqslant 0$ if and only if $\gamma_{f, r}^{(j)} \geqslant 0$

(3) we have $\rho_{f, r}^{(-j)}=1-\rho_{f, r}^{(j)}$.

Note that if $\rho_{f, r}^{(j)}$ is a zero of $\Lambda\left(\operatorname{Sym}^{r} f, s\right)$ then $\overline{\rho_{f, r}^{(j)}}, 1-\rho_{f, r}^{(j)}$ and $1-\overline{\rho_{f, r}^{(j)}}$ are also some zeros of $\Lambda\left(\operatorname{Sym}^{r} f, s\right)$. In addition, remember that if $\operatorname{Sym}^{r} f$ is odd then the functional equation of $L\left(\operatorname{Sym}^{r} f, s\right)$ evaluated at the critical point 
$s=1 / 2$ provides a trivial zero denoted by $\rho_{f, r}^{(0)}$. It can be shown $[17$, Theorem 5.8] that the number of zeros $\Lambda\left(\operatorname{Sym}^{r} f, s\right)$ satisfying $\left|\gamma_{f, r}^{(j)}\right| \leqslant T$ is

$$
\frac{T}{\pi} \log \left(\frac{q^{r} T^{r+1}}{(2 \pi e)^{r+1}}\right)+O(\log (q T))
$$

as $T \geqslant 1$ goes to infinity. We state now the Generalised Riemann Hypothesis which is the main conjecture about the horizontal distribution of the zeros of $\Lambda\left(\operatorname{Sym}^{r} f, s\right)$ in the critical strip.

Hypothesis $\operatorname{GRH}(r)-$ For any prime number $q$ and any $f$ in $H_{\kappa}^{*}(q)$, all the zeros of $\Lambda\left(\operatorname{Sym}^{r} f, s\right)$ lie on the critical line $\{s \in \mathbb{C}: \Re e s=1 / 2\}$ namely $\beta_{r, f}^{(j)}=1 / 2$ for any $j \in \mathcal{E}(f, r)$.

Remark 3.6- We do not use this hypothesis in our proofs.

Under hypothesis $\operatorname{GRH}(r)$, it can be shown that the number of zeros of the function $\Lambda\left(\operatorname{Sym}^{r} f, s\right)$ satisfying $\left|\gamma_{f, r}^{(j)}\right| \leqslant 1$ is given by

$$
\frac{1}{\pi} \log \left(q^{r}\right)(1+o(1))
$$

as $q$ goes to infinity. Thus, the spacing between two consecutive zeros with imaginary part in $[0,1]$ is roughly of size

$$
\frac{2 \pi}{\log \left(q^{r}\right)}
$$

We aim at studying the local distribution of the zeros of $\Lambda\left(\operatorname{Sym}^{r} f, s\right)$ in a neighborhood of the real axis of size $1 / \log q^{r}$ since in such a neighborhood, we expect to catch only few zeros (but without being able to say that we catch only one ${ }^{2}$ ). Hence, we normalise the zeros by defining

$$
\widehat{\rho}_{f, r}^{(j)}:=\frac{\log \left(q^{r}\right)}{2 i \pi}\left(\beta_{f, r}^{(j)}-\frac{1}{2}+i \gamma_{f, r}^{(j)}\right) .
$$

Note that

$$
\widehat{\rho}_{f, r}^{(-j)}=-\widehat{\rho}_{f, r}^{(j)} .
$$

Definition 3.7- Let $f \in H_{\kappa}^{*}(q)$ for which hypothesis $\operatorname{Nice}(r, f)$ holds and let $\Phi \in \mathcal{S}_{\nu}(\mathbb{R})$. The one-level density (relatively to $\Phi$ ) of $\mathrm{Sym}^{r} f$ is

$$
D_{1, q}[\Phi ; r](f):=\sum_{j \in \mathcal{E}(f, r)} \Phi\left(\widehat{\rho}_{f, r}^{(j)}\right) .
$$

To study $D_{1, q}[\Phi ; r](f)$ for any $\Phi \in \mathcal{S}_{\nu}(\mathbb{R})$, we transform this sum over zeros into a sum over primes in the next proposition. In other words, we establish an explicit formula for symmetric power $L$-functions. Since the proof is classical, we refer to $[18, \S 4]$ or $[9, \S 2.2]$ which present a method that has just to be adapted to our setting.

\footnotetext{
${ }^{2}$ We refer to Miller [25] and Omar [27] for works related to the "first" zero.
} 
Proposition 3.8- Let $r \geqslant 1$ and $f \in H_{\kappa}^{*}(q)$ for which hypothesis $\operatorname{Nice}(r, f)$ holds and let $\Phi \in \mathcal{S}_{\nu}(\mathbb{R})$. We have

$D_{1, q}[\Phi ; r](f)=E[\Phi ; r]+P_{q}^{1}[\Phi ; r](f)+\sum_{m=0}^{r-1}(-1)^{m} P_{q}^{2}[\Phi ; r, m](f)+O\left(\frac{1}{\log \left(q^{r}\right)}\right)$

where

$$
\begin{aligned}
E[\Phi ; r] & :=\widehat{\Phi}(0)+\frac{(-1)^{r+1}}{2} \Phi(0), \\
P_{q}^{1}[\Phi ; r](f) & :=-\frac{2}{\log \left(q^{r}\right)} \sum_{\substack{p \in \mathcal{P} \\
p \nmid q}} \lambda_{f}\left(p^{r}\right) \frac{\log p}{\sqrt{p}} \widehat{\Phi}\left(\frac{\log p}{\log \left(q^{r}\right)}\right), \\
P_{q}^{2}[\Phi ; r, m](f) & :=-\frac{2}{\log \left(q^{r}\right)} \sum_{\substack{p \in \mathcal{P} \\
p \nmid q}} \lambda_{f}\left(p^{2(r-m)}\right) \frac{\log p}{p} \widehat{\Phi}\left(\frac{2 \log p}{\log \left(q^{r}\right)}\right)
\end{aligned}
$$

for any integer $m \in\{0, \ldots, r-1\}$.

3.3. Contribution of the old forms. In this short section, we prove the following useful lemmas.

Lemma 3.9- Let $p_{1}$ and $p_{2} \neq q$ be some prime numbers and $a_{1}, a_{2}$, a be some nonnegative integers. Then

$$
\sum_{\ell \mid q^{\infty}} \frac{\Delta_{1}\left(\ell^{2} p_{1}^{a_{1}}, p_{2}^{a_{2}} q^{a}\right)}{\ell} \ll \frac{1}{q^{a / 2}}
$$

the implied constant depending only on $a_{1}$ and $a_{2}$.

Proof. Using proposition 2.1 and the fact that $H_{\kappa}(1)=H_{\kappa}^{*}(1)$, we write

$$
\begin{aligned}
\Delta_{1}\left(\ell^{2} p_{1}^{a_{1}}, p_{2}^{a_{2}} q^{a}\right) & =\sum_{f \in H_{\kappa}^{*}(1)}^{\mathrm{h}} \lambda_{f}\left(\ell^{2} p_{1}^{a_{1}}\right) \lambda_{f}\left(p_{2}^{a_{2}} q^{a}\right) \\
& \ll \sum_{f \in H_{\kappa}^{*}(1)}^{\mathrm{h}}\left|\lambda_{f}\left(\ell^{2} p_{1}^{a_{1}}\right)\right| \cdot\left|\lambda_{f}\left(p_{2}^{a_{2}}\right)\right| \cdot\left|\lambda_{f}\left(q^{a}\right)\right| .
\end{aligned}
$$

By Deligne's bound (2.3) we have

$$
\left|\lambda_{f}\left(\ell^{2} p_{1}^{a_{1}}\right)\right| \cdot\left|\lambda_{f}\left(p_{2}^{a_{2}}\right)\right| \leqslant \tau\left(\ell^{2} p_{1}^{a_{1}}\right) \tau\left(p_{2}^{a_{2}}\right) \leqslant\left(a_{1}+1\right)\left(a_{2}+2\right) \tau\left(\ell^{2}\right) .
$$

By the multiplicativity relation (2.4) and the value of the sign of the functional equation (2.15), we have

$$
\left|\lambda_{f}\left(q^{a}\right)\right| \ll \frac{1}{q^{a / 2}} .
$$

We obtain the result by reporting (3.16) and (3.15) in (3.14) and by using (2.16) and

$$
\sum_{\ell \mid q^{\infty}} \frac{\tau\left(\ell^{2}\right)}{\ell}=\frac{1+1 / q}{(1-1 / q)^{2}} \ll 1 .
$$


Lemma 3.10- Let $m, n \geqslant 1$ be some coprime integers. Then,

$$
\Delta_{q}(m, n)-\delta(m, n) \ll \begin{cases}\frac{(m n)^{1 / 4}}{q} \log \left(\frac{m n}{q^{2}}\right) & \text { if } m n>q^{2} \\ \frac{(m n)^{(\kappa-1) / 2}}{q^{\kappa-1 / 2}} \leqslant \frac{(m n)^{1 / 4}}{q} & \text { if } m n \leqslant q^{2} .\end{cases}
$$

Proof. This is a direct consequence of the Weil-Estermann bound (A.6) and lemma A.1.

Corollary 3.11- For any prime number q, we have

$$
\sqrt{q} \sum_{f \in H_{\kappa}^{*}(q)}^{\mathrm{h}} \lambda_{f}(q) \ll \frac{1}{q^{\delta_{\kappa}}}
$$

where

$$
\delta_{\kappa}:= \begin{cases}\frac{\kappa-1}{2} & \text { if } \kappa \leqslant 10 \text { or } \kappa=14 \\ \frac{5}{2} & \text { otherwise. }\end{cases}
$$

Proof of corollary 3.11. Let $\mathcal{K}=\{\kappa \in 2 \mathbb{N}: 2 \leqslant \kappa \leqslant 14, \kappa \neq 12\}$. By proposition 2.2 , we have

$$
\sum_{f \in H_{\kappa}^{*}(q)}^{\mathrm{h}} \lambda_{f}(q)=\Delta_{q}(1, q)-\frac{\delta(\kappa \notin \mathcal{K})}{q \nu(q)} \sum_{\ell \mid q^{\infty}} \frac{\Delta_{1}\left(\ell^{2}, q\right)}{\ell} .
$$

The term $\delta(\kappa \notin \mathcal{K})$ comes from proposition 2.1 with the fact that there is no cusp forms of weight $\kappa \in \mathcal{K}$ and level 1 . Lemma 3.10 gives

$$
\Delta_{q}(1, q) \ll \frac{1}{q^{\kappa / 2}}
$$

and lemma 3.9 gives

$$
\sum_{\ell \mid q^{\infty}} \frac{\Delta_{1}\left(\ell^{2}, q\right)}{\ell} \ll \frac{1}{\sqrt{q}} .
$$

Since $\nu(q)>q$, the result follows from reporting (3.18) and (3.19) in (3.17).

Remark 3.12- In a very similar fashion, one can prove that

$$
\mu_{q}^{\mathrm{h}}\left(H_{\kappa}^{*}(q)\right)=\mathbb{E}_{q}^{\mathrm{h}}(1)=1+O\left(\frac{1}{q^{\gamma_{\kappa}}}\right) .
$$

where

$$
\gamma_{\kappa}:= \begin{cases}\kappa-\frac{1}{2} & \text { if } \kappa \leqslant 10 \text { or } \kappa=14 \\ 1 & \text { otherwise. }\end{cases}
$$

Corollary 3.11, (3.20) and (2.18) imply

$$
\mathbb{E}_{q}^{\mathrm{h}, \varepsilon}(1)=1+O\left(\frac{1}{q^{\beta_{\kappa}}}\right)
$$

where

$$
\beta_{\kappa}:= \begin{cases}\frac{\kappa-1}{2} & \text { if } \kappa \leqslant 10 \text { or } \kappa=14 \\ 1 & \text { otherwise. }\end{cases}
$$

A direct consequence of lemma 3.9 is the following one. 
Lemma 3.13- Let $\alpha_{1}, \alpha_{2}, \beta_{1}, \beta_{2}, \gamma_{1}, \gamma_{2}, w$ be some nonnegative real numbers. Let $\Phi_{1}$ and $\Phi_{2}$ be in $\mathcal{S}_{\nu}(\mathbb{R})$. Then,

$$
\begin{aligned}
& \sum_{\substack{p_{1} \in \mathcal{P} \\
p_{1} \nmid q}} \sum_{p_{2} \in \mathcal{P}} \frac{\log p_{1} \nmid q}{p_{1}^{\alpha_{1}}} \frac{\log p_{2}}{p_{2}^{\alpha_{2}}} \widehat{\Phi_{1}}\left(\frac{\log p_{1}}{\log \left(q^{\beta_{1}}\right)}\right) \widehat{\Phi_{2}}\left(\frac{\log p_{2}}{\log \left(q^{\beta_{2}}\right)}\right) \sum_{\ell \mid q^{\infty}} \frac{\Delta_{1}\left(\ell^{2} p_{1}^{\gamma_{1}}, p_{2}^{\gamma_{2}} q^{w}\right)}{\ell} \\
& \ll q^{\delta \nu-w / 2+\varepsilon}
\end{aligned}
$$

with $\delta$ given in table 1.

\section{LinEAR STATISTICS FOR LOW-LYING ZEROS}

4.1. Density results for families of $L$-functions. We briefly recall some well-known features that can be found in [18]. Let $\mathcal{F}$ be a family of $L$ functions indexed by the arithmetic conductor namely

$$
\mathcal{F}=\bigcup_{Q \geqslant 1} \mathcal{F}(Q)
$$

where the arithmetic conductor of any $L$-function in $\mathcal{F}(Q)$ is of order $Q$ in the logarithmic scale. It is expected that there is a symmetry group $G(\mathcal{F})$ of matrices of large rank endowed with a probability measure which can be associated to $\mathcal{F}$ such that the low-lying zeros of the $L$-functions in $\mathcal{F}$ namely the non-trivial zeros of height less than $1 / \log Q$ are distributed like the eigenvalues of the matrices in $G(\mathcal{F})$. In other words, there should exist a symmetry group $G(\mathcal{F})$ such that for any $\nu>0$ and any $\Phi \in \mathcal{S}_{\nu}(\mathbb{R})$,

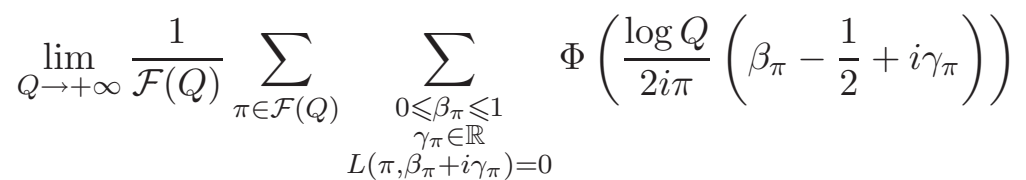

$$
\begin{aligned}
& =\int_{\mathbb{R}} \Phi(x) W_{1}(G(\mathcal{F}))(x) \mathrm{d} x
\end{aligned}
$$

where $W_{1}(G(\mathcal{F}))$ is the one-level density of the eigenvalues of $G(\mathcal{F})$. In this case, $\mathcal{F}$ is said to be of symmetry type $G(\mathcal{F})$ and we said that we proved a density result for $\mathcal{F}$. For instance, the following densities are determined in [19]:

$$
\begin{aligned}
W_{1}(S O(\text { even }))(x) & =1+\frac{\sin (2 \pi x)}{2 \pi x}, \\
W_{1}(O)(x) & =1+\frac{1}{2} \delta_{0}(x), \\
W_{1}(S O(\text { odd }))(x) & =1-\frac{\sin (2 \pi x)}{2 \pi x}+\delta_{0}(x), \\
W_{1}(S p)(x) & =1-\frac{\sin (2 \pi x)}{2 \pi x}
\end{aligned}
$$

where $\delta_{0}$ is the Dirac distribution at 0 . According to Plancherel's formula,

$$
\int_{\mathbb{R}} \Phi(x) W_{1}(G(\mathcal{F}))(x) \mathrm{d} x=\int_{\mathbb{R}} \widehat{\Phi}(x) \widehat{W}_{1}(G(\mathcal{F}))(x) \mathrm{d} x
$$


and we can check that

$$
\begin{aligned}
\widehat{W}_{1}(S O(\text { even }))(x) & =\delta_{0}(x)+\frac{1}{2} \eta(x), \\
\widehat{W}_{1}(O)(x) & =\delta_{0}(x)+\frac{1}{2} \\
\widehat{W}_{1}(S O(\text { odd }))(x) & =\delta_{0}(x)-\frac{1}{2} \eta(x)+1, \\
\widehat{W}_{1}(S p)(x) & =\delta_{0}(x)-\frac{1}{2} \eta(x)
\end{aligned}
$$

where

$$
\eta(x):= \begin{cases}1 & \text { if }|x|<1 \\ \frac{1}{2} & \text { if } x= \pm 1 \\ 0 & \text { otherwise }\end{cases}
$$

As a consequence, if we can only prove a density result for $\nu \leqslant 1$, the three orthogonal densities are indistinguishable although they are distinguishable from $S p$. Thus, the challenge is to pass the natural barrier $\nu=1$.

4.2. Asymptotic expectation of the one-level density. The aim of this part is to prove a density result for the family

$$
\mathcal{F}_{r}:=\bigcup_{q \in \mathcal{P}}\left\{L\left(\operatorname{Sym}^{r} f, s\right), f \in H_{\kappa}^{*}(q)\right\}
$$

for any $r \geqslant 1$ which consists in proving the existence and computing the asymptotic expectation $\mathbb{E}_{\infty}^{\mathrm{h}}\left(D_{1}[\Phi ; r]\right)$ of $D_{1}[\Phi ; r]:=\left(D_{1, q}[\Phi ; r]\right)_{q \in \mathcal{P}}$ for any $r \geqslant 1$ and for $\Phi$ in $\mathcal{S}_{\nu}(\mathbb{R})$ with $\nu>0$ as large as possible in order to be able to distinguish between the three orthogonal densities if $r$ is small enough. Recall that $E[\Phi ; r]$ has been defined in proposition 3.8.

Theorem 4.1- Let $r \geqslant 1$ and $\Phi \in \mathcal{S}_{\nu}(\mathbb{R})$. We assume that hypothesis Nice $(r, f)$ holds for any prime number $q$ and any $f \in H_{\kappa}^{*}(q)$ and also that $\theta$ is admissible. Let

$$
\nu_{1, \max }(r, \kappa, \theta):=\left(1-\frac{1}{2(\kappa-2 \theta)}\right) \frac{2}{r^{2}} .
$$

If $\nu<\nu_{1, \max }(r, \kappa, \theta)$ then

$$
\mathbb{E}_{\infty}^{\mathrm{h}}\left(D_{1}[\Phi ; r]\right)=E[\Phi ; r] .
$$

Remark 4.2- We remark that

$$
\begin{aligned}
& \nu_{1, \text { max }}\left(r, \kappa, \theta_{0}\right)=\left(1-\frac{16}{32 \kappa-7}\right) \frac{2}{r^{2}} \geqslant \frac{82}{57 r^{2}}, \\
& \nu_{1, \max }(r, \kappa, 0)=\left(1-\frac{1}{2 \kappa}\right) \frac{2}{r^{2}} \quad \geqslant \frac{3}{2 r^{2}}
\end{aligned}
$$

and thus $\nu_{1, \max }\left(1, \kappa, \theta_{0}\right)>1$ whereas $\nu_{1, \max }\left(r, \kappa, \theta_{0}\right) \leqslant 1$ for any $r \geqslant 2$.

Remark 4.3- Note that

$$
E[\Phi ; r]=\int_{\mathbb{R}} \widehat{\Phi}(x)\left(\delta_{0}(x)+\frac{(-1)^{r+1}}{2}\right) \mathrm{d} x .
$$


Thus, this theorem reveals that the symmetry type of $\mathcal{F}_{r}$ is

$$
G\left(\mathcal{F}_{r}\right)= \begin{cases}S p & \text { if } r \text { is even, } \\ O & \text { if } r=1, \\ S O(\text { even }) \text { or } O \text { or } S O(\text { odd }) & \text { if } r \geqslant 3 \text { is odd }\end{cases}
$$

Some additional comments are given in remark 4 page 5 .

Proof of theorem 4.1. The proof is detailed and will be a model for the next density results. According to proposition 3.8 and (3.20), we have

$$
\begin{aligned}
& \mathbb{E}_{q}^{\mathrm{h}}\left(D_{1, q}[\Phi ; r]\right)=E[\Phi ; r]+\mathbb{E}_{q}^{\mathrm{h}}\left(P_{q}^{1}[\Phi ; r]\right) \\
& +\sum_{m=0}^{r-1}(-1)^{m} \mathbb{E}_{q}^{\mathrm{h}}\left(P_{q}^{2}[\Phi ; r, m]\right)+O\left(\frac{1}{\log \left(q^{r}\right)}\right) .
\end{aligned}
$$

The first term in (4.3) is the main term given in the theorem. We now estimate the second term of (4.3) via the trace formula given in proposition 2.2 .

$$
\mathbb{E}_{q}^{\mathrm{h}}\left(P_{q}^{1}[\Phi ; r]\right)=\mathbb{P}_{q, \text { new }}^{1}[\Phi ; r]+\mathbb{P}_{q, \text { old }}^{1}[\Phi ; r]
$$

where

$$
\begin{aligned}
\mathbb{P}_{q, \text { new }}^{1}[\Phi ; r] & =-\frac{2}{\log \left(q^{r}\right)} \sum_{\substack{p \in \mathcal{P} \\
p \nmid q}} \Delta_{q}\left(p^{r}, 1\right) \frac{\log p}{\sqrt{p}} \widehat{\Phi}\left(\frac{\log p}{\log \left(q^{r}\right)}\right), \\
\mathbb{P}_{q, \text { old }}^{1}[\Phi ; r] & =\frac{2}{q \log \left(q^{r}\right)} \sum_{\substack{\ell \mid q^{\infty} \\
\ell}} \frac{1}{\substack{p \in \mathcal{P} \\
p \nmid q}} \Delta_{1}\left(p^{r} \ell^{2}, 1\right) \frac{\log p}{\sqrt{p}} \widehat{\Phi}\left(\frac{\log p}{\log \left(q^{r}\right)}\right) .
\end{aligned}
$$

Let us estimate the new part which can be written as

$$
\begin{array}{r}
\mathbb{P}_{q, \text { new }}^{1}[\Phi ; r]=-\frac{2\left(2 \pi i^{\kappa}\right)}{\log \left(q^{r}\right)} \sum_{\substack{c \geqslant 1 \\
q \mid c}} \sum_{\substack{ \\
\text { p }}}\left(\frac{\log p}{\sqrt{p}} \delta_{q \nmid p} 1_{\left[1, q^{r \nu}\right]}(p)\right) \frac{S\left(p^{r}, 1 ; c\right)}{c} \\
\times J_{\kappa-1}\left(\frac{4 \pi \sqrt{p^{r}}}{c}\right) \widehat{\Phi}\left(\frac{\log p}{\log \left(q^{r}\right)}\right) .
\end{array}
$$

Thanks to (A.3), the function

$$
h(m ; c):=J_{\kappa-1}\left(\frac{4 \pi \sqrt{m}}{c}\right)
$$

satisfies hypothesis $\mathrm{P}(T ; M, 1, C)$ with

$$
T(M, 1, C)=\left(1+\frac{\sqrt{M}}{C}\right)^{1 / 2-\kappa}\left(\frac{\sqrt{M}}{C}\right)^{\kappa-1} .
$$


Hence, if $\nu \leqslant 2 / r^{2}$ then corollary 3.5 leads to

$$
\begin{aligned}
\mathbb{P}_{q, \text { new }}^{1}[\Phi ; r] & \ll_{\varepsilon} q^{\varepsilon} \sum_{\substack{1 \leqslant M \leqslant q^{\nu r^{2}} \\
C \geqslant q / 2}}^{\sharp}\left(1+\sqrt{\frac{M}{q}}\right)\left(\frac{\sqrt{M}}{C}\right)^{\kappa-1-2 \theta} \\
& \ll_{\varepsilon} q^{\varepsilon} \sum_{1 \leqslant M \leqslant q^{\nu r^{2}}}^{\sharp}\left(\frac{M^{\frac{\kappa-1}{2}-\theta}}{q^{\kappa-1-2 \theta}}+\frac{M^{\frac{\kappa}{2}-\theta}}{q^{\kappa-\frac{1}{2}-2 \theta}}\right)
\end{aligned}
$$

thanks to (A.2). Summing over $M$ via (A.1) leads to

$$
\mathbb{P}_{q, \text { new }}^{1}[\Phi ; r] \ll_{\varepsilon} q^{\left(\frac{\kappa-1}{2}-\theta\right)\left(r^{2} \nu-2\right)+\varepsilon}+q^{\left(\frac{\kappa}{2}-\theta\right) r^{2} \nu-\left(\kappa-\frac{1}{2}-2 \theta\right)+\varepsilon}
$$

which is an admissible error term if $\nu<\nu_{1, \max }(r, \kappa, \theta)$. According to lemma 3.13 (with $\alpha_{2}=+\infty$ ) we have

$$
\mathbb{P}_{q, \text { old }}^{1}[\Phi ; r] \ll_{\varepsilon} q^{\frac{r \nu}{2}-1+\varepsilon}
$$

which is an admissible error term if $\nu<2 / r$. Reporting (4.7) and (4.8) in (4.4) we obtain

$$
\mathbb{E}_{q}^{\mathrm{h}}\left(P_{q}^{1}[\Phi ; r]\right) \ll \frac{1}{q^{\delta_{1}}}
$$

for some $\delta_{1}>0$ (depending on $\nu$ and $r$ ) as soon as $\nu<\nu_{1, \max }(r, \kappa, \theta)$. We now estimate the third term of (4.3). If $0 \leqslant m \leqslant r-1$ then the trace formula given in proposition 2.2 implies that

$$
\mathbb{E}_{q}^{\mathrm{h}}\left(P_{q}^{2}[\Phi ; r, m]\right)=\mathbb{P}_{q, \text { new }}^{2}[\Phi ; r, m]+\mathbb{P}_{q, \text { old }}^{2}[\Phi ; r, m]
$$

where

$$
\begin{aligned}
& \mathbb{P}_{q, \text { new }}^{2}[\Phi ; r, m]=-\frac{2}{\log \left(q^{r}\right)} \sum_{\substack{p \in \mathcal{P} \\
p \nmid q}} \Delta_{q}\left(p^{2(r-m)}, 1\right) \frac{\log p}{p} \widehat{\Phi}\left(\frac{\log \left(p^{2}\right)}{\log \left(q^{r}\right)}\right), \\
& \mathbb{P}_{q, \text { old }}^{2}[\Phi ; r, m]=\frac{2}{q \log \left(q^{r}\right)} \sum_{\substack { \ell q^{\infty} \\
\begin{subarray}{c}{\ell \\
\ell{ \ell q ^ { \infty } \\
\begin{subarray} { c } { \ell \\
\ell } }\end{subarray}} \frac{1}{\substack{p \in \mathcal{P} \\
p \nmid q}} \Delta_{1}\left(p^{2(r-m)} \ell^{2}, 1\right) \frac{\log p}{p} \widehat{\Phi}\left(\frac{\log \left(p^{2}\right)}{\log \left(q^{r}\right)}\right) .
\end{aligned}
$$

Let us estimate the new part which can be written as

$$
\begin{aligned}
\mathbb{P}_{q, \text { new }}^{2}[\Phi ; r, m]=-\frac{2\left(2 \pi i^{\kappa}\right)}{\log \left(q^{r}\right)} \sum_{\substack{c \geqslant 1 \\
q \mid c}} \sum_{p \in \mathcal{P}}\left(\frac{\log p}{\sqrt{p}} \delta_{q \nmid p} 1_{\left[1, q^{\frac{r \nu}{2}}\right]}(p)\right) \frac{S\left(p^{2(r-m)}, 1 ; c\right)}{c} \\
\times \frac{1}{\sqrt{p}} J_{\kappa-1}\left(\frac{4 \pi \sqrt{p^{2(r-m)}}}{c}\right) \widehat{\Phi}\left(\frac{\log p}{\log q^{r / 2}}\right) .
\end{aligned}
$$

The function

$$
h(m, c):=J_{\kappa-1}\left(\frac{4 \pi \sqrt{m}}{c}\right) \times \frac{1}{m^{1 /(4(r-m))}}
$$

satisfies hypothesis $\mathrm{P}(T ; M, 1, C)$ with

$$
T(M, 1, C)=\left(1+\frac{\sqrt{M}}{C}\right)^{1 / 2-\kappa}\left(\frac{\sqrt{M}}{C}\right)^{\kappa-1} \frac{1}{M^{1 /(4(r-m))}} .
$$


Hence, if $\nu \leqslant 2 / r^{2}$ then corollary 3.5 leads to

$$
\mathbb{P}_{q, \text { new }}^{2}[\Phi ; r, m] \ll_{\varepsilon} q^{\varepsilon} \sum_{\substack{M \leqslant q^{\nu r(r-m)} \\ C \geqslant q / 2}}^{\sharp} \frac{1}{(M)^{1 /(4 r-4 m)}}\left(\frac{\sqrt{M}}{C}\right)^{\kappa-1-2 \theta}\left(1+\sqrt{\frac{M}{q}}\right) .
$$

This is smaller than the bound given in (4.5) and hence is an admissible error term if $\nu<\nu_{1, \max }(r, \kappa, \theta)$. According to lemma 3.13, we have

$$
\mathbb{P}_{q, \text { old }}^{2}[\Phi ; r] \ll{ }_{\varepsilon} q^{-1+\varepsilon} .
$$

We obtain

$$
\mathbb{E}_{q}^{\mathrm{h}}\left(P_{q}^{2}[\Phi ; r, m]\right) \ll \frac{1}{q^{\delta_{2}}}
$$

for some $\delta_{2}>0$ (depending on $\nu$ and $r$ ) as soon as $\nu<\nu_{1, \max }(r, \kappa, \theta)$. Finally, reporting (4.12) and (4.9) in (4.3), we get

$$
\mathbb{E}_{q}^{\mathrm{h}}\left(D_{1, q}[\Phi ; r]\right)=E[\Phi ; r]+O\left(\frac{1}{\log q}\right) .
$$

4.3. Signed asymptotic expectation of the one-level density. In this part, we prove some density results for subfamilies of $\mathcal{F}_{r}$ on which the sign of the functional equation remains constant. The two subfamilies are defined by

$$
\mathcal{F}_{r}^{\varepsilon}:=\bigcup_{q \in \mathcal{P}}\left\{L\left(\operatorname{Sym}^{r} f, s\right), f \in H_{\kappa}^{\varepsilon}(q)\right\} .
$$

Indeed, we compute the asymptotic expectation $\mathbb{E}_{\infty}^{\mathrm{h}, \varepsilon}\left(D_{1}[\Phi ; r]\right)$.

Theorem 4.4- Let $r \geqslant 1$ be an odd integer, $\varepsilon= \pm 1$ and $\Phi \in \mathcal{S}_{\nu}(\mathbb{R})$. We assume that hypothesis $\operatorname{Nice}(r, f)$ holds for any prime number $q$ and any $f \in H_{\kappa}^{*}(q)$ and also that $\theta$ is admissible. Let

$$
\nu_{1, \max }^{\varepsilon}(r, \kappa, \theta):=\inf \left(\nu_{1, \max }(r, \kappa, \theta), \frac{3}{r(r+2)}\right) .
$$

If $\nu<\nu_{1, \max }^{\varepsilon}(r, \kappa, \theta)$ then

$$
\mathbb{E}_{\infty}^{\mathrm{h}, \varepsilon}\left(D_{1}[\Phi ; r]\right)=E[\Phi ; r] .
$$

Some comments are given in remark 5 page 5 .

Proof of theorem 4.4. By (2.18), we have

$$
\mathbb{E}_{q}^{\mathrm{h}, \varepsilon}\left(D_{1, q}[\Phi ; r]\right)=\mathbb{E}_{q}^{\mathrm{h}}\left(D_{1, q}[\Phi ; r]\right)-\varepsilon \times \varepsilon(k, r) \sqrt{q} \mathbb{E}_{q}^{\mathrm{h}}\left(\lambda .(q) D_{1, q}[\Phi ; r]\right) .
$$

The first term is the main term of the theorem thanks to theorem 4.1. According to proposition 3.8 and corollary 3.11, the second term (without the epsilon factors) is given by

$$
\begin{aligned}
\sqrt{q} \mathbb{E}_{q}^{\mathrm{h}}\left(\lambda .(q) P_{q}^{1}[\Phi ; r]\right) & \\
& +\sqrt{q} \sum_{m=0}^{r-1}(-1)^{m} \mathbb{E}_{q}^{\mathrm{h}}\left(\lambda .(q) P_{q}^{2}[\Phi ; r, m]\right)+O\left(\frac{1}{\log \left(q^{r}\right)}\right) .
\end{aligned}
$$


Let us focus on the first term in (4.15) knowing that the same discussion holds for the second term with even better results on $\nu$. We have

$$
\sqrt{q} \mathbb{E}_{q}^{\mathrm{h}}\left(\lambda .(q) P_{q}^{1}[\Phi ; r]\right)=\sqrt{q} \mathbb{P}_{q, \text { new }}^{1}[\Phi ; r]+\sqrt{q} \mathbb{P}_{q, \text { old }}^{1}[\Phi ; r]
$$

where

$$
\begin{aligned}
& \mathbb{P}_{q, \text { new }}^{1}[\Phi ; r]=-\frac{2}{\log \left(q^{r}\right)} \sum_{\substack{p \in \mathcal{P} \\
p \nmid q}} \Delta_{q}\left(p^{r} q, 1\right) \frac{\log p}{\sqrt{p}} \widehat{\Phi}\left(\frac{\log p}{\log \left(q^{r}\right)}\right), \\
& \mathbb{P}_{q, \text { old }}^{1}[\Phi ; r]=\frac{2}{q \nu(q) \log \left(q^{r}\right)} \sum_{\substack{\ell q^{\infty} \\
\ell}} \frac{1}{\substack{p \in \mathcal{P} \\
p \nmid q}} \Delta_{1}\left(p^{r} \ell^{2}, q\right) \frac{\log p}{\sqrt{p}} \widehat{\Phi}\left(\frac{\log p}{\log \left(q^{r}\right)}\right) .
\end{aligned}
$$

Lemma 3.13 implies

$$
\sqrt{q} \mathbb{P}_{q, \text { old }}^{1}[\Phi ; r] \ll q^{(\nu r-4) / 2}
$$

which is an admissible error term if $\nu<4 / r$. The new part is given by

$$
\mathbb{P}_{q, \text { new }}^{1}[\Phi ; r]=-\frac{2\left(2 \pi i^{\kappa}\right)}{\log \left(q^{r}\right)} \sum_{\substack{c \geqslant 1 \\ q \mid c}} \sum_{\substack{p \in \mathcal{P} \\ q \nmid p}} \frac{\log p}{\sqrt{p}} \frac{S\left(p^{r} q, 1 ; c\right)}{c} J_{\kappa-1}\left(\frac{4 \pi \sqrt{p^{r} q}}{c}\right) \widehat{\Phi}\left(\frac{\log (p)}{\log \left(q^{r}\right)}\right) .
$$

and can be written as

$$
-\frac{2\left(2 \pi i^{\kappa}\right)}{\log \left(q^{r}\right)} \sum_{\substack{c \geqslant 1 \\ q \mid c}} \sum_{m \geqslant 1} \widehat{a}_{m} \frac{S(m, 1 ; c)}{c} J_{\kappa-1}\left(\frac{4 \pi \sqrt{m}}{c}\right) \widehat{\Phi}\left(\frac{\log (m / q)}{\log \left(q^{r^{2}}\right)}\right)
$$

where

$$
\widehat{a}_{m}:=1_{\left[1, q^{1+\nu r^{2}}\right]} \begin{cases}0 & \text { if } q \nmid m \text { or } m \neq p^{r} q \text { for some } p \neq q \text { in } \mathcal{P}, \\ \frac{\log p}{\sqrt{p}} & \text { if } m=p^{r} q \text { for some } p \neq q \text { in } \mathcal{P} .\end{cases}
$$

Thus, if $\nu \leqslant 1 / r^{2}$ then we obtain

$$
\mathbb{P}_{q, \text { new }}^{1}[\Phi ; r, m] \ll_{\varepsilon} q^{\varepsilon} \sum_{\substack{M \leqslant q^{1+\nu r^{2}} \\ C \geqslant q / 2}}^{\sharp}\left(\frac{\sqrt{M}}{C}\right)^{\kappa-1-2 \theta}\left(1+\sqrt{\frac{M}{q}}\right)
$$

as in the proof of corollary 3.5. Summing over $C$ via (A.2) gives

$$
\mathbb{P}_{q, \text { new }}^{1}[\Phi ; r, m] \ll_{\varepsilon} q^{\varepsilon} \sum_{M \leqslant q^{1+r^{2} \nu}}^{\sharp}\left(\frac{M^{\frac{\kappa-1}{2}-\theta}}{q^{\kappa-1-2 \theta}}+\frac{M^{\frac{\kappa}{2}-\theta}}{q^{\kappa-\frac{1}{2}-2 \theta}}\right) .
$$

Summing over $M$ via (A.1) leads to

$$
\mathbb{P}_{q, \text { new }}^{1}[\Phi ; r, m] \ll_{\varepsilon} q^{\left(\frac{\kappa-1}{2}-\theta\right) r^{2} \nu-\left(\frac{\kappa-1}{2}-\theta\right)+\varepsilon}+q^{\left(\frac{\kappa}{2}-\theta\right) r^{2} \nu-\left(\frac{\kappa-1}{2}-\theta\right)+\varepsilon}
$$

which is an admissible error term if $\nu<\frac{1}{r^{2}}\left(1-\frac{1}{\kappa-2 \theta}\right)$. 


\section{Quadratic STATistics FOR LOW-LYING ZEROS}

\subsection{Asymptotic expectation of the two-level density and asymp- totic variance.}

Definition 5.1- Let $f \in H_{\kappa}^{*}(q)$ and $\Phi_{1}, \Phi_{2}$ in $\mathcal{S}_{\nu}(\mathbb{R})$. The two-level density (relatively to $\Phi_{1}$ and $\Phi_{2}$ ) of $\operatorname{Sym}^{r} f$ is

$$
D_{2, q}\left[\Phi_{1}, \Phi_{2} ; r\right](f):=\sum_{\substack{\left(j_{1}, j_{2}\right) \in \mathcal{E}(f, r)^{2} \\ j_{1} \neq \pm j_{2}}} \Phi_{1}\left(\widehat{\rho}_{f, r}^{\left(j_{1}\right)}\right) \Phi_{2}\left(\widehat{\rho}_{f, r}^{\left(j_{2}\right)}\right) .
$$

Remark 5.2- In this definition, it is important to note that the condition $j_{1} \neq j_{2}$ does not imply that $\widehat{\rho}_{f, r}^{\left(j_{1}\right)} \neq \widehat{\rho}_{f, r}^{\left(j_{2}\right)}$. It only implies this if the zeros are simple. Recall however that some $L$-functions of elliptic curves (hence of modular forms) have multiple zeros at the critical point $[3,24]$.

The following lemma is an immediate consequence of definition 5.1.

Lemma 5.3- Let $f \in H_{\kappa}^{*}(q)$ and $\Phi_{1}, \Phi_{2}$ in $\mathcal{S}_{\nu}(\mathbb{R})$. Then,

$$
\begin{array}{r}
D_{2, q}\left[\Phi_{1}, \Phi_{2} ; r\right](f)=D_{1, q}\left[\Phi_{1} ; r\right](f) D_{1, q}\left[\Phi_{2} ; r\right] \\
(f)-2 D_{1, q}\left[\Phi_{1} \Phi_{2} ; r\right](f) \\
+1_{H_{\kappa}^{-1}(q)}(f) \times \Phi_{1}(0) \Phi_{2}(0) .
\end{array}
$$

We first evaluate the product of one-level statistics on average.

Lemma 5.4-Let $r \geqslant 1$. Let $\Phi_{1}$ and $\Phi_{2}$ in $\mathcal{S}_{\nu}(\mathbb{R})$. We assume that hypothesis $\operatorname{Nice}(r, f)$ holds for any prime number $q$ and any $f \in H_{\kappa}^{*}(q)$ and also that $\theta$ is admissible. If $\nu<1 / r^{2}$ then

$$
\mathbb{E}_{\infty}^{\mathrm{h}}\left(D_{1}\left[\Phi_{1} ; r\right] D_{1}\left[\Phi_{2} ; r\right]\right)=E\left[\Phi_{1} ; r\right] E\left[\Phi_{2} ; r\right]+2 \int_{\mathbb{R}}|u| \widehat{\Phi_{1}}(u) \widehat{\Phi_{2}}(u) \mathrm{d} u .
$$

Remark 5.5- Since theorem 4.1 implies that

$$
\begin{aligned}
\mathbb{E}_{\infty}^{\mathrm{h}}\left(D_{1}\left[\Phi_{1} ; r\right] D_{1}\left[\Phi_{2} ; r\right]\right)-E\left[\Phi_{1} ; r\right] E\left[\Phi_{2} ; r\right]= \\
\mathbb{E}_{\infty}^{\mathrm{h}}\left(D_{1}\left[\Phi_{1} ; r\right] D_{1}\left[\Phi_{2} ; r\right]\right)-\mathbb{E}_{\infty}^{\mathrm{h}}\left(D_{1}\left[\Phi_{1} ; r\right]\right) \mathbb{E}_{\infty}^{\mathrm{h}}\left(D_{1}\left[\Phi_{2} ; r\right]\right),
\end{aligned}
$$

lemma 5.4 reveals that the term

$$
\mathbb{C}_{\infty}^{\mathrm{h}}\left(D_{1}\left[\Phi_{1} ; r\right], D_{1}\left[\Phi_{2} ; r\right]\right):=2 \int_{\mathbb{R}}|u| \widehat{\Phi_{1}}(u) \widehat{\Phi_{2}}(u) \mathrm{d} u
$$

measures the dependence between $D_{1}\left[\Phi_{1} ; r\right]$ and $D_{1}\left[\Phi_{2} ; r\right]$. This term is the asymptotic covariance of $D_{1}\left[\Phi_{1} ; r\right]$ and $D_{1}\left[\Phi_{2} ; r\right]$. In particular, taking $\Phi_{1}=\Phi_{2}$, we obtain the asymptotic variance.

Theorem 5.6- Let $\Phi \in \mathcal{S}_{\nu}(\mathbb{R})$. If $\nu<1 / r^{2}$ then the asymptotic variance of the random variable $D_{1, q}[\Phi ; r]$ is

$$
\mathbb{V}_{\infty}^{\mathrm{h}}\left(D_{1}[\Phi ; r]\right)=2 \int_{\mathbb{R}}|u| \widehat{\Phi}^{2}(u) \mathrm{d} u .
$$


Proof of lemma 5.4. From proposition 3.8, we obtain

$$
\begin{gathered}
\mathbb{E}_{q}^{\mathrm{h}}\left(D_{1, q}\left[\Phi_{1} ; r\right] D_{1, q}\left[\Phi_{2} ; r\right]\right)=E\left[\Phi_{1} ; r\right] E\left[\Phi_{2} ; r\right]+\mathbb{C}_{q}^{\mathrm{h}} \\
+\sum_{\substack{(i, j) \in\{1,2\}^{2} \\
i \neq j}} \sum_{m=0}^{r-1}(-1)^{m} \mathbb{E}_{q}^{\mathrm{h}}\left(P_{q}^{1}\left[\Phi_{i} ; r\right] P_{q}^{2}\left[\Phi_{j} ; r, m\right]\right) \\
+\sum_{m_{1}=0}^{r-1} \sum_{m_{2}=0}^{r-1}(-1)^{m_{1}+m_{2}} \mathbb{E}_{q}^{\mathrm{h}}\left(P_{q}^{2}\left[\Phi_{1} ; r, m_{1}\right] P_{q}^{2}\left[\Phi_{2} ; r, m_{2}\right]\right)+O\left(\frac{1}{\log \left(q^{r}\right)}\right)
\end{gathered}
$$

with

$$
\mathbb{C}_{q}^{\mathrm{h}}:=\mathbb{E}_{q}^{\mathrm{h}}\left(P_{q}^{1}\left[\Phi_{1} ; r\right] P_{q}^{1}\left[\Phi_{2} ; r\right]\right) .
$$

The error term is evaluated by use of theorem 4.1 and equations (2.16), (4.9) and (4.12). We first compute $\mathbb{C}_{q}^{\mathrm{h}}$. Using proposition 2.2 , we compute $\mathbb{C}_{q}^{\mathrm{h}}=E^{n}-4 E^{o}$ with

$$
E^{n}:=\frac{4}{\log ^{2}\left(q^{r}\right)} \sum_{\substack{p_{1} \in \mathcal{P} \\ p_{1} \nmid q}} \sum_{\substack{p_{2} \in \mathcal{P} \\ p_{2} \nmid q}} \frac{\log p_{1}}{\sqrt{p_{1}}} \frac{\log p_{2}}{\sqrt{p_{2}}} \widehat{\Phi_{1}}\left(\frac{\log p_{1}}{\log \left(q^{r}\right)}\right) \widehat{\Phi_{2}}\left(\frac{\log p_{2}}{\log \left(q^{r}\right)}\right) \Delta_{q}\left(p_{1}^{r}, p_{2}^{r}\right)
$$

and

$$
\begin{aligned}
& E^{o}:=\frac{1}{q \log ^{2}\left(q^{r}\right)} \\
& \quad \times \sum_{\substack{p_{1} \in \mathcal{P} \\
p_{1} \nmid q}} \sum_{\substack{p_{2} \in \mathcal{P} \\
p_{2} \nmid q}} \frac{\log p_{1}}{\sqrt{p_{1}}} \frac{\log p_{2}}{\sqrt{p_{2}}} \widehat{\Phi_{1}}\left(\frac{\log p_{1}}{\log \left(q^{r}\right)}\right) \widehat{\Phi_{2}}\left(\frac{\log p_{2}}{\log \left(q^{r}\right)}\right) \sum_{\ell \mid q^{\infty}} \frac{\Delta_{1}\left(\ell^{2} p_{1}^{r}, p_{2}^{r}\right)}{\ell} .
\end{aligned}
$$

By definition of the $\Delta$-symbol, we write $E^{n}=E_{\mathrm{p}}^{n}+\frac{8 \pi i^{\kappa}}{\log ^{2}\left(q^{r}\right)} E_{\mathrm{e}}^{n}$ with

$$
E_{\mathrm{p}}^{n}:=\frac{4}{\log ^{2}\left(q^{r}\right)} \sum_{\substack{p \in \mathcal{P} \\ p \nmid q}} \frac{\log ^{2} p}{p}\left(\widehat{\Phi_{1}} \widehat{\Phi_{2}}\right)\left(\frac{\log p}{\log \left(q^{r}\right)}\right)
$$

and

$$
\begin{aligned}
E_{\mathrm{e}}^{n}:=\sum_{\substack{c \geqslant 1 \\
q \mid c}} \sum_{\substack{p_{1} \in \mathcal{P} \\
p_{1} \nmid q}} \sum_{p_{2} \in \mathcal{P}} \frac{\log p_{1}}{\sqrt{p_{1}}} \frac{\log p_{2}}{\sqrt{p_{2}}} \widehat{\Phi_{1}}\left(\frac{\log p_{1}}{\log \left(q^{r}\right)}\right) \widehat{\Phi_{2}}\left(\frac{\log p_{2}}{\log \left(q^{r}\right)}\right) \\
\times \frac{S\left(p_{1}^{r}, p_{2}^{r} ; c\right)}{c} J_{\kappa-1}\left(\frac{4 \pi \sqrt{p_{1}^{r} p_{2}^{r}}}{c}\right) .
\end{aligned}
$$

We remove the condition $p \nmid q$ from $E_{\mathrm{p}}^{n}$ at an admissible cost and obtain, after integration by parts,

$$
E_{\mathrm{p}}^{n}=2 \int_{\mathbb{R}}|u| \widehat{\Phi_{1}}(u) \widehat{\Phi_{2}}(u) \mathrm{d} u+O\left(\frac{1}{\log ^{2}\left(q^{r}\right)}\right) .
$$

Using corollary 3.5, we get

$$
E_{\mathrm{e}}^{n} \ll \frac{1}{\log ^{2}\left(q^{r}\right)}
$$


as soon as $\nu \leqslant 1 / r^{2}$. Finally, using lemma 3.13, we see that $E^{o}$ is an admissible error term for $\nu<1 / r$ so that equations (5.2) and (5.3) lead to

$$
\mathbb{C}_{q}^{\mathrm{h}}=2 \int_{\mathbb{R}}|u| \widehat{\Phi_{1}}(u) \widehat{\Phi_{2}}(u) \mathrm{d} u+O\left(\frac{1}{\log ^{2}\left(q^{r}\right)}\right) .
$$

Let $\{i, j\}=\{1,2\}$. We prove next that each $\mathbb{E}_{q}^{\mathrm{h}}\left(P_{q}^{1}\left[\Phi_{i} ; r\right] P_{q}^{2}\left[\Phi_{j} ; r, m\right]\right)$ is an error term when $\nu<1 / r^{2}$. Using proposition 2.2 and lemma 3.13 we have

$$
\begin{aligned}
& \mathbb{E}_{q}^{\mathrm{h}}\left(P_{q}^{1}\left[\Phi_{i} ; r\right] P_{q}^{2}\left[\Phi_{j} ; r, m\right]\right)=\frac{8 \pi i^{\kappa}}{\log ^{2}\left(q^{r}\right)} \sum_{\substack{c \geqslant 1 \\
q \mid c}} \sum_{\substack{p_{1} \in \mathcal{P} \\
p_{1} \nmid q}} \sum_{p_{2} \in \mathcal{P}} \frac{\log p_{1} \nmid q}{\sqrt{p_{1}}} \frac{\log p_{2}}{p_{2}} \widehat{\Phi}_{i}\left(\frac{\log p_{1}}{\log \left(q^{r}\right)}\right) \\
\times & \widehat{\Phi_{j}}\left(\frac{\log p_{2}}{\log \left(q^{r / 2}\right)}\right) \frac{S\left(p_{1}^{r}, p_{2}^{2 r-2 m} ; c\right)}{c} J_{\kappa-1}\left(\frac{4 \pi \sqrt{p_{1}^{r} p_{2}^{2 r-2 m}}}{c}\right)+O\left(\frac{1}{\log \left(q^{r}\right)}\right)^{2} .
\end{aligned}
$$

We use corollary 3.5 to conclude that

$$
\mathbb{E}_{q}^{\mathrm{h}}\left(P_{q}^{1}\left[\Phi_{i} ; r\right] P_{q}^{2}\left[\Phi_{j} ; r, m\right]\right) \ll \frac{1}{\log q}
$$

when $\nu<1 / r^{2}$. Finally, $\mathbb{E}_{q}^{\mathrm{h}}\left(P_{q}^{2}\left[\Phi_{1} ; r, m_{1}\right] P_{q}^{2}\left[\Phi_{2} ; r, m_{2}\right]\right)$ is shown to be an error term in the same way.

Using lemmas 5.3 and 5.4, theorem 4.1, hypothesis Nice $(r, f)$ and remark 3.12 , we prove the following theorem.

Theorem 5.7- Let $r \geqslant 1$. Let $\Phi_{1}$ and $\Phi_{2}$ in $\mathcal{S}_{\nu}(\mathbb{R})$. We assume that hypothesis Nice $(r, f)$ holds for any prime number $q$ and any $f \in H_{\kappa}^{*}(q)$ and also that $\theta$ is admissible. If $\nu<\nu_{2, \max }(r, \kappa, \theta)$ then

$$
\begin{aligned}
& \mathbb{E}_{\infty}^{\mathrm{h}}\left(D_{2}\left[\Phi_{1}, \Phi_{2} ; r\right]\right)=\left[\widehat{\Phi_{1}}(0)+\frac{(-1)^{r+1}}{2} \Phi_{1}(0)\right]\left[\widehat{\Phi_{2}}(0)+\frac{(-1)^{r+1}}{2} \Phi_{2}(0)\right] \\
& +2 \int_{\mathbb{R}}|u| \widehat{\Phi_{1}}(u) \widehat{\Phi_{2}}(u) \mathrm{d} u-2 \widehat{\Phi_{1} \Phi_{2}}(0)+\left((-1)^{r}+\frac{1_{2 \mathbb{N}+1}(r)}{2}\right) \Phi_{1}(0) \Phi_{2}(0) .
\end{aligned}
$$

Some comments are given in remark 6 page 7 .

5.2. Signed asymptotic expectation of the two-level density and signed asymptotic variance. In this part, $r$ is odd.

Lemma 5.8- Let $\Phi_{1}$ and $\Phi_{2}$ in $\mathcal{S}_{\nu}(\mathbb{R})$. If $\nu<1 /\left(2 r^{2}\right)$ then

$$
\mathbb{E}_{\infty}^{\mathrm{h}, \varepsilon}\left(D_{1}\left[\Phi_{1} ; r\right] D_{1}\left[\Phi_{2} ; r\right]\right)=E\left[\Phi_{1} ; r\right] E\left[\Phi_{2} ; r\right]+2 \int_{\mathbb{R}}|u| \widehat{\Phi_{1}}(u) \widehat{\Phi_{2}}(u) \mathrm{d} u .
$$

Remark 5.9- By theorem 4.4 and lemma 5.8 we have

$$
\begin{aligned}
& \mathbb{E}_{\infty}^{\mathrm{h}, \varepsilon}\left(D_{1}\left[\Phi_{1} ; r\right] D_{1}\left[\Phi_{2} ; r\right]\right)-E\left[\Phi_{1} ; r\right] E\left[\Phi_{2} ; r\right]= \\
& \mathbb{E}_{\infty}^{\mathrm{h}, \varepsilon}\left(D_{1}\left[\Phi_{1} ; r\right] D_{1}\left[\Phi_{2} ; r\right]\right)-\mathbb{E}_{\infty}^{\mathrm{h}, \varepsilon}\left(D_{1}\left[\Phi_{1} ; r\right]\right) \mathbb{E}_{\infty}^{\mathrm{h}, \varepsilon}\left(D_{1}\left[\Phi_{2} ; r\right]\right) .
\end{aligned}
$$

Thus,

$$
\mathbb{C}_{\infty}^{\mathrm{h}, \varepsilon}\left(D_{1}\left[\Phi_{1} ; r\right], D_{1}\left[\Phi_{2} ; r\right]\right):=2 \int_{\mathbb{R}}|u| \widehat{\Phi_{1}}(u) \widehat{\Phi_{2}}(u) \mathrm{d} u
$$


is the signed asymptotic covariance of $D_{1}\left[\Phi_{1} ; r\right]$ and $D_{1}\left[\Phi_{2} ; r\right]$. In particular, taking $\Phi_{1}=\Phi_{2}$, we obtain the signed asymptotic variance.

Theorem 5.10- Let $\Phi \in \mathcal{S}_{\nu}(\mathbb{R})$. If $\nu<1 /\left(2 r^{2}\right)$ then the signed asymptotic variance of $D_{1}[\Phi ; r]$ is

$$
\mathbb{V}_{\infty}^{\mathrm{h}, \varepsilon}\left(D_{1}[\Phi ; r]\right)=2 \int_{\mathbb{R}}|u| \widehat{\Phi}^{2}(u) \mathrm{d} u .
$$

Proof of lemma 5.8. From proposition 3.8 and (3.21), we obtain

$$
\begin{gathered}
\mathbb{E}_{q}^{\mathrm{h}, \varepsilon}\left(D_{1, q}\left[\Phi_{1} ; r\right] D_{1, q}\left[\Phi_{2} ; r\right]\right)=E\left[\Phi_{1} ; r\right] E\left[\Phi_{2} ; r\right]+\mathbb{C}_{q}^{\mathrm{h}, \varepsilon} \\
+\sum_{\substack{(i, j) \in\{1,2\}^{2} \\
i \neq j}} \sum_{m=0}^{r-1}(-1)^{m} \mathbb{E}_{q}^{\mathrm{h}, \varepsilon}\left(P_{q}^{1}\left[\Phi_{i} ; r\right] P_{q}^{2}\left[\Phi_{j} ; r, m\right]\right) \\
+\sum_{m_{1}=0}^{r-1} \sum_{m_{2}=0}^{r-1}(-1)^{m_{1}+m_{2}} \mathbb{E}_{q}^{\mathrm{h}, \varepsilon}\left(P_{q}^{2}\left[\Phi_{1} ; r, m_{1}\right] P_{q}^{2}\left[\Phi_{2} ; r, m_{2}\right]\right)+O\left(\frac{1}{\log \left(q^{r}\right)}\right)
\end{gathered}
$$

with

$$
\mathbb{C}_{q}^{\mathrm{h}, \varepsilon}:=\mathbb{E}_{q}^{\mathrm{h}, \varepsilon}\left(P_{q}^{1}\left[\Phi_{1} ; r\right] P_{q}^{1}\left[\Phi_{2} ; r\right]\right) .
$$

Assume that $\nu<1 / r^{2}$. Then equations (2.18), (5.4) and proposition 2.2 lead to

$$
\mathbb{C}_{q}^{\mathrm{h}, \varepsilon}=2 \int_{\mathbb{R}}|u| \widehat{\Phi_{1}}(u) \widehat{\Phi_{2}}(u) \mathrm{d} u-\varepsilon \times \varepsilon(\kappa, r)\left(G^{n}-4 G^{o}\right)
$$

with

$$
G^{n}:=\frac{4 \sqrt{q}}{\log ^{2}\left(q^{r}\right)} \sum_{\substack{p_{1} \in \mathcal{P} \\ p_{1} \nmid q}} \sum_{p_{2} \in \mathcal{P}} \frac{\log p_{1}}{\sqrt{p_{1}}} \frac{\log p_{2}}{\sqrt{p_{2}}} \widehat{\Phi_{1}}\left(\frac{\log p_{1}}{\log \left(q^{r}\right)}\right) \widehat{\Phi_{2}}\left(\frac{\log p_{2}}{\log \left(q^{r}\right)}\right) \Delta_{q}\left(p_{1}^{r} q, p_{2}^{r}\right)
$$

and

$$
\begin{aligned}
& G^{o}:=\frac{1}{\sqrt{q} \log ^{2}\left(q^{r}\right)} \\
& \times \sum_{\substack{p_{1} \in \mathcal{P} \\
p_{1} \nmid q}} \sum_{\substack{p_{2} \in \mathcal{P} \\
p_{2} \nmid q}} \frac{\log p_{1}}{\sqrt{p_{1}}} \frac{\log p_{2}}{\sqrt{p_{2}}} \widehat{\Phi_{1}}\left(\frac{\log p_{1}}{\log \left(q^{r}\right)}\right) \widehat{\Phi_{2}}\left(\frac{\log p_{2}}{\log \left(q^{r}\right)}\right) \sum_{\ell \mid q^{\infty}} \frac{\Delta_{q}\left(\ell^{2} p_{1}^{r}, p_{2}^{r} q\right)}{\ell} .
\end{aligned}
$$

Lemma 3.10 implies that if $\nu<1 /\left(2 r^{2}\right)$ then

$$
G^{n} \ll \frac{q^{\nu r[r(\kappa-1)+1] / 2}}{q^{(\kappa-1) / 2}}
$$

hence $G^{n}$ is an error term as soon as $\nu \leqslant 1 /\left(2 r^{2}\right)$. Lemma 3.13 implies

$$
G^{o} \ll q^{-3 / 2+\nu r+\varepsilon}
$$

which is an error term. Reporting equations (5.9) and (5.10) in (5.7) we obtain

$$
\mathbb{C}_{\infty}^{\mathrm{h}, \varepsilon}=2 \int_{\mathbb{R}}|u| \widehat{\Phi_{1}}(u) \widehat{\Phi_{2}}(u) \mathrm{d} u
$$


for $\nu \leqslant 1 /(2 r(r+2))$. Next, we prove that each $\mathbb{E}_{q}^{\mathrm{h}, \varepsilon}\left(P_{q}^{1}\left[\Phi_{i} ; r\right] P_{q}^{2}\left[\Phi_{j} ; r, m\right]\right)$ is an error term as soon as $\nu \leqslant 1 /\left(2 r^{2}\right)$. From equations (2.18) and (5.5), we obtain

$$
\begin{aligned}
& \mathbb{E}_{q}^{\mathrm{h}, \varepsilon}\left(P_{q}^{1}\left[\Phi_{i} ; r\right] P_{q}^{2}\left[\Phi_{j} ; r, m\right]\right)= \\
& -\varepsilon \times \varepsilon(\kappa, r) \sqrt{q} \sum_{f \in H_{\kappa}^{*}(q)}^{\mathrm{h}} \lambda_{f}(q) P_{q}^{1}\left[\Phi_{i} ; r\right] P_{q}^{2}\left[\Phi_{j} ; r, m\right]+O\left(\frac{1}{\log q)}\right) .
\end{aligned}
$$

We use proposition 2.2 and lemmas 3.13 and 3.10 to have

$$
\begin{aligned}
& \sqrt{q} \sum_{f \in H_{\kappa}^{*}(q)}^{\mathrm{h}} \lambda_{f}(q) P_{q}^{1}\left[\Phi_{i} ; r\right] P_{q}^{2}\left[\Phi_{j} ; r, m\right] \ll \\
& \frac{q^{\nu r(2 r-m+2) / 4-1 / 4}}{\log ^{2} q}+\frac{q^{(\nu r-1) / 2+\varepsilon}}{\log q} .
\end{aligned}
$$

It follows from (5.13) and (5.12) that

$$
\mathbb{E}_{\infty}^{\mathrm{h}, \varepsilon}\left(P_{q}^{1}\left[\Phi_{i} ; r\right] P_{q}^{2}\left[\Phi_{j} ; r, m\right]\right)=0
$$

for $\nu \leqslant 1 /(2 r(r+1))$. In the same way, we have, for $\nu$ in the previous range,

$$
\mathbb{E}_{\infty}^{\mathrm{h}, \varepsilon}\left(P_{q}^{2}\left[\Phi_{1} ; r, m_{1}\right] P_{q}^{2}\left[\Phi_{2} ; r, m_{2}\right]\right)=0 .
$$

Reporting (5.11), (5.14) and (5.15) in (5.6), we have the announced result.

Using lemmas 5.3, 5.8, theorem 4.4, hypothesis Nice $(r, f)$ and (3.21), we prove the following theorem.

Theorem 5.11- Let $f \in H_{\kappa}^{*}(q)$ and $\Phi_{1}, \Phi_{2}$ in $\mathcal{S}_{\nu}(\mathbb{R})$. If $\nu<1 /(2 r(r+1))$ then

$$
\begin{aligned}
& \mathbb{E}_{\infty}^{\mathrm{h}, \varepsilon}\left(D_{2}\left[\Phi_{1}, \Phi_{2} ; r\right]\right)=\left[\widehat{\Phi_{1}}(0)+\frac{1}{2} \Phi_{1}(0)\right]\left[\widehat{\Phi_{2}}(0)+\frac{1}{2} \Phi_{2}(0)\right] \\
&+2 \int_{\mathbb{R}}|u| \widehat{\Phi_{1}}(u) \widehat{\Phi_{2}}(u) \mathrm{d} u-2 \widehat{\Phi_{1} \Phi_{2}}(0)- \Phi_{1}(0) \Phi_{2}(0) \\
&+1_{\{-1\}}(\varepsilon) \Phi_{1}(0) \Phi_{2}(0) .
\end{aligned}
$$

Remark 5.12- Remark 4.3 together with theorem 5.11 and a result of Katz \& Sarnak (see [19, Theorem A.D.2.2] or [26, Theorem 3.2]) imply that the symmetry type of $\mathcal{F}_{r}^{\varepsilon}$ is as in table 2 . Some additional comments are given in remark 2 page 3 .

\section{First asymptotic moments of the one-Level Density}

In this section, we compute the asymptotic $m$-th moment of the one level density namely

$$
\mathbb{M}_{\infty, m}^{\mathrm{h}}\left(D_{1, q}[\Phi ; r]\right):=\lim _{\substack{q \in \mathcal{P} \\ q \rightarrow+\infty}} \mathbb{M}_{q, m}^{\mathrm{h}}\left(D_{1, q}[\Phi ; r]\right)
$$

where

$$
\mathbb{M}_{q, m}^{\mathrm{h}}\left(D_{1, q}[\Phi ; r]\right)=\mathbb{E}_{q}^{\mathrm{h}}\left(\left(D_{1, q}[\Phi ; r]-\mathbb{E}_{q}^{\mathrm{h}}\left(D_{1, q}[\Phi ; r]\right)\right)^{m}\right)
$$


for $m$ small enough (regarding to the size of the support of $\Phi$ ). The end of this section is devoted to the proof of theorem E. Note that we can assume that $m \geqslant 3$ since the work has already been done for $m=1$ and $m=2$. Thanks to equation (4.13) and proposition 3.8, we have

$$
\begin{aligned}
\mathbb{M}_{q, m}^{\mathrm{h}}\left(D_{1, q}[\Phi ; r]\right) & =\sum_{\ell=0}^{m}\left(\begin{array}{c}
m \\
\ell
\end{array}\right) \mathbb{E}_{q}^{\mathrm{h}}\left(P_{q}^{1}[\Phi ; r]^{m-\ell}\left(P_{q}^{2}[\Phi ; r]+O\left(\frac{1}{\log q}\right)\right)^{\ell}\right) \\
& =\sum_{\substack{0 \leqslant \ell \leqslant m \\
0 \leqslant \alpha \leqslant \ell}}\left(\begin{array}{c}
m \\
\ell
\end{array}\right)\left(\begin{array}{c}
\ell \\
\alpha
\end{array}\right) R(q)^{\ell-\alpha} \mathbb{E}_{q}^{\mathrm{h}}\left(P_{q}^{1}[\Phi ; r]^{m-\ell} P_{q}^{2}[\Phi ; r]^{\alpha}\right)
\end{aligned}
$$

where

$$
\begin{aligned}
P_{q}^{2}[\Phi ; r](f) & :=-\frac{2}{\log \left(q^{r}\right)} \sum_{j=0}^{r-1}(-1)^{j} \sum_{\substack{p \in \mathcal{P} \\
p \nmid q}} \lambda_{f}\left(p^{2(r-j)}\right) \frac{\log p}{p} \widehat{\Phi}\left(\frac{2 \log p}{\log \left(q^{r}\right)}\right) \\
& =-\frac{2}{\log \left(q^{r}\right)} \sum_{j=1}^{r}(-1)^{r-j} \sum_{\substack{p \in \mathcal{P} \\
p \nmid q}} \lambda_{f}\left(p^{2 j}\right) \frac{\log p}{p} \widehat{\Phi}\left(\frac{2 \log p}{\log \left(q^{r}\right)}\right)
\end{aligned}
$$

and $R$ is a positive function satisfying

$$
R(q) \ll \frac{1}{\log q} .
$$

Thus, an asymptotic formula for $\mathbb{M}_{q, m}^{\mathrm{h}}\left(D_{1, q}[\Phi ; r]\right)$ directly follows from the next proposition.

Proposition 6.1- Let $r \geqslant 1$ be any integer. We assume that hypothesis Nice $(r, f)$ holds for any prime number $q$ and any primitive holomorphic cusp form of level $q$ and even weight $\kappa$. Let $\alpha \geqslant 0$ and $\ell \geqslant 0$ be any integers.

- If $\alpha \geqslant 1$ and $\alpha \nu<4 / r^{2}$ then

$$
\mathbb{E}_{q}^{\mathrm{h}}\left(P_{q}^{2}[\Phi ; r]^{\alpha}\right)=O\left(\frac{1}{\log q}\right) .
$$

- If $1 \leqslant \alpha \leqslant \ell \leqslant m-1$ and $(\alpha+m-\ell) \nu<4 /(r(r+2))$ then

$$
\mathbb{E}_{q}^{\mathrm{h}}\left(P_{q}^{1}[\Phi ; r]^{m-\ell} P_{q}^{2}[\Phi ; r]^{\alpha}\right)=O\left(\frac{1}{\log q}\right) .
$$

- If $\alpha \geqslant 1$ and $\alpha \nu<4 /(r(r+2))$ then

$$
\mathbb{E}_{q}^{\mathrm{h}}\left(P_{q}^{1}[\Phi ; r]^{\alpha}\right)= \begin{cases}O\left(\frac{1}{\log ^{2}(q)}\right) & \text { if } \alpha \text { is odd, } \\ 2 \int_{\mathbb{R}}|u| \widehat{\Phi}^{2}(u) \mathrm{d} u \times \frac{\alpha !}{2^{\alpha / 2}\left(\frac{\alpha}{2}\right) !}+O\left(\frac{1}{\log ^{2}(q)}\right) & \text { otherwise. }\end{cases}
$$


6.1. One some useful combinatorial identity. In order to use the multiplicative properties of Hecke eigenvalues in the proof of proposition 6.1, we want to reorder some sums over many primes to sums over distinct primes. We follow the work of Hughes \& Rudnick [14, §7] (see also [13] and the work of Soshnikov [28]) to achieve this. Let $P(\alpha, s)$ be the set of surjective functions

$$
\sigma:\{1, \ldots, \alpha\} \rightarrow\{1, \ldots, s\}
$$

such that for any $j \in\{1, \ldots, \alpha\}$, either $\sigma(j)=1$ or there exists $k<j$ such that $\sigma(j)=\sigma(k)+1$. This can be viewed as the number of partitions of a set of $\alpha$ elements into $s$ nonempty subsets. By definition, the cardinality of $P(\alpha, s)$ is the Stirling number of second kind [29, §1.4]. For any $j \in$ $\{1, \ldots, s\}$, let

$$
\varpi_{j}^{(\sigma)}:=\# \sigma^{-1}(\{j\})
$$

Note that

$$
\varpi_{j}^{(\sigma)} \geqslant 1 \quad \text { for any } 1 \leqslant j \leqslant s \quad \text { and } \quad \sum_{j=1}^{s} \varpi_{j}^{(\sigma)}=\alpha .
$$

The following lemma is lemma 7.3 of $[14, \S 7]$.

Lemma 6.2- If $g$ is any function of $m$ variables then

$$
\sum_{j_{1}, \ldots, j_{m}} g\left(x_{j_{1}}, \ldots, x_{j_{m}}\right)=\sum_{s=1}^{m} \sum_{\sigma \in P(m, s)} \sum_{\begin{array}{c}
i_{1}, \ldots, i_{s} \\
\text { distinct }
\end{array}} g\left(x_{i_{\sigma(1)}}, \ldots, x_{i_{\sigma(m)}}\right) .
$$

6.2. Proof of the first bullet of proposition 6.1. By the definition (6.5), we have

$$
\begin{aligned}
\mathbb{E}_{q}^{\mathrm{h}}\left(P_{q}^{2}[\Phi ; r]^{\alpha}\right)=\frac{(-2)^{\alpha}}{\log ^{\alpha}\left(q^{r}\right)} \sum_{1 \leqslant j_{1}, \ldots, j_{\alpha} \leqslant r}(-1)^{\alpha r-\left(j_{1}+\ldots+j_{\alpha}\right)} \\
\quad \times \sum_{\substack{p_{1}, \ldots, p_{\alpha} \in \mathcal{P} \\
q \nmid p_{1} \ldots p_{\alpha}}}\left(\prod_{i=1}^{\alpha} \frac{\log p_{i}}{p_{i}} \widehat{\Phi}\left(\frac{2 \log p_{i}}{\log \left(q^{r}\right)}\right)\right) \mathbb{E}_{q}^{\mathrm{h}}\left(\prod_{i=1}^{\alpha} \lambda_{f}\left(p_{i}^{2 j_{i}}\right)\right) .
\end{aligned}
$$

Writing $\left\{\widehat{p}_{i}\right\}_{i \geqslant 1}$ for the increasing sequence of prime numbers except $q$, we have

$$
\begin{aligned}
\sum_{\substack{p_{1}, \ldots, p_{\alpha} \in \mathcal{P} \\
q \nmid p_{1} \ldots p_{\alpha}}}\left(\prod_{i=1}^{\alpha} \frac{\log p_{i}}{p_{i}} \widehat{\Phi}\left(\frac{2 \log p_{i}}{\log \left(q^{r}\right)}\right)\right) \mathbb{E}_{q}^{\mathrm{h}}\left(\prod_{i=1}^{\alpha} \lambda_{f}\left(p_{i}^{2 j_{i}}\right)\right) \\
=\sum_{i_{1}, \ldots, i_{\alpha}}\left(\prod_{\ell=1}^{\alpha} \frac{\log \widehat{p}_{i_{\ell}}}{\widehat{p}_{i_{\ell}}} \widehat{\Phi}\left(\frac{2 \log \widehat{p}_{i_{\ell}}}{\log \left(q^{r}\right)}\right)\right) \mathbb{E}_{q}^{\mathrm{h}}\left(\prod_{\ell=1}^{\alpha} \lambda_{f}\left(\widehat{p}_{i_{\ell}}^{2 j_{\ell}}\right)\right) .
\end{aligned}
$$


Using lemma 6.2, we rewrite the right sum in (6.8) as

$$
\begin{aligned}
& \sum_{s=1}^{\alpha} \sum_{\sigma \in P(\alpha, s)} \sum_{\substack{k_{1}, \ldots, k_{s} \\
\text { distinct }}}\left(\prod_{i=1}^{\alpha} \frac{\log \widehat{p}_{k_{\sigma(i)}}}{\widehat{p}_{k_{\sigma(i)}}} \widehat{\Phi}\left(\frac{2 \log \widehat{p}_{k_{\sigma(i)}}}{\log \left(q^{r}\right)}\right)\right) \mathbb{E}_{q}^{\mathrm{h}}\left(\prod_{i=1}^{\alpha} \lambda_{f}\left(\widehat{p}_{k_{\sigma(i)}}^{2 j_{i}}\right)\right) \\
= & \sum_{s=1}^{\alpha} \sum_{\sigma \in P(\alpha, s)} \sum_{\substack{k_{1}, \ldots, k_{s} \\
\text { distinct }}}\left(\prod_{u=1}^{s}\left(\frac{\log \widehat{p}_{k_{u}}}{\widehat{p}_{k_{u}}} \widehat{\Phi}\left(\frac{2 \log \widehat{p}_{k_{u}}}{\log \left(q^{r}\right)}\right)\right)^{\varpi_{u}^{(\sigma)}}\right) \mathbb{E}_{q}^{\mathrm{h}}\left(\prod_{\substack{1 \leqslant u \leqslant s \\
1 \leqslant j \leqslant r}} \lambda_{f}\left(\widehat{p}_{k_{u}}^{2 j}\right)^{\varpi_{u, j}^{(\sigma)}}\right)
\end{aligned}
$$

where

$$
\varpi_{u, j}^{(\sigma)}:=\#\left\{1 \leqslant i \leqslant \alpha, \sigma(i)=u, j_{i}=j\right\}
$$

for any $1 \leqslant u \leqslant s$ and any $1 \leqslant j \leqslant r$. Now, we show that

$$
\begin{aligned}
& \sum_{s=1}^{\alpha-1} \sum_{\sigma \in P(\alpha, s)} \sum_{\substack{k_{1}, \ldots, k_{s} \\
\text { distinct }}}\left(\prod_{u=1}^{s}\left(\frac{\log \widehat{p}_{k_{u}}}{\widehat{p}_{k_{u}}} \widehat{\Phi}\left(\frac{2 \log \widehat{p}_{k_{u}}}{\log \left(q^{r}\right)}\right)\right)^{\varpi_{u}^{(\sigma)}}\right) \mathbb{E}_{q}^{\mathrm{h}}\left(\prod_{\substack{1 \leqslant u \leqslant s \\
1 \leqslant j \leqslant r}} \lambda_{f}\left(\widehat{p}_{k_{u}}^{2 j}\right)^{\left.\varpi_{u, j}^{(\sigma)}\right)}\right. \\
& \ll \log ^{\alpha-1}(q) . \quad(6.10)
\end{aligned}
$$

For $s<\alpha$ and $\sigma \in P(\alpha, s)$, we use (2.3) together with (3.20) to obtain that the left-hand side of the previous equation is bounded by

$$
\sum_{s=1}^{\alpha-1} \sum_{\sigma \in P(\alpha, s)} \sum_{\substack{k_{1}, \ldots, k_{s} \\ \text { distinct }}} \prod_{u=1}^{s}\left(\frac{\log \widehat{p}_{k_{u}}}{\widehat{p}_{k_{u}}}\left|\widehat{\Phi}\left(\frac{2 \log \widehat{p}_{k_{u}}}{\log \left(q^{r}\right)}\right)\right|\right)^{\varpi_{u}^{(\sigma)}}
$$

Since $s<\alpha$, equation (6.6) implies that $\varpi_{u}^{(\sigma)}>1$ for some $1 \leqslant u \leqslant s$. These values lead to convergent, hence bounded, sums. Let

$$
d^{(\sigma)}:=\#\left\{1 \leqslant u \leqslant s: \varpi_{u}^{(\sigma)}=1\right\} \in\{0, \ldots, \alpha-1\}
$$

then

$$
\begin{aligned}
& \sum_{s=1}^{\alpha-1} \sum_{\sigma \in P(\alpha, s)} \sum_{\substack{k_{1}, \ldots, k_{s} \\
\text { distinct }}} \prod_{u=1}^{s}\left(\frac{\log \widehat{p}_{k_{u}}}{\widehat{p}_{k_{u}}}\left|\widehat{\Phi}\left(\frac{2 \log \widehat{p}_{k_{u}}}{\log \left(q^{r}\right)}\right)\right|\right)^{\varpi_{u}^{(\sigma)}} \\
& \ll \sum_{s=1}^{\alpha-1} \sum_{\sigma \in P(\alpha, s)} \sum_{\substack{k_{1}, \ldots, k_{d} \\
\text { distinct }}} \prod_{u=1}^{d^{(\sigma)}}\left(\frac{\log \widehat{p}_{k_{u}}}{\widehat{p}_{k_{u}}}\left|\widehat{\Phi}\left(\frac{2 \log \widehat{p}_{k_{u}}}{\log \left(q^{r}\right)}\right)\right|\right) \ll \log ^{\alpha-1}(q) .
\end{aligned}
$$


We have altogether

$$
\begin{array}{r}
\mathbb{E}_{q}^{\mathrm{h}}\left(P_{q}^{2}[\Phi ; r]^{\alpha}\right)=\frac{(-2)^{\alpha}}{\log ^{\alpha}\left(q^{r}\right)} \sum_{1 \leqslant j_{1}, \ldots, j_{\alpha} \leqslant r}(-1)^{\alpha r-\left(j_{1}+\ldots+j_{\alpha}\right)} \\
\times \sum_{\substack{k_{1}, \ldots, k_{\alpha} \\
\text { distinct }}}\left(\prod_{u=1}^{\alpha}\left(\frac{\log \widehat{p}_{k_{u}}}{\widehat{p}_{k_{u}}} \widehat{\Phi}\left(\frac{2 \log \widehat{p}_{k_{u}}}{\log \left(q^{r}\right)}\right)\right)\right) \mathbb{E}_{q}^{\mathrm{h}}\left(\lambda_{f}\left(\prod_{u=1}^{\alpha} \widehat{p}_{k_{u}}^{2 j_{u}}\right)\right) \\
+O\left(\frac{1}{\log q}\right)
\end{array}
$$

since the only element of $P(\alpha, \alpha)$ is the identity function. By lemmas 3.9 and 3.10 , we have

$$
\mathbb{E}_{q}^{\mathrm{h}}\left(\lambda_{f}\left(\prod_{u=1}^{\alpha} \widehat{p}_{k_{u}}^{2 j_{u}}\right)\right) \ll \frac{1}{q} \prod_{u=1}^{\alpha} \widehat{p}_{k_{u}}^{j_{u} / 2} \log \widehat{p}_{k_{u}}
$$

hence the first term in the right-hand side of (6.13) is bounded by a negative power of $q$ as soon as $\alpha \nu r^{2}<4$.

6.3. Proof of the third bullet of proposition 6.1. By proposition 3.8, we have

$\mathbb{E}_{q}^{\mathrm{h}}\left(P_{q}^{1}[\Phi ; r]^{\alpha}\right)=\frac{(-2)^{\alpha}}{\log ^{\alpha}\left(q^{r}\right)} \sum_{\substack{p_{1}, \ldots, p_{\alpha} \in \mathcal{P} \\ p_{1}, \ldots, p_{\alpha} \nmid q}}\left(\prod_{i=1}^{\alpha} \frac{\log p_{i}}{\sqrt{p_{i}}} \widehat{\Phi}\left(\frac{\log p_{i}}{\log q^{r}}\right)\right) \mathbb{E}_{q}^{\mathrm{h}}\left(\prod_{i=1}^{\alpha} \lambda_{f}\left(p_{i}^{r}\right)\right)$.

Using lemma 6.2, we rewrite equation (6.14) as

$$
\begin{aligned}
& \mathbb{E}_{q}^{\mathrm{h}}\left(P_{q}^{1}[\Phi ; r]^{\alpha}\right)=\frac{(-2)^{\alpha}}{\log ^{\alpha}\left(q^{r}\right)} \sum_{s=1}^{\alpha} \sum_{\sigma \in P(\alpha, s)} \sum_{\begin{array}{c}
i_{1}, \ldots, i_{s} \\
\text { distinct }
\end{array}}\left(\prod_{j=1}^{\alpha}\left(\frac{\log \widehat{p}_{i_{\sigma(j)}}}{\sqrt{\widehat{p}_{i_{\sigma(j)}}}} \widehat{\Phi}\left(\frac{\log \widehat{p}_{i_{\sigma(j)}}}{\log \left(q^{r}\right)}\right)\right)\right) \\
& \times \mathbb{E}_{q}^{\mathrm{h}}\left(\prod_{j=1}^{\alpha} \lambda_{f}\left(\widehat{p}_{i_{\sigma(j)}}\right)\right) \\
& =\frac{(-2)^{\alpha}}{\log ^{\alpha}\left(q^{r}\right)} \sum_{s=1}^{\alpha} \sum_{\sigma \in P(\alpha, s)} \sum_{\substack{i_{1}, \ldots ., i_{s} \\
\text { distinct }}}\left(\prod_{u=1}^{s}\left(\frac{\log \widehat{p}_{i_{u}}}{\sqrt{\widehat{p}_{i_{u}}}} \widehat{\Phi}\left(\frac{\log \widehat{p}_{i_{u}}}{\log q^{r}}\right)\right)^{\varpi_{u}^{(\sigma)}}\right) \\
& \times \mathbb{E}_{q}^{\mathrm{h}}\left(\prod_{u=1}^{s} \lambda_{f}\left(\widehat{p}_{i_{u}}^{r}\right)^{\varpi_{u}^{(\sigma)}}\right)
\end{aligned}
$$

It follows from (2.11) and (2.12) that

$$
\lambda_{f}\left(\widehat{p}_{i_{u}}^{r}\right)^{\varpi_{u}^{(\sigma)}}=\sum_{j_{u}=0}^{r \varpi_{u}^{(\sigma)}} x\left(\varpi_{u}^{(\sigma)}, r, j_{u}\right) \lambda_{f}\left(\widehat{p}_{i_{u}}^{j_{u}}\right) .
$$


Since $u \neq v$ implies that $\widehat{p}_{i_{u}} \neq \widehat{p}_{i_{v}}$, equation (6.18) becomes

$$
\begin{aligned}
\mathbb{E}_{q}^{\mathrm{h}}\left(P_{q}^{1}[\Phi ; r]^{\alpha}\right)= & \frac{(-2)^{\alpha}}{\log ^{\alpha}\left(q^{r}\right)} \sum_{s=1}^{\alpha} \sum_{\sigma \in P(\alpha, s)} \sum_{\substack{i_{1}, \ldots, i_{s} \\
\text { distinct }}}\left(\prod_{u=1}^{s}\left(\frac{\log \widehat{p}_{i_{u}}}{\sqrt{\widehat{p}_{i_{u}}}} \widehat{\Phi}\left(\frac{\log \widehat{p}_{i_{u}}}{\log \left(q^{r}\right)}\right)\right)^{\varpi_{u}^{(\sigma)}}\right) \\
\times & \sum_{\substack{j_{1}, \ldots, j_{s} \\
0 \leqslant j_{u} \leqslant r \varpi_{u}^{(\sigma)}}}\left(\prod_{u=1}^{s} x\left(\varpi_{u}^{(\sigma)}, r, j_{u}\right)\right) \mathbb{E}_{q}^{\mathrm{h}}\left(\lambda_{f}\left(\prod_{u=1}^{s} \widehat{p}_{i_{u}}^{j_{u}}\right)\right) \cdot(6.19)
\end{aligned}
$$

Using proposition 2.2 and lemmas 3.10 and 3.9 , we get

$$
\mathbb{E}_{q}^{\mathrm{h}}\left(\lambda_{f}\left(\prod_{u=1}^{s} \widehat{p}_{i_{u}}^{j_{u}}\right)\right)=\prod_{u=1}^{s} \delta_{j_{u}, 0}+O\left(\frac{1}{q} \prod_{u=1}^{s} \hat{p}_{i_{u}}^{j_{u} / 4} \log \widehat{p}_{i_{u}}\right)
$$

hence

$$
\mathbb{E}_{q}^{\mathrm{h}}\left(P_{q}^{1}[\Phi ; r]^{\alpha}\right)=\mathrm{TP}+O(\mathrm{TE})
$$

with

$$
\mathrm{TP}:=\frac{(-2)^{\alpha}}{\log ^{\alpha}\left(q^{r}\right)} \sum_{s=1}^{\alpha} \sum_{\sigma \in P(\alpha, s)} \sum_{\begin{array}{c}
i_{1}, \ldots, i_{s} \\
\text { distinct }
\end{array}} \prod_{u=1}^{s}\left(\frac{\log \widehat{p}_{i_{u}}}{\sqrt{\widehat{p}_{i_{u}}}} \widehat{\Phi}\left(\frac{\log \widehat{p}_{i_{u}}}{\log \left(q^{r}\right)}\right)\right)^{\varpi_{u}^{(\sigma)}} x\left(\varpi_{u}^{(\sigma)}, r, 0\right)
$$

and

$$
\mathrm{TE}:=\frac{1}{q \log ^{\alpha}\left(q^{r}\right)} \sum_{s=1}^{\alpha} \sum_{\sigma \in P(\alpha, s)} \sum_{\substack{i_{1}, \ldots, i_{s} \\ \text { distinct }}} \prod_{u=1}^{s}\left(\widehat{p}_{i_{u}}^{(r-2) / 4} \log ^{2} \widehat{p}_{i_{u}}\left|\widehat{\Phi}\left(\frac{\log \widehat{p}_{i_{u}}}{\log \left(q^{r}\right)}\right)\right|\right)^{\varpi_{u}^{(\sigma)}} .
$$

We have

$$
\mathrm{TE}=\frac{1}{q \log ^{\alpha}\left(q^{r}\right)}\left(\sum_{\substack{p \in \mathcal{P} \\ p \nmid q}} p^{(r-2) / 4} \log ^{2} p\left|\widehat{\Phi}\left(\frac{\log p}{\log \left(q^{r}\right)}\right)\right|\right)^{\alpha} \ll q^{\alpha r \nu(r+2) / 4-1}
$$

so that, TE is an error term as soon as

$$
\alpha r \nu(r+2)<4 \text {. }
$$

We assume from now on that this condition is satisfied. According to (2.14) (recall that $r \geqslant 1$ ), we rewrite $(6.21)$ as

$$
\mathrm{TP}=\frac{(-2)^{\alpha}}{\log ^{\alpha}\left(q^{r}\right)} \sum_{s=1}^{\alpha} \sum_{\sigma \in P \geqslant 2(\alpha, s)} \sum_{\begin{array}{c}
i_{1}, \ldots, i_{s} \\
\text { distinct }
\end{array}} \prod_{u=1}^{s}\left(\frac{\log \widehat{p}_{i_{u}}}{\sqrt{\widehat{p}_{i_{u}}}} \widehat{\Phi}\left(\frac{\log \widehat{p}_{i_{u}}}{\log \left(q^{r}\right)}\right)\right)^{\varpi_{u}^{(\sigma)}} x\left(\varpi_{u}^{(\sigma)}, r, 0\right)
$$

where

$$
P^{\geqslant 2}(\alpha, s):=\left\{\sigma \in P(\alpha, s): \forall u \in\{1, \ldots, s\}, \varpi_{u}^{(\sigma)} \geqslant 2\right\} .
$$


Moreover, if for at least one $\sigma$ and at least one $u$ (say $u_{0}$ ) we have $\varpi_{u}^{(\sigma)} \geqslant 3$, then

$$
\begin{aligned}
& \sum_{\substack{i_{1}, \ldots, i_{s} \\
\text { distinct }}} \prod_{u=1}^{s}\left(\frac{\log \widehat{p}_{i_{u}}}{\sqrt{\widehat{p}_{i_{u}}}} \widehat{\Phi}\left(\frac{\log \widehat{p}_{i_{u}}}{\log \left(q^{r}\right)}\right)\right)^{\varpi_{u}^{(\sigma)}} x\left(\varpi_{u}^{(\sigma)}, r, 0\right) \\
& \ll\left(\sum_{\substack{p \in \mathcal{P} \\
p \leqslant q^{r \nu}}} \frac{\log ^{3}(p)}{p^{3 / 2}}\right) \prod_{\substack{u=1 \\
u \neq u_{0}}}^{s}\left(\sum_{\substack{p_{u} \in \mathcal{P} \\
p_{u} \leqslant q^{r \nu}}} \frac{\log ^{2}\left(p_{u}\right)}{p_{u}}\right) \\
& \ll(\log q)^{2 s-2} .
\end{aligned}
$$

But, from (6.6), we deduce

$$
2 s \leqslant \sum_{j=1}^{s} \varpi_{j}^{(\sigma)}=\alpha
$$

hence $(\log q)^{2 s-2} \ll(\log q)^{\alpha-2}$. Reinserting this in (6.26) and the result in (6.25), we obtain

$$
\begin{aligned}
& \mathrm{TP}=\frac{(-2)^{\alpha}}{\log ^{\alpha}\left(q^{r}\right)} \sum_{s=1}^{\alpha} \sum_{\sigma \in P^{2}(\alpha, s)} \sum_{\begin{array}{c}
i_{1}, \ldots, i_{s} \\
\text { distinct }
\end{array}} \prod_{u=1}^{s}\left(\frac{\log \widehat{p}_{i_{u}}}{\sqrt{\widehat{p}_{i_{u}}}} \widehat{\Phi}\left(\frac{\log \widehat{p}_{i_{u}}}{\log q^{r}}\right)\right)^{\varpi_{u}^{(\sigma)}} x\left(\varpi_{u}^{(\sigma)}, r, 0\right) \\
& +O\left(\frac{1}{\log ^{2}(q)}\right)
\end{aligned}
$$

where

$$
P^{2}(\alpha, s):=\left\{\sigma \in P(\alpha, s): \forall u \in\{1, \ldots, s\}, \varpi_{u}^{(\sigma)}=2\right\} .
$$

From (6.27), (6.23) and (6.20), we deduce

$$
\begin{array}{r}
\mathbb{E}_{q}^{\mathrm{h}}\left(P_{q}^{1}[\Phi ; r]^{\alpha}\right)=\frac{(-2)^{\alpha}}{\log ^{\alpha}\left(q^{r}\right)} \sum_{s=1}^{\alpha} \sum_{\sigma \in P^{2}(\alpha, s)} \sum_{\begin{array}{c}
i_{1}, \ldots, i_{s} \\
\text { distinct }
\end{array}} \prod_{u=1}^{s} \frac{\log ^{2}\left(\widehat{p}_{i_{u}}\right)}{\widehat{p}_{i_{u}}} \widehat{\Phi}^{2}\left(\frac{\log \widehat{p}_{i_{u}}}{\log \left(q^{r}\right)}\right) \\
+O\left(\frac{1}{\log ^{2}(q)}\right)
\end{array}
$$

since $x(2, r, 0)=1$ according to (2.14). Note in particular that, according to (6.6) the previous sum is zero if $\alpha$ is odd. Thus, we can assume now that $\alpha$ is even and get

$$
\begin{array}{r}
\mathbb{E}_{q}^{\mathrm{h}}\left(P_{q}^{1}[\Phi ; r]^{\alpha}\right)=\frac{(-2)^{\alpha}}{\log ^{\alpha}\left(q^{r}\right)} \sum_{\sigma \in P^{2}(\alpha, \alpha / 2)} \sum_{\begin{array}{c}
i_{1}, \ldots, i_{\alpha / 2} \\
\text { distinct }
\end{array}} \prod_{u=1}^{\alpha / 2} \frac{\log ^{2}\left(\widehat{p}_{i_{u}}\right)}{\widehat{p}_{i_{u}}} \widehat{\Phi}^{2}\left(\frac{\log \widehat{p}_{i_{u}}}{\log \left(q^{r}\right)}\right) \\
+O\left(\frac{1}{\log ^{2}(q)}\right) .
\end{array}
$$


However, summing over all the possible $\left(i_{1}, \ldots, i_{\alpha / 2}\right)$ instead of the one with distinct indices reintroduces convergent sums that enter the error term because of the $1 / \log ^{\alpha}\left(q^{r}\right)$ factor. It follows that (6.29) becomes:

$$
\begin{array}{r}
\mathbb{E}_{q}^{\mathrm{h}}\left(P_{q}^{1}[\Phi ; r]^{\alpha}\right)=\left[\frac{4}{\log ^{2}\left(q^{r}\right)} \sum_{p \in \mathcal{P}} \frac{\log ^{2}(p)}{p} \widehat{\Phi}^{2}\left(\frac{\log p}{\log \left(q^{r}\right)}\right)\right]^{\alpha / 2} \# P^{2}(\alpha, \alpha / 2) \\
+O\left(\frac{1}{\log ^{2}(q)}\right) \cdot
\end{array}
$$

Taking $m=2$ (we already proved that the second moment is finite, see section 5.1) and reinserting the result in (6.30) implies that

$$
\mathbb{E}_{q}^{\mathrm{h}}\left(P_{q}^{1}[\Phi ; r]^{\alpha}\right)=\mathbb{E}_{q}^{\mathrm{h}}\left(P_{q}^{1}[\Phi ; r]^{2}\right) \# P^{2}(\alpha, \alpha / 2)+O\left(\frac{1}{\log ^{2}(q)}\right) .
$$

We conclude by computing

$$
\# P^{2}(\alpha, \alpha / 2)=\frac{\alpha !}{2^{\alpha / 2}\left(\frac{\alpha}{2}\right) !} .
$$

(see [30, Example 5.2.6 and Exercise 5.43]).

6.4. Proof of the second bullet of proposition 6.1. We mix the two techniques which have been used to prove the first and third bullets of proposition 6.1. We get following the same lines and thanks to lemma 6.2

$$
\begin{aligned}
& \mathbb{E}_{q}^{\mathrm{h}}\left(P_{q}^{1}[\Phi ; r]^{m-\ell} P_{q}^{2}[\Phi ; r]^{\alpha}\right)=\frac{(-2)^{\alpha+m-\ell}}{\log ^{\alpha+m-\ell}\left(q^{r}\right)} \sum_{1 \leqslant j_{1}, \ldots, j_{\alpha} \leqslant r}(-1)^{\alpha r-\left(j_{1}+\ldots+j_{\alpha}\right)} \sum_{s=1}^{\alpha+m-\ell} \\
& \times \sum_{\sigma \in P(\alpha+m-\ell, s)} \sum_{\substack{i_{1}, \ldots, i_{s} \\
\text { distinct }}} \prod_{u=1}^{s}\left(\frac{\log \varpi_{u}^{(\sigma, 1)}+\varpi_{u}^{(\sigma, 2)}\left(\widehat{p}_{i_{u}}\right)}{\widehat{p}_{i_{u}}^{(\sigma, 1)} / 2+\varpi_{u}^{(\sigma, 2)}} \widehat{\Phi}\left(\frac{\log \widehat{p}_{i_{u}}}{\log \left(q^{r}\right)}\right)^{\varpi_{u}^{(\sigma, 1)}} \widehat{\Phi}\left(\frac{2 \log \widehat{p}_{i_{u}}}{\log \left(q^{r}\right)}\right)^{\varpi_{u}^{(\sigma, 2)}}\right) \\
& \times \mathbb{E}_{q}^{\mathrm{h}}\left(\prod_{u=1}^{s}\left(\lambda_{f}\left(\widehat{p}_{i_{u}}^{r}\right)^{\varpi_{u}^{(\sigma, 1)}} \prod_{j=1}^{r} \lambda_{f}\left(\widehat{p}_{i_{u}}^{2 j}\right)^{\varpi_{u, j}^{(\sigma, 2)}}\right)\right)
\end{aligned}
$$

where

$$
\begin{aligned}
& \varpi_{u}^{(\sigma, 1)}:=\#\{i \in\{1, \ldots, m-\ell\}, \sigma(i)=u\}, \\
& \varpi_{u}^{(\sigma, 2)}:=\#\{i \in\{1, \ldots, \alpha\}, \sigma(m-\ell+i)=u\}, \\
& \varpi_{u, j}^{(\sigma, 2)}:=\#\left\{i \in\{1, \ldots, \alpha\}, \sigma(m-\ell+i)=u \text { and } j_{i}=j\right\}
\end{aligned}
$$

for any $1 \leqslant u \leqslant s$, any $1 \leqslant j \leqslant r$ and any $\sigma \in P(\alpha+m-\ell, s)$. Note that these numbers satisfy

$$
\sum_{u=1}^{s}\left(\varpi_{u}^{(\sigma, 1)}+\varpi_{u}^{(\sigma, 2)}\right)=m-\ell+\alpha
$$

and

$$
\sum_{j=1}^{r} \varpi_{u, j}^{(\sigma, 2)}=\varpi_{u}^{(\sigma, 2)}
$$


for any $1 \leqslant u \leqslant r$ and any $\sigma \in P(\alpha+m-\ell, s)$ by definition. They also satisfy

$$
\forall \sigma \in P(\alpha+m-\ell, s), \forall u \in\{1, \ldots, s\}, \quad \varpi_{u}^{(\sigma, 1)}+\varpi_{u}^{(\sigma, 2)} \geqslant 1
$$

since any $\sigma \in P(\alpha+m-\ell, s)$ is surjective and

$$
\forall \sigma \in P(\alpha+m-\ell, s), \forall i \in\{1,2\}, \exists u_{i, \sigma} \in\{1, \ldots, s\}, \quad \varpi_{u_{i, \sigma}}^{(\sigma, i)} \geqslant 1
$$

since $\alpha \geqslant 1$ and $m-\ell \geqslant 1$. The strategy is to estimate individually each term of the $\sigma$-sum. Thus, we fix some integers $j_{1}, \ldots, j_{\alpha}$ in $\{1, \ldots, r\}$, some integer $s$ in $\{1, \ldots, r\}$ and some application $\sigma$ in $P(\alpha+m-\ell, s)$.

First case: $\quad \forall u \in\{1, \ldots, s\}, \varpi_{u}^{(\sigma, 1)} / 2+\varpi_{u}^{(\sigma, 2)} \leqslant 1$.

Let us remark that if $\varpi_{u}^{(\sigma, 2)}=1$ for some $1 \leqslant u \leqslant s$ then there exists a unique $1 \leqslant j_{i_{u}} \leqslant r$ depending on $\sigma$ such that $\varpi_{u, j_{i_{u}}}^{(\sigma, 2)}=1$ and $\varpi_{u, j}^{(\sigma, 2)}=0$ for any $1 \leqslant j \neq j_{i_{u}} \leqslant r$ according to (6.33). Thus,

$$
\begin{aligned}
& \prod_{u=1}^{s}\left(\lambda_{f}\left(\widehat{p}_{i_{u}}^{r}\right)^{\varpi_{u}^{(\sigma, 1)}} \prod_{j=1}^{r}\left(\lambda_{f}\left(\widehat{p}_{i_{u}}^{2 j}\right)^{\varpi_{u, j}^{(\sigma, 2)}}\right)\right)=\lambda_{f}\left(\prod_{\substack{1 \leqslant u \leqslant s \\
\left(\varpi_{u}^{(\sigma, 1)}, \varpi_{u}^{(\sigma, 2)}\right)=(2,0)}} \widehat{p}_{i_{u}}^{r \varpi_{u}^{(\sigma, 1)} / 2}\right) \\
& \times \lambda_{f}\left(\prod_{\substack{1 \leqslant u \leqslant s \\
\left(\varpi_{u}^{(\sigma, 1)}, \varpi_{u}^{(\sigma, 2)}\right)=(2,0)}} \widehat{p}_{i_{u}}^{r \varpi_{u}^{(\sigma, 1)} / 2} \prod_{\substack{1 \leqslant u \leqslant s \\
\left(\varpi_{u}^{(\sigma, 1)}, \varpi_{u}^{(\sigma, 2)}\right)=(1,0)}} \widehat{p}_{i_{u}}^{r \varpi_{u}^{(\sigma, 1)}} \prod_{\substack{1 \leqslant u \leqslant s \\
\left(\varpi_{u}^{(\sigma, 1)}, \varpi_{u}^{(\sigma, 2)}\right)=(0,1)}} \widehat{p}_{i_{u}}^{2 j_{i_{u}} \varpi_{u, j_{u}}^{(\sigma, 2)}}\right)
\end{aligned}
$$

where the two integers appearing in the right-hand side of the previous equality are different according to (6.35). Consequently, proposition 2.2 and lemmas 3.10 and 3.9 enable us to assert that

$$
\begin{gathered}
\mathbb{E}_{q}^{\mathrm{h}}\left(\prod_{u=1}^{s}\left(\lambda_{f}\left(\widehat{p}_{i_{u}}^{r}\right)^{\varpi_{u}^{(\sigma, 1)}} \prod_{j=1}^{r}\left(\lambda_{f}\left(\widehat{p}_{i_{u}}^{2 j}\right)^{\varpi_{u, j}^{(\sigma, 2)}}\right)\right)\right) \ll \frac{1}{q} \prod_{\substack{1 \leqslant u \leqslant s \\
\left(\varpi_{u}^{(\sigma, 1)}, \varpi_{u}^{(\sigma, 2)}\right)=(2,0)}} \frac{\log \widehat{p}_{i_{u}}}{\widehat{p}_{i_{u}}^{-r \varpi_{u}^{(\sigma, 1)} / 4}} \\
\times \prod_{\substack{1 \leqslant u \leqslant s \\
\left(\varpi_{u}^{(\sigma, 1)}, \varpi_{u}^{(\sigma, 2)}\right)=(1,0)}} \frac{\log \widehat{p}_{i_{u}}}{\widehat{p}_{i_{u}}^{-r \varpi_{u}^{(\sigma, 1)} / 4}} \prod_{\substack{1 \leqslant u \leqslant s \\
\left(\varpi_{u}^{(\sigma, 1)}, \varpi_{u}^{(\sigma, 2)}\right)=(0,1)}} \frac{\log \widehat{p}_{i_{u}}}{\widehat{p}_{i_{u}}^{-r \varpi_{u}^{(\sigma, 2)} / 2}} .
\end{gathered}
$$

Note that, in this first case, the right hand term is

$$
\frac{1}{q} \prod_{u=1}^{s} \frac{\log \widehat{p}_{i_{u}}}{\widehat{p}_{i_{u}}^{-r\left(\varpi_{u}^{(\sigma, 1)} / 4+\varpi_{u}^{(\sigma, 2)} / 2\right)}}
$$

hence the contribution of these $\sigma$ 's to $\mathbb{E}_{q}^{\mathrm{h}}\left(P_{q}^{1}[\Phi ; r]^{m-\ell} P_{q}^{2}[\Phi ; r]^{\alpha}\right)$ is bounded by

$$
\frac{q^{\varepsilon}}{q}\left(\sum_{p \leqslant q^{\nu r}} \frac{1}{p^{1 / 2-r / 4}}\right)^{m-\ell}\left(\sum_{p \leqslant q^{\nu r / 2}} \frac{1}{p^{1-r / 2}}\right)^{\alpha} \ll q^{\nu r / 4[(m-\ell)(r+2)+\alpha r]-1+\varepsilon} .
$$

This is an admissible error term as long as $\nu r / 4[(m-\ell)(r+2)+\alpha r]<1$. 
Second case: $\quad \exists u_{\sigma} \in\{1, \ldots, s\}, \varpi_{u_{\sigma}}^{(\sigma, 1)} / 2+\varpi_{u_{\sigma}}^{(\sigma, 2)}>1$.

According to (2.11) and (2.12), if $1 \leqslant u \leqslant s$ and $1 \leqslant j \leqslant r$ then

$$
\lambda_{f}\left(\widehat{p}_{i_{u}}^{r}\right)^{\varpi_{u}^{(\sigma, 1)}}=\sum_{k_{u, 1}=0}^{r \varpi_{u}^{(\sigma, 1)}} x\left(\varpi_{u}^{(\sigma, 1)}, r, k_{u, 1}\right) \lambda_{f}\left(\widehat{p}_{i_{u}}^{k_{u, 1}}\right)
$$

and

$$
\lambda_{f}\left(\widehat{p}_{i_{u}}^{2 j}\right)^{\varpi_{u, j}^{(\sigma, 2)}}=\sum_{k_{u, j, 2}=0}^{j \varpi_{u, j}^{(\sigma, 2)}} x\left(\varpi_{u, j}^{(\sigma, 2)}, 2 j, 2 k_{u, j, 2}\right) \lambda_{f}\left(\widehat{p}_{i_{u}}^{2 k_{u, j, 2}}\right)
$$

since $x\left(\varpi_{u, j}^{(\sigma, 2)}, 2 j, k_{u, j, 2}\right)=0$ if $k_{u, j, 2}$ is odd (see (2.14)). Then, one may remark that

$$
\prod_{1 \leqslant j \leqslant r} \lambda_{f}\left(\hat{p}_{i_{u}}^{2 k_{u, j, 2}}\right)=\sum_{\ell_{u}=0}^{K_{u}} y_{\ell_{u}} \lambda_{f}\left(\hat{p}_{i_{u}}^{2 \ell_{u}}\right)
$$

for some integers $y_{\ell_{u}}$ and where $K_{u}:=\sum_{1 \leqslant j \leqslant r} k_{u, j, 2}$ for any $1 \leqslant u \leqslant s$. All these facts lead to

$$
\begin{aligned}
& \mathbb{E}_{q}^{\mathrm{h}}\left(P_{q}^{1}[\Phi ; r]^{m-\ell} P_{q}^{2}[\Phi ; r]^{\alpha}\right)=\frac{(-2)^{\alpha+m-\ell}(-1)^{\alpha r}}{\log ^{\alpha+m-\ell}\left(q^{r}\right)} \sum_{1 \leqslant j_{1}, \ldots, j_{\alpha} \leqslant r}(-1)^{j_{1}+\ldots+j_{\alpha}} \sum_{s=1}^{\alpha+m-\ell} \\
& \times \sum_{\sigma \in P(\alpha+m-\ell, s)} \sum_{\substack{i_{1}, \ldots, i_{s} \\
\text { distinct }}} \prod_{u=1}^{s}\left(\frac{\log \varpi_{u}^{(\sigma, 1)}+\varpi_{u}^{(\sigma, 2)}\left(\widehat{p}_{i_{u}}\right)}{\widehat{p}_{i_{u}}^{(\sigma, 1)} / 2+\varpi_{u}^{(\sigma, 2)}} \widehat{\Phi}\left(\frac{\log \widehat{p}_{i_{u}}}{\log \left(q^{r}\right)}\right)^{\varpi_{u}^{(\sigma, 1)}} \widehat{\Phi}\left(\frac{2 \log \widehat{p}_{i_{u}}}{\log \left(q^{r}\right)}\right)^{\varpi_{u}^{(\sigma, 2)}}\right) \\
& \times \sum_{0 \leqslant k_{1,1} \leqslant r \varpi_{1}^{(\sigma, 1)}} \sum_{0 \leqslant k_{1,1,2} \leqslant \varpi_{1,1}^{(\sigma, 2)}} \ldots \sum_{0 \leqslant k_{1, r, 2} \leqslant r \varpi_{1, r}^{(\sigma, 2)}} \sum_{0 \leqslant \ell_{1} \leqslant K_{1}} \\
& \begin{array}{cccc}
\vdots & \vdots & \vdots & \vdots \\
0 \leqslant k_{s, 1} \leqslant r \varpi_{s}^{(\sigma, 1)} & 0 \leqslant k_{s, 1,2} \leqslant \varpi_{s, 1}^{(\sigma, 2)} & 0 \leqslant k_{s, r, 2} \leqslant r \varpi_{s, r}^{(\sigma, 2)} & 0 \leqslant \ell_{s} \leqslant K_{s}
\end{array} \\
& \times \prod_{u=1}^{s}\left(x\left(\varpi_{u}^{(\sigma, 1)}, r, k_{u, 1}\right) y_{\ell_{u}} \prod_{j=1}^{r}\left(x\left(\varpi_{u, j}^{(\sigma, 2)}, 2 j, 2 k_{u, j, 2}\right)\right)\right) \\
& \times \mathbb{E}_{q}^{\mathrm{h}}\left(\lambda_{f}\left(\prod_{u=1}^{s} \widehat{p}_{i_{u}}^{k_{u, 1}}\right) \lambda_{f}\left(\prod_{u=1}^{s} \widehat{p}_{i_{u}}^{2 \ell_{u}}\right)\right) .
\end{aligned}
$$

Proposition 2.2 and lemmas 3.10 and 3.9 enable us to assert that

$\mathbb{E}_{q}^{\mathrm{h}}\left(\lambda_{f}\left(\prod_{u=1}^{s} \widehat{p}_{i_{u}}^{k_{u, 1}}\right) \lambda_{f}\left(\prod_{u=1}^{s} \widehat{p}_{i_{u}}^{2 \ell_{u}}\right)\right)=\prod_{u=1}^{s} \delta_{k_{u, 1}, 2 \ell_{u}}+O\left(\frac{1}{q} \prod_{u=1}^{s} \widehat{p}_{i_{u}, 1 / 4+\ell_{u} / 2}^{k_{u}} \log \widehat{p}_{i_{u}}\right)$

and we can write

$$
\mathbb{E}_{q}^{\mathrm{h}}\left(P_{q}^{1}[\Phi ; r]^{m-\ell} P_{q}^{2}[\Phi ; r]^{\alpha}\right)=\mathrm{TP}+O(\mathrm{TE})
$$


with

$$
\begin{aligned}
& \mathrm{TP}:=\frac{(-2)^{\alpha+m-\ell}(-1)^{\alpha r}}{\log ^{\alpha+m-\ell}\left(q^{r}\right)} \sum_{1 \leqslant j_{1}, \ldots, j_{\alpha} \leqslant r}(-1)^{j_{1}+\ldots+j_{\alpha}} \sum_{s=1}^{\alpha+m-\ell} \\
& \times \sum_{\sigma \in P(\alpha+m-\ell, s)} \sum_{i_{1}, \ldots, i_{s}} \prod_{u=1}^{s}\left(\frac{\log _{u}^{\varpi_{u}^{(\sigma, 1)}+\varpi_{u}^{(\sigma, 2)}}\left(\widehat{p}_{i_{u}}\right)}{\widehat{p}_{i_{u}}^{(\sigma, 1)} / 2+\varpi_{u}^{(\sigma, 2)}} \widehat{\Phi}\left(\frac{\log \widehat{p}_{i_{u}}}{\log \left(q^{r}\right)}\right)^{\varpi_{u}^{(\sigma, 1)}} \widehat{\Phi}\left(\frac{2 \log \widehat{p}_{i_{u}}}{\log \left(q^{r}\right)}\right)^{\varpi_{u}^{(\sigma, 2)}}\right) \\
& \times \sum_{0 \leqslant k_{1,1,2} \leqslant \varpi_{1,1}^{(\sigma, 2)} \quad} \sum_{0 \leqslant k_{1, r, 2} \leqslant r \varpi_{1, r}^{(\sigma, 2)} 0 \leqslant \ell_{1} \leqslant r \min \left(\varpi_{1}^{(\sigma, 1)} / 2, \varpi_{1}^{(\sigma, 2)}\right)} \sum_{0 \leqslant k_{s, 1,2} \leqslant \varpi_{s, 1}^{(\sigma, 2)}} \sum_{0 \leqslant k_{s, r, 2} \leqslant r \varpi_{s, r}^{(\sigma, 2)} 0 \leqslant \ell_{s} \leqslant r \min \left(\varpi_{s}^{(\sigma, 1)} / 2, \varpi_{s}^{(\sigma, 2)}\right)} \\
& \quad \times \prod_{s=1}^{s}\left(x\left(\varpi_{u}^{(\sigma, 1)}, r, 2 \ell_{u}\right) y_{\ell_{u}} \prod_{j=1}^{r}\left(x\left(\varpi_{u, j}^{(\sigma, 2)}, 2 j, 2 k_{u, j, 2}\right)\right)\right)
\end{aligned}
$$

and

$$
\begin{aligned}
& \mathrm{TE}:=\frac{1}{q \log ^{\alpha+m-\ell}\left(q^{r}\right)} \\
& \times \sum_{s=1}^{\alpha+m-\ell} \sum_{\sigma \in P(\alpha+m-\ell, s)} \sum_{\substack{i_{1}, \ldots, i_{s} \\
\text { distinct }}} \prod_{u=1}^{s} \log ^{\varpi_{u}^{(\sigma, 1)}+\varpi_{u}^{(\sigma, 2)}+1}\left(\widehat{p}_{i_{u}}\right) \widehat{p}_{i_{u}}^{(r / 2-1)\left(\varpi_{u}^{(\sigma, 1)} / 2+\varpi_{u}^{(\sigma, 2)}\right)} \\
& \times\left|\widehat{\Phi}\left(\frac{\log \widehat{p}_{i_{u}}}{\log \left(q^{r}\right)}\right)\right|^{\varpi_{u}^{(\sigma, 1)}}\left|\widehat{\Phi}\left(\frac{2 \log \widehat{p}_{i_{u}}}{\log \left(q^{r}\right)}\right)\right|^{\varpi_{u}^{(\sigma, 2)}}
\end{aligned}
$$

which is bounded by $O_{\varepsilon}\left(q^{(\alpha+m-\ell) \nu r^{2} / 4-1+\varepsilon}\right)$ for any $\varepsilon>0$ and is an admissible error term if $(\alpha+m-\ell) \nu<4 / r^{2}$. Estimating TP is possible since we can assume that $\sigma$ satisfies the following additional property. If $\varpi_{u}^{(\sigma, 2)}=0$ for some $1 \leqslant u \leqslant s$ then $\varpi_{u}^{(\sigma, 1)}>1$. Let us assume on the contrary that $\varpi_{u}^{(\sigma, 1)} \leqslant 1$ which entails $\varpi_{u}^{(\sigma, 1)}=1$ according to (6.34). Then,

$$
x\left(\varpi_{u}^{(\sigma, 1)}, r, 2 \ell_{u}\right)=x(1, r, 0)=0
$$

since $\ell_{u}=0$ and according to (2.14). Thus, the contribution of the $\sigma$ 's which do not satisfy this last property vanishes. As a consequence, the sum over 
the distinct $i_{1}, \ldots, i_{s}$ is bounded by

$$
\begin{aligned}
\sum_{\substack{i_{1}, \ldots, i_{s} \\
\text { distinct }}} \prod_{\substack{\left.1 \varpi_{u}^{(\sigma, 1)}, \varpi_{u}^{(\sigma, 2)}\right)=(2,0) \\
\times}}\left(\frac{\log ^{2}\left(\widehat{p}_{i_{u}}\right)}{\widehat{p}_{i_{u}}}\left|\widehat{\Phi}\left(\frac{\log \widehat{p}_{i_{u}}}{\log \left(q^{r}\right)}\right)\right|^{2}\right) \\
\times \prod_{\substack{1 \leqslant u \leqslant s \\
\left(\varpi_{u}^{(\sigma, 1)}, \varpi_{u}^{(\sigma, 2)}\right)=(0,1)}}\left(\frac{\log \left(\widehat{p}_{i_{u}}\right)}{\widehat{p}_{i_{u}}}\left|\widehat{\Phi}\left(\frac{2 \log \widehat{p}_{i_{u}}}{\log \left(q^{r}\right)}\right)\right|\right) \\
\times \prod_{\substack{1 \leqslant u \leqslant s \\
\varpi_{u}^{(\sigma, 1)} / 2+\varpi_{u}^{(\sigma, 2)}>1}}\left(\frac{\log \varpi_{u}^{(\sigma, 1)}+\varpi_{u}^{(\sigma, 2)}\left(\widehat{p}_{i_{u}}\right)}{\widehat{p}_{i_{u}}^{(\sigma, 1)} / 2+\varpi_{u}^{(\sigma, 2)}}\left|\widehat{\Phi}\left(\frac{\log \widehat{p}_{i_{u}}}{\log \left(q^{r}\right)}\right)\right|^{\varpi_{u}^{(\sigma, 1)}}\left|\widehat{\Phi}\left(\frac{2 \log \widehat{p}_{i_{u}}}{\log \left(q^{r}\right)}\right)\right|^{\varpi_{u}^{(\sigma, 2)}}\right)
\end{aligned}
$$

which is itself bounded by $O\left(\log ^{A_{\sigma}}(q)\right)$ where the exponent is given by

$$
\begin{aligned}
A_{\sigma} & :=2 \#\left\{1 \leqslant u \leqslant s, \varpi_{u}^{(\sigma, 2)}=0 \text { and } \varpi_{u}^{(\sigma, 1)} / 2+\varpi_{u}^{(\sigma, 2)} \leqslant 1\right\} \\
& +\#\left\{1 \leqslant u \leqslant s, \varpi_{u}^{(\sigma, 2)}=1 \text { and } \varpi_{u}^{(\sigma, 1)} / 2+\varpi_{u}^{(\sigma, 2)} \leqslant 1\right\}<m-\ell+\alpha .
\end{aligned}
$$

The last inequality follows from (see (6.32) and the additional property of $\sigma)$

$$
m-\ell+\alpha=A_{\sigma}+\sum_{\substack{1 \leqslant u \leqslant s \\ \varpi_{u}^{(\sigma, 1)} / 2+\varpi_{u}^{(\sigma, 2)}>1}}\left(\varpi_{u}^{(\sigma, 1)}+\varpi_{u}^{(\sigma, 2)}\right) .
$$

Thus, the contribution of the TP term of these $\sigma$ 's to $\mathbb{E}_{q}^{\mathrm{h}}\left(P_{q}^{1}[\Phi ; r]^{m-\ell} P_{q}^{2}[\Phi ; r]^{\alpha}\right)$ is bounded by $O\left(\log ^{-1}(q)\right)$.

\section{Appendix A. Analytic and Arithmetic toolbox}

A.1. On smooth dyadic partitions of unity. Let $\psi: \mathbb{R}_{+} \rightarrow \mathbb{R}$ be any smooth function satisfying

$$
\psi(x)= \begin{cases}0 & \text { if } 0 \leqslant x \leqslant 1 \\ 1 & \text { if } x>\sqrt{2}\end{cases}
$$

and $x^{j} \psi^{(j)}(x) \ll_{j} 1$ for any real number $x \geqslant 0$ and any integer $j \geqslant 0$. If $\rho: \mathbb{R}_{+} \rightarrow \mathbb{R}$ is the function defined by

$$
\rho(x):= \begin{cases}\psi(x) & \text { if } 0 \leqslant x \leqslant \sqrt{2}, \\ 1-\psi\left(\frac{x}{\sqrt{2}}\right) & \text { otherwise }\end{cases}
$$

then $\rho$ is a smooth function compactly supported in [1,2] satisfying

$$
x^{j} \rho^{(j)}(x) \ll_{j} 1 \quad \text { and } \quad \sum_{a \in \mathbb{Z}} \rho\left(\frac{x}{\sqrt{2}^{a}}\right)=1
$$

for any real number $x \geqslant 0$ and any integer $j \geqslant 0$.

If $F: \mathbb{R}_{+}^{n} \rightarrow \mathbb{R}$ is a function of $n \geqslant 1$ real variables then we can decompose it in

$$
F=\sum_{a_{1} \in \mathbb{Z}} \cdots \sum_{a_{n} \in \mathbb{Z}} F_{A_{1}, \cdots, A_{n}}
$$




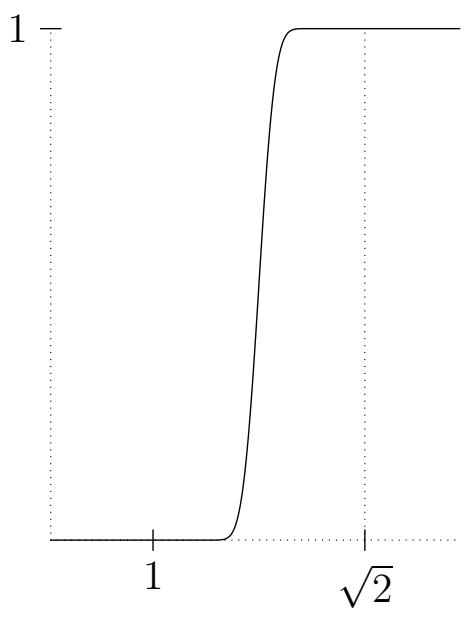

(a) Graph of $\psi$

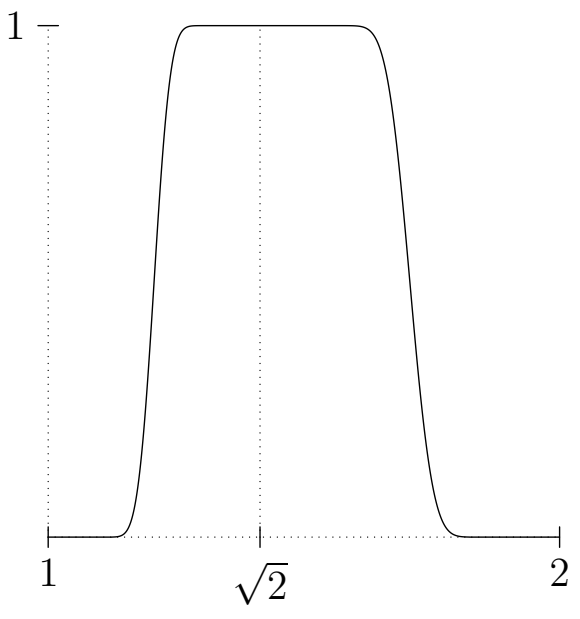

(b) Graph of $\rho$

where $A_{i}:=\sqrt{2}^{a_{i}}$ and

$$
F_{A_{1}, \cdots, A_{n}}\left(x_{1}, \cdots, x_{n}\right):=\prod_{i=1}^{n} \rho_{A_{i}}\left(x_{i}\right) F\left(x_{1}, \cdots, x_{n}\right)
$$

with $\rho_{A_{i}}\left(x_{i}\right):=\rho\left(x_{i} / A_{i}\right)$ is a smooth function compactly supported in $\left[A_{i}, 2 A_{i}\right]$ satisfying $x_{i}^{j} \rho_{A_{i}}^{(j)}\left(x_{i}\right) \ll_{j} 1$ for any real number $x_{i} \geqslant 0$ and any integer $j \geqslant 0$. Let us introduce the following notation for summation over powers of $\sqrt{2}$ :

$$
\sum_{A \leqslant M \leqslant B}^{\sharp} f(M):=\sum_{\substack{n \in \mathbb{N} \\ A \leqslant 2^{n / 2} \leqslant B}} f\left(2^{n / 2}\right) .
$$

We will use such smooth dyadic partitions of unity several times in this paper and we will also need these natural estimates in such contexts

$$
\sum_{M \leqslant M_{1}}^{\sharp} M^{\alpha} \ll M_{1}^{\alpha}
$$

for any $\alpha, M_{1}>0$ and

$$
\sum_{M \geqslant M_{0}}^{\sharp} \frac{1}{M^{\alpha}} \ll \frac{1}{M_{0}^{\alpha}}
$$

for any $\alpha, M_{0}>0$.

A.2. On Bessel functions. The Bessel function of first kind and order a integer $\kappa \geqslant 1$ is defined by

$$
\forall z \in \mathbb{C}, \quad J_{\kappa}(z):=\sum_{n \geqslant 0} \frac{(-1)^{n}}{n !(\kappa+n) !}\left(\frac{z}{2}\right)^{\kappa+2 n} .
$$

It satisfies the following estimate (founded in [23, Lemma C.2]), valid for any real number $x$, any integer $j \geqslant 0$ and any integer $\kappa \geqslant 1$ :

$$
\left(\frac{x}{1+x}\right)^{j} J_{\kappa}^{(j)}(x) \ll_{j, \kappa} \frac{1}{(1+x)^{\frac{1}{2}}}\left(\frac{x}{1+x}\right)^{\kappa}
$$


for any real number $x$, any integer $j \geqslant 0$ and any integer $\kappa \geqslant 1$. The following useful lemma follows immediately.

Lemma A.1- Let $X>0$ and $\kappa \geqslant 1$, then

$$
\sum_{d>0} \frac{\tau(d)}{\sqrt{d}}\left|J_{\kappa}\left(\frac{X}{d}\right)\right| \ll \begin{cases}X^{1 / 2} \log X & \text { if } X>1, \\ X^{\kappa} & \text { if } 0<X \leqslant 1 .\end{cases}
$$

A.3. Basic facts on Kloosterman sums. For any integer $m, n, c \geqslant 1$, the Kloosterman sum is defined by

$$
S(m, n ; c):=\sum_{x} e\left(\frac{m x+n \bar{x}}{c}\right)
$$

where $\bar{x}$ stands for the inverse of $x$ modulo $c$. We recall some basic facts on these sums. The Chinese remainder theorem implies the following multiplicativity relation

$$
S(m, n ; q r)=S\left(m \bar{q}^{2}, n ; r\right) S\left(m \bar{r}^{2}, n ; q\right)
$$

valid as soon as $(q, r)=1$. Here, $\bar{q}$ (resp. $\bar{r}$ ) is the inverse of $q$ (resp. $r$ ) modulo $r$ (resp. $q$ ). If $p$ and $q$ are two prime numbers, $\gamma \geqslant 1$ and $r \geqslant 1$ then, from (A.4) and $[7,(2.312)]$ we obtain

$$
S\left(p^{\gamma} q, 1 ; q r\right)= \begin{cases}-S\left(p^{\gamma} \bar{q}, 1 ; r\right) & \text { if }(q, r)=1, \\ 0 & \text { otherwise. }\end{cases}
$$

The Weil-Estermann inequality [6] is

$$
|S(m, n ; c)| \leqslant \sqrt{(m, n, c)} \tau(c) \sqrt{c} .
$$

\section{REFERENCES}

[1] A. O. L. Atkin and J. Lehner, Hecke operators on $\Gamma_{0}(m)$, Math. Ann. 185 (1970), 134-160. MR MR0268123 (42 \#3022)

[2] V. Blomer, G. Harcos, and P. Michel, A Burgess-like subconvex bound for twisted L-functions, Forum Math. 19 (2007), no. 1, 61-106.

[3] Joe P. Buhler, Benedict H. Gross, and Don B. Zagier, On the conjecture of Birch and Swinnerton-Dyer for an elliptic curve of rank 3, Math. Comp. 44 (1985), no. 170, 473-481. MR MR777279 (86g:11037)

[4] J. Cogdell and P. Michel, On the complex moments of symmetric power L-functions at $s=1$, Int. Math. Res. Not. (2004), no. 31, 1561-1617. MR MR2035301 (2005f:11094)

[5] J.-M. Deshouillers and H. Iwaniec, Kloosterman sums and Fourier coefficients of cusp forms, Invent. Math. 70 (1982/83), no. 2, 219-288. MR MR684172 (84m:10015)

[6] T. Estermann, On Kloosterman's sum, Mathematika 8 (1961), 83-86. MR MR0126420 (23 \#A3716)

[7] Th. Estermann, Vereinfachter Beweis eines Satzes von Kloosterman., Abhandlungen Hamburg 7 (1929), 82-98 (German).

[8] Stephen Gelbart and Hervé Jacquet, A relation between automorphic representations of GL(2) and GL(3), Ann. Sci. École Norm. Sup. (4) 11 (1978), no. 4, 471-542. MR MR533066 (81e:10025)

[9] Ahmet Muhtar Güloğlu, Low-lying zeroes of symmetric power L-functions, Int. Math. Res. Not. (2005), no. 9, 517-550. MR MR2131448

[10] E. Hecke, Über die Bestimmung Dirichletscher Reihen durch ihre Funktionalgleichung, Math. Ann. 112 (1936), no. 1, 664-699. MR MR1513069 
[11] _ Über Modulfunktionen und die Dirichletschen Reihen mit Eulerscher Produktentwicklung. I, Math. Ann. 114 (1937), no. 1, 1-28. MR MR1513122

[12] _ Über Modulfunktionen und die Dirichletschen Reihen mit Eulerscher Produktentwicklung. II, Math. Ann. 114 (1937), no. 1, 316-351. MR MR1513142

[13] C. P. Hughes, Mock-Gaussian behaviour, Recent perspectives in random matrix theory and number theory, London Math. Soc. Lecture Note Ser., vol. 322, Cambridge Univ. Press, Cambridge, 2005, pp. 337-355. MR MR2166468 (2006g:11191)

[14] C. P. Hughes and Z. Rudnick, Linear statistics of low-lying zeros of L-functions, Q. J. Math. 54 (2003), no. 3, 309-333. MR MR2013141 (2005a:11131)

[15] — Mock-Gaussian behaviour for linear statistics of classical compact groups, J. Phys. A 36 (2003), no. 12, 2919-2932, Random matrix theory. MR MR1986399 (2004e:60012)

[16] Henryk Iwaniec, Topics in classical automorphic forms, Graduate Studies in Mathematics, vol. 17, American Mathematical Society, Providence, RI, 1997. MR MR1474964 (98e:11051)

[17] Henryk Iwaniec and Emmanuel Kowalski, Analytic number theory, American Mathematical Society Colloquium Publications, vol. 53, American Mathematical Society, Providence, RI, 2004. MR MR2061214 (2005h:11005)

[18] Henryk Iwaniec, Wenzhi Luo, and Peter Sarnak, Low lying zeros of families of Lfunctions, Inst. Hautes Études Sci. Publ. Math. (2000), no. 91, 55-131 (2001). MR MR1828743 (2002h:11081)

[19] Nicholas M. Katz and Peter Sarnak, Random matrices, Frobenius eigenvalues, and monodromy, American Mathematical Society Colloquium Publications, vol. 45, American Mathematical Society, Providence, RI, 1999. MR MR1659828 (2000b:11070)

[20] Henry H. Kim, Functoriality for the exterior square of $\mathrm{GL}_{4}$ and the symmetric fourth of $\mathrm{GL}_{2}$, J. Amer. Math. Soc. 16 (2003), no. 1, 139-183 (electronic), With appendix 1 by Dinakar Ramakrishnan and appendix 2 by Kim and Peter Sarnak. MR MR1937203 (2003k:11083)

[21] Henry H. Kim and Freydoon Shahidi, Cuspidality of symmetric powers with applications, Duke Math. J. 112 (2002), no. 1, 177-197. MR MR1890650 (2003a:11057)

[22] - Functorial products for $\mathrm{GL}_{2} \times \mathrm{GL}_{3}$ and the symmetric cube for $\mathrm{GL}_{2}$, Ann. of Math. (2) 155 (2002), no. 3, 837-893, With an appendix by Colin J. Bushnell and Guy Henniart. MR MR1923967 (2003m:11075)

[23] E. Kowalski, P. Michel, and J. VanderKam, Rankin-Selberg L-functions in the level aspect, Duke Math. J. 114 (2002), no. 1, 123-191. MR MR1915038 (2004c:11070)

[24] Jean-François Mestre, Courbes de Weil et courbes supersingulières, Seminar on number theory, 1984-1985 (Talence, 1984/1985), Univ. Bordeaux I, Talence, 1985, pp. Exp. No. 23, 6. MR MR848380 (88b:11034)

[25] Stephen D. Miller, The highest lowest zero and other applications of positivity, Duke Math. J. 112 (2002), no. 1, 83-116. MR 1890648

[26] Steven J. Miller, One- and two-level densities for rational families of elliptic curves: evidence for the underlying group symmetries, Compos. Math. 140 (2004), no. 4, 952-992. MR MR2059225 (2005c:11085)

[27] Sami Omar, Majoration du premier zéro de la fonction zêta de Dedekind, Acta Arith. 95 (2000), no. 1, 61-65. MR MR1787205 (2001h:11143)

[28] Alexander Soshnikov, The central limit theorem for local linear statistics in classical compact groups and related combinatorial identities, Ann. Probab. 28 (2000), no. 3, 1353-1370. MR MR1797877 (2002f:15035)

[29] Richard P. Stanley, Enumerative combinatorics. Vol. 1, Cambridge Studies in Advanced Mathematics, vol. 49, Cambridge University Press, Cambridge, 1997, With a foreword by Gian-Carlo Rota, Corrected reprint of the 1986 original. MR MR1442260 (98a:05001)

[30] _ Enumerative combinatorics. Vol. 2, Cambridge Studies in Advanced Mathematics, vol. 62, Cambridge University Press, Cambridge, 1999, With a foreword by Gian-Carlo Rota and appendix 1 by Sergey Fomin. MR MR1676282 (2000k:05026) 
G. RICOTTA AND E. ROYER

\begin{tabular}{|c|c|c|}
\hline$\alpha_{2} \alpha_{1}$ & ] $0,1]$ & {$[1,+\infty[$} \\
\hline$] 0,1]$ & $\beta_{1}\left(1-\alpha_{1}\right)+\beta_{2}\left(1-\alpha_{2}\right)$ & $\beta_{2}\left(1-\alpha_{2}\right)$ \\
\hline$[1,+\infty[$ & $\beta_{1}\left(1-\alpha_{1}\right)$ & 0 \\
\hline
\end{tabular}

TABLE 1. Values of $\delta$

\begin{tabular}{|c|c|c|}
\hline$\varepsilon r$ & even & odd \\
\hline-1 & & $S O($ odd $)$ \\
\hline 1 & $S p$ & $S O($ even $)$ \\
\hline
\end{tabular}

